\title{
Measurements and Studies of Secondary Electron Emission of Diamond Amplified Photocathode
}

\author{
Qiong Wu
}

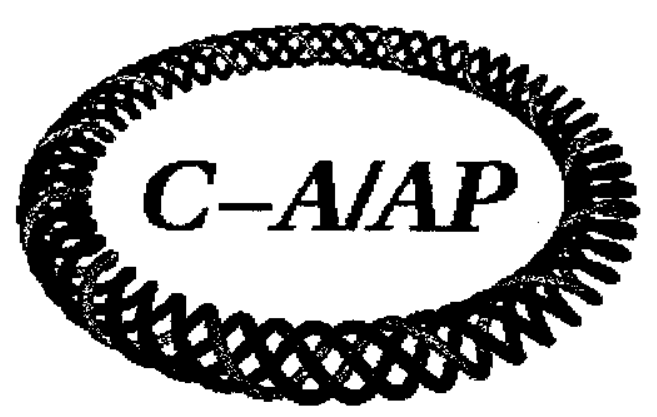

Collider-Accelerator Department Brookhaven National Laboratory

Upton, NY 11973

Notice: This document has been authorized by employees of Brookhaven Science Associates, LLC under Contract No. DE-AC02-98CH10886 with the U.S. Department of Energy. The United States Government retains a nonexclusive, paid-up, irrevocable, world-wide license to publish or reproduce the published form of this document, or allow others to do so, for United States Government purposes. 


\title{
MEASUREMENTS AND STUDIES OF SECONDARY ELECTRON EMISSION OF DiAMOND AMPLIFIED PHOTOCATHODE
}

\author{
Qiong $W u$
}

September, 2008 


\section{CONTENTS}

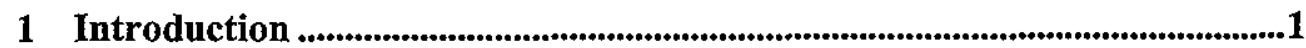

1.1 The Diamond Amplified Photocathode (DAP) Project.................................. 1

1.2 Advantages of diamond for amplified photocathodes .................................. 4

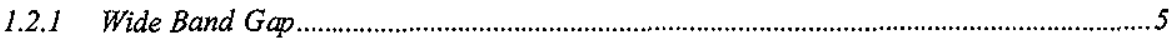

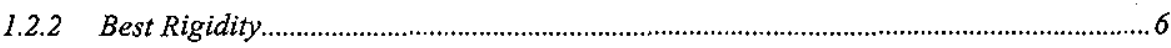

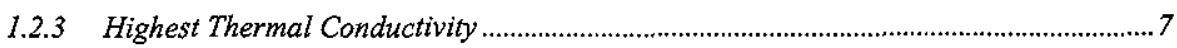

1.2.4 Very high Mobility and Saturation Velocity.............................................................

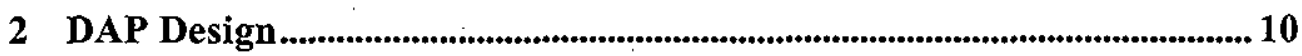

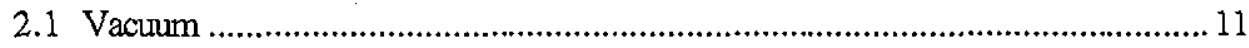

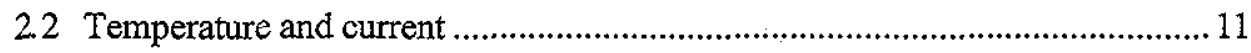

2.3 Energy bands and NEA surface in the diamond ......................................... 12

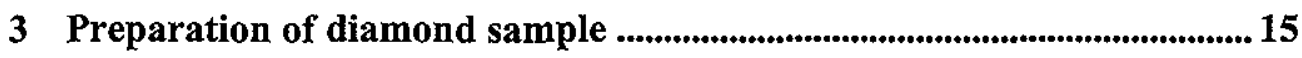

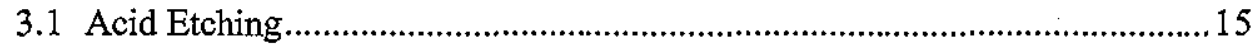

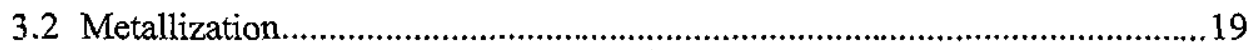

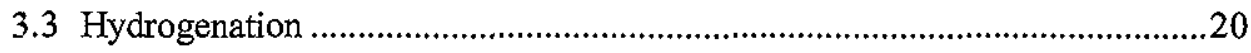


4 Diamond laser ablation ................................................................................ 22

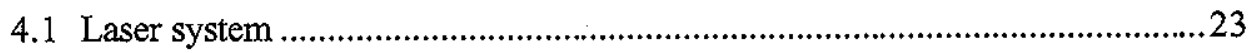

4.2 Sample preparation and characterization..................................................23

4.3 Laser ablation of polycrystalline diamond ...............................................2

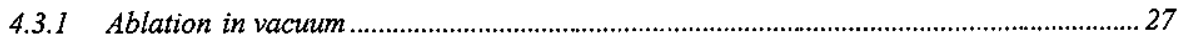

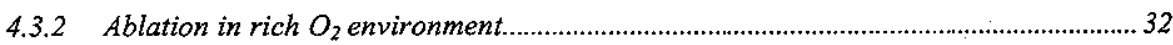

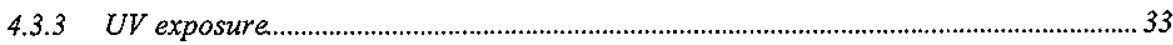

4.4 Laser ablation of single crystalline diamond..............................................35

5 Transmission mode measurement of Diamond Secondary electron yield

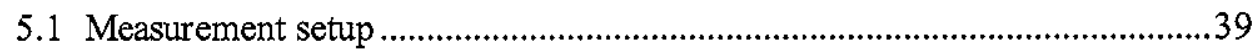

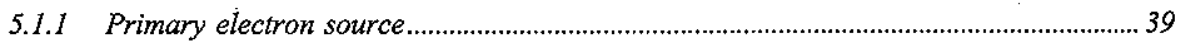

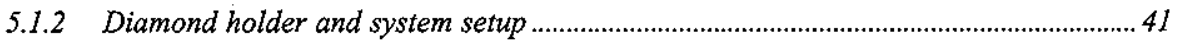

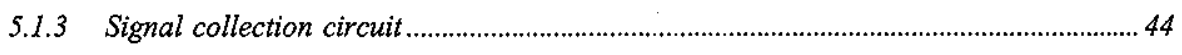

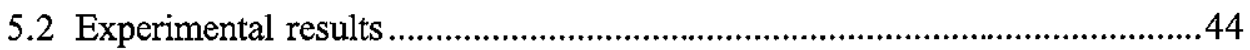

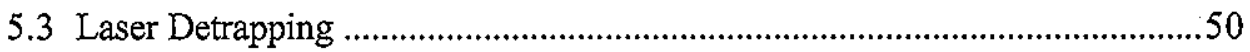




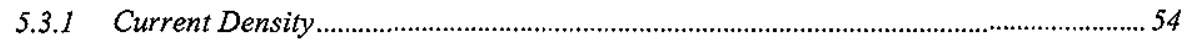

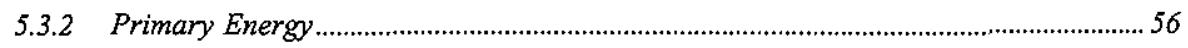

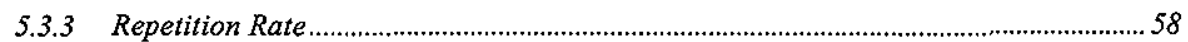

5.4 Energy lost in metal coating and estimation of energy needed for each $\mathrm{eh}$

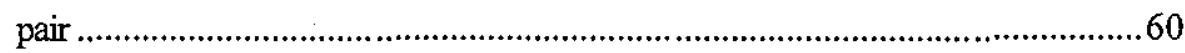

5.5 Recombination of the charges in diamond ...............................................62

\section{Emission Mode Measurement of Diamond Secondary Electron Yield. 67}

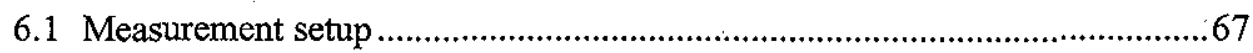

6.2 DC beam or continuous long pulse measurements......................................68

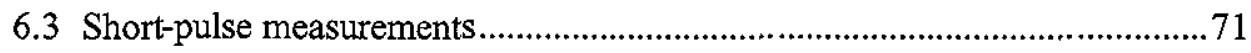

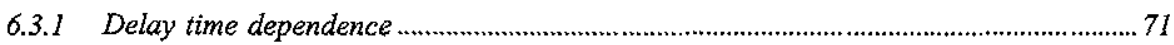

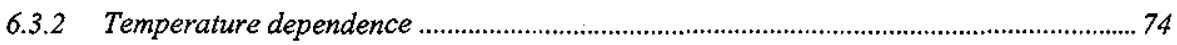

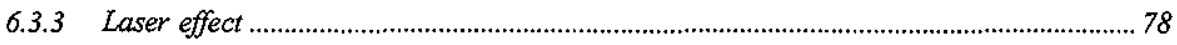

7 Thermal emittance measurement design ................................................ 82

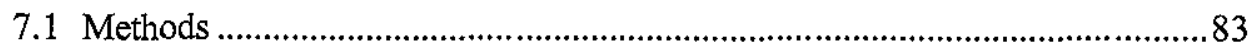




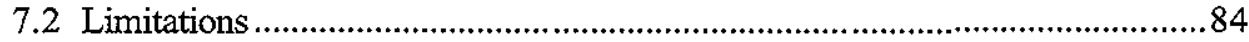

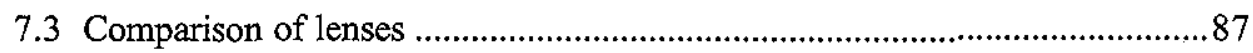

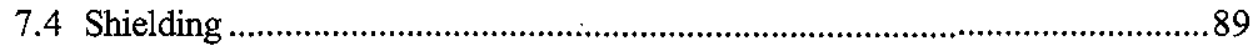

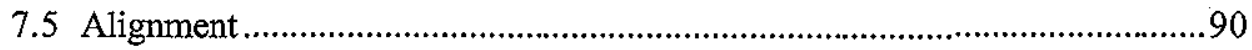

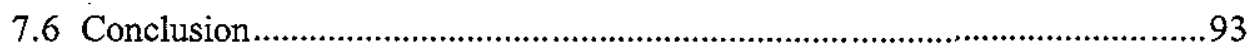

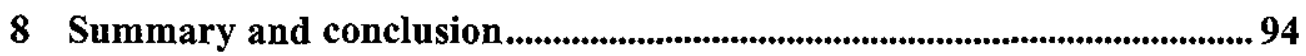




\section{INTRODUCTION}

\subsection{The Diamond Amplified Photocathode (DAP) Project}

Over many years, electron accelerators have served in a large variety of applications. Besides the contribution of electron colliders such as LEP in the precise measurements of important values in the Standard Model, scientists and engineers have made possible high brightness, constant power, and short wavelength light sources to be possible. Synchrotron Light Sources and Free Electron Lasers (FEL) provide the largest work platforms with high X-ray energy and brightness for biology, chemistry, material science, medical science, and much more. Many applications require continuous current, high intensity electron beams with narrow spatial and temporal distributions.

Traditionally electrons are produced in thermionic cathodes. Thermal cathodes rely on thermal energy overcoming the electrostatic forces restraining the charge carriers of certain materials. The emitted current is limited by the temperature of the cathode and the electric field. In recent years, photocathodes became widely used as an electron source. The negatively charged electrode covered with a layer of light sensitive material will emit electrons when struck by photons with energy above the material's band gap. This type of cathode can provide high brightness electron beams 
and low emittance when combined with the high electric field available in an RF gun.

Metallic materials such as magnesium and copper are popular in RF guns that operate with low average current $[1,2]$. These metallic materials are easy to prepare, robust and low in cost. The most important parameter for photocathodes is the quantum efficiency $(\mathrm{QE})$, which is the percentage of photons hitting the photoreactive material surface that will produce an e-h (electron-hole) pair. The $\mathrm{QE}$ of the metallic photocathodes is only in the order of $\sim 0.1 \%$. Semiconducting photocathodes, such as GaAs, can provide a much higher $\mathrm{QE}$ (in the order of $10 \%$ ) with a much larger photon energy range than metallic materials. But they are very prone to contamination so that the lifetime of semiconducting photocathodes is limited. Only a few hours of continuous emitting are axailable at the regime of about 100 milliamps, which is of interest for FEL and ERL [3]. The maximum current a photocathode can deliver depends upon the power of input laser and its QE, which is wavelength and material dependent.

The Diamond Amplified Photocathode (DAP) is a new development for electron sources that promise a high current, low emittance and high stability electron beam with a long lifetime. The DAP constitutes two main components, a traditional photocathode and an amplifier. The photocathode will provide a primary electron beam as a laser illuminates the surface material. These primary electrons will be accelerated to a few $\mathrm{keV}$ under a DC electric field applied between the photocathode and a thin metal layer coated on the amplifier window. The primary electrons will 
penetrate through the metal coating into the amplifier material and produce secondary electrons with amplification of more than 100 . The secondary electrons will be emitted into vacuum and accelerated by the RF field of the gun. Diamond is uniquely suited to be the amplifier's material as will be explained later on.

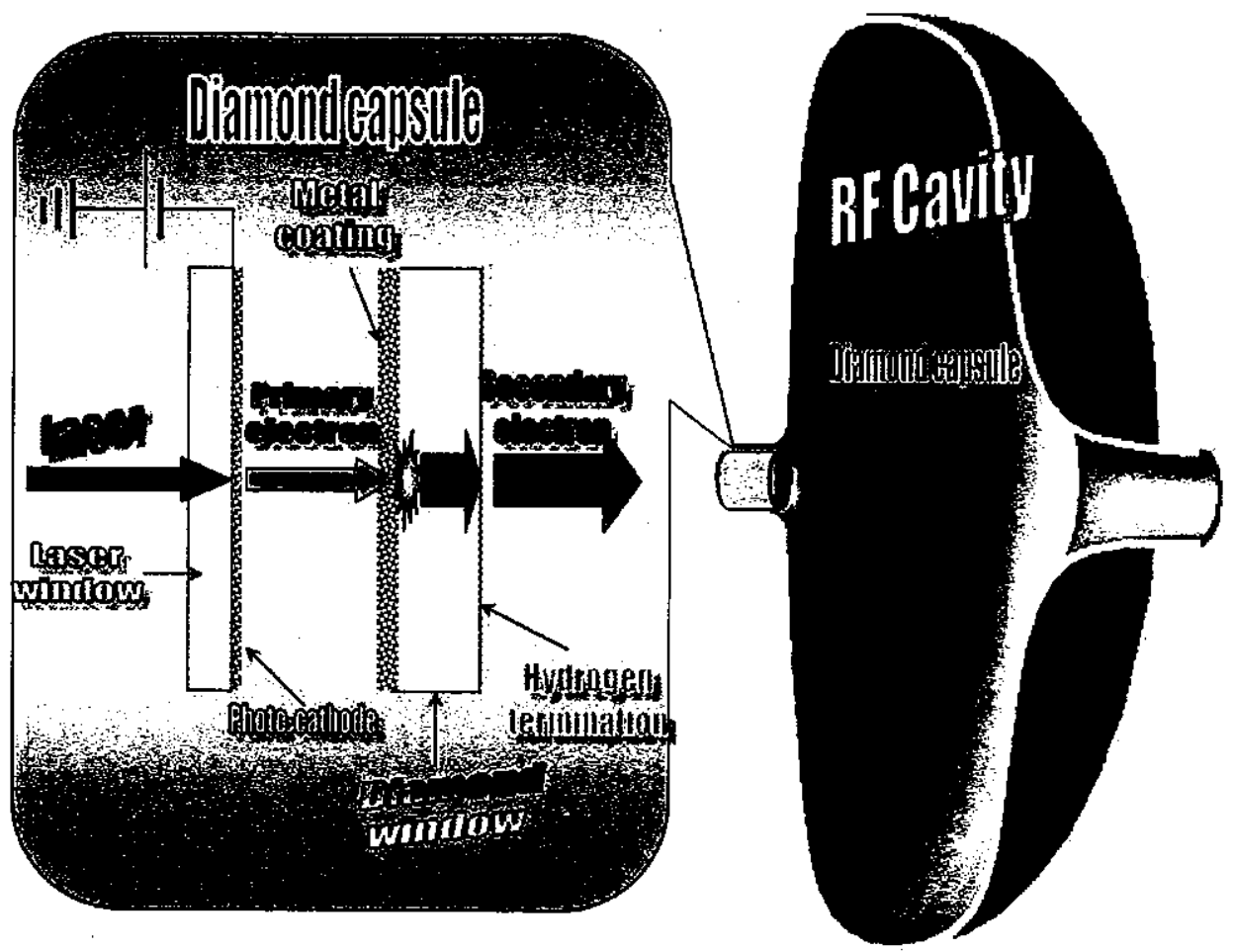

Figure 1.1: A complete DAP system.

Figure 1.1 shows the schematic drawing of the DAP system with diamond window as the amplifier. Diamond is ideal for this application, and its advantages will be discussed in the next section. 
The scenario inside the diamond window after primary electrons penetrate through the metal coating is as follows: Within about Donm travel in the diamond, the primary electrons generate secondary electrons and holes by collisions. The number of secondary electrons depends on the primary electron energy, but it is typically on the order of two orders of magnitudes larger than the number of primary electrons. Thus each primary electron will form a plasma cloud of electrons and holes along its range. The electron and holes will recombine within the plasma with the presence of three-body scattering or diffuse and merge into the metal coating to recombine within 1 ns unless separated by an electric field. If the RF field of the gun is carefully adjusted at the right phase to accelerate the electrons in the plasma cloud, portion of the secondary electrons will be able to transfer across the diamond bulk. The number of electrons that leave the plasma cloud is related to the RF electric field amplitude and the energy of primary electrons (will be discussed in Chapter 5). As the secondary electrons reach the opposite surface, they will exit into the vacuum through the diamond surface with hydrogen termination. The electrons emitted are "cold", i.e. they have a very low thermal emittance, for the electrons are constrained to the bottom of the conduction band.

\subsection{Advantages of diamond for amplified photocathodes}

The material of the amplifier is carefully selected to gain the highest Secondary Emission Yield (SEY). Among all the materials, diamond has many properties which 
allow it to provide the best performance as an amplifier of electron beams.

\subsubsection{Wide Band Gap}

Diamond is the prototype material for the structure shown in Figure 1.2. The conventional unit cell is face-centered cubic, with a side length $a_{0}$ approximately equal to $3.567 \AA(0.3567 \mathrm{~nm})$, and two atoms per unit cell at room temperature.

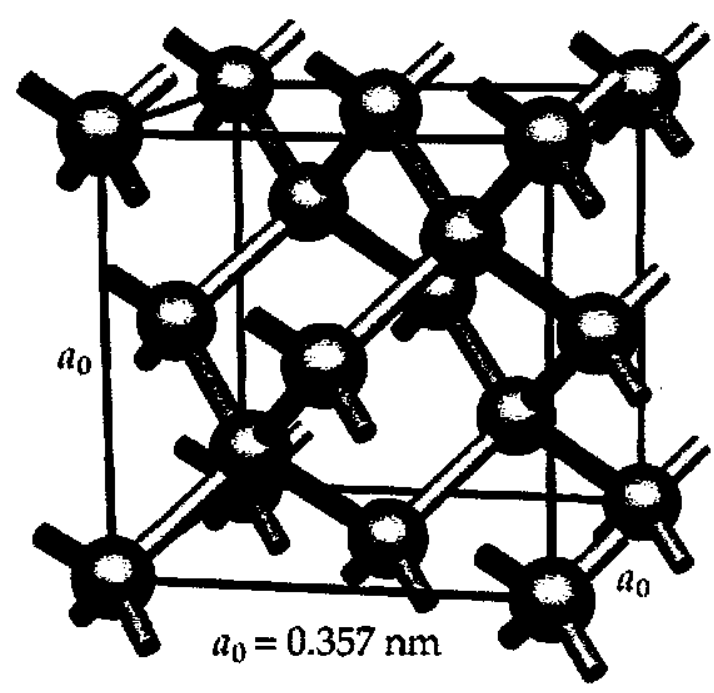

Figure 1.2: Diamond conventional unit cell.

Diamond has a band gap of $5.47 \mathrm{eV}$, which is very wide compared to other materials. Table 1.1 shows the minimum band gap energy of several common semiconductors. 
Table 1.1: Band gap energy list @300K[4].

\begin{tabular}{|c|c|c|}
\hline Material & Band Gap Energy $[\mathrm{eV}]$ & Breakdown field[MV/m] \\
\hline Diamond $(\mathrm{C})$ & 5.47 (indirect) & 2150 \\
\hline Silicon $(\mathrm{Si})$ & 1.11 (indirect) & 30 \\
\hline Silicon carbide $(\mathrm{SiC})$ & $2.36-3.23$ (indirect) & $100-500$ \\
\hline Germanium $(\mathrm{Ge})$ & 0.67 (indirect) & 0.1 \\
\hline Indium arsenide $(\mathrm{InAs})$ & 0.354 (direct) & 0.04 \\
\hline Gallium arsenide $(\mathrm{GaAs})$ & 1.43 (direct) & 60 \\
\hline Gallium nitride $(\mathrm{GaN})$ & 3.4 (direct) & 500 \\
\hline
\end{tabular}

Materiak with larger band gaps will have the ability of tolerant stronger electric fields. According to Table 1.1, diamond can hold up to more than $2000 \mathrm{MV} / \mathrm{m}$ electric field before breakdown. High electric field is required for preventing recombination and for increasing the drift velocity of the secondary electrons. As primary electrons create e-h pair plasma along their penetration path, the higher the applied field, the more secondary electrons can be pulled out before they diffuse into the metal coating.

Diamond is the only material that will have negative electron affinity (NEA) with hydrogen termination on the surface, due to the large band gap. This will be discussed further in Section 2.3.

\subsubsection{Best Rigidity}

It is well known that diamond is the most rigid material. This rigidity allows it to be prepared into thin films of only a few microns.

In principal, a thick diamond is desired for good thermal conductivity and 
strength. But for RF applications, the electron acceleration phase limit in the RF gun must be considered.

Calculations have been done by others for the single-cell RF gun designed for RHIC electron cooling. The optimized initial phase is less than $35^{\circ}$ and the frequency of RF cavity is $\sim 700 \mathrm{MHz}$. The RF phase for secondary electrons generated near the backside of the diamond should be at least $5^{\circ}$. The time allowed for secondary electrons to pass through the diamond is less than $30^{\circ}$, i.e. $\sim 120$ ps. The saturation velocity of electron transfer in diamond bulk is $\sim 2 \times 10^{5} \mathrm{~m} / \mathrm{s}$ [5]. Thus the thickness of the diamond window needs to be about 30 microns. For operation of a self-supporting film with a diameter of the order of $1 \mathrm{~cm}$ at this thickness the rigidity of diamond is a great advantage.

\subsubsection{Highest Thermal Conductivity}

The thermal conductivity of diamond is much higher than even copper, which is the most widely used thermal conducting material. The diamond is subjected to several heat sources during amplification of the current:

The power deposited by primary electrons bombardment.

The power deposited by secondary electron transportation.

The power deposited by RF shielding on the metal coating. 
The resistive heating by the electron replenishment current flowing through the metal coating.

The heat produced must be quickly dissipated to ensure the temperature of the whole system does not increase to an unsafe limit. Simulations in X. Chang's Ph.D. thesis showed that the diamond secondary emission cathode can easily handle $100 \mathrm{~W}$ of heat power without wide range temperature change [6].

Table 1.2: Thermal conduc tivity of various materials $[7,8]$.

\begin{tabular}{|c|c|c|}
\hline Material & Thermal conductivity $[\mathrm{W} /(\mathrm{m} \cdot \mathrm{K})]$ & Temperature $[\mathrm{K}]$ \\
\hline Diamond & 1000 & 273 \\
\hline Silver & 429 & 300 \\
\hline Copper & 386 & 279 \\
\hline Aluminum & 237 & 293 \\
\hline Stainless Steel & 16.3 & 296 \\
\hline Water & 0.6 & 293 \\
\hline Air & 0.025 & 293 \\
\hline
\end{tabular}

\subsubsection{Very high Mobility and Saturation Velocity}

The drift velocity of electrons in diamond is in the order of $1 \times 10^{5} \mathrm{~m} / \mathrm{s}$ under an electric field of a few megavolts per meter as mentioned above. High mobility is very important for high frequency applications. The electrons under high frequency RF field will only have a very small period of time to transfer through the amplifier in case to be at the right phase when emitted into the gun. Along with high rigidity, the 
diamond amplifier is easily capable of responding to $10 \mathrm{GHz}$ pulses. 


\section{DAP DESIGN}

The design of the DAP includes five main components designed to carry out the process of electron emission described in Chapter 1:

- Diamond amplifier with its metal coating

- Niobium substrate

- Sapphire vacuum cell

- Photocathode

- Supporting window

Figure 2.1 shows the relative size and configuration of the DAP. Individual issues of the DAP are discussed below.

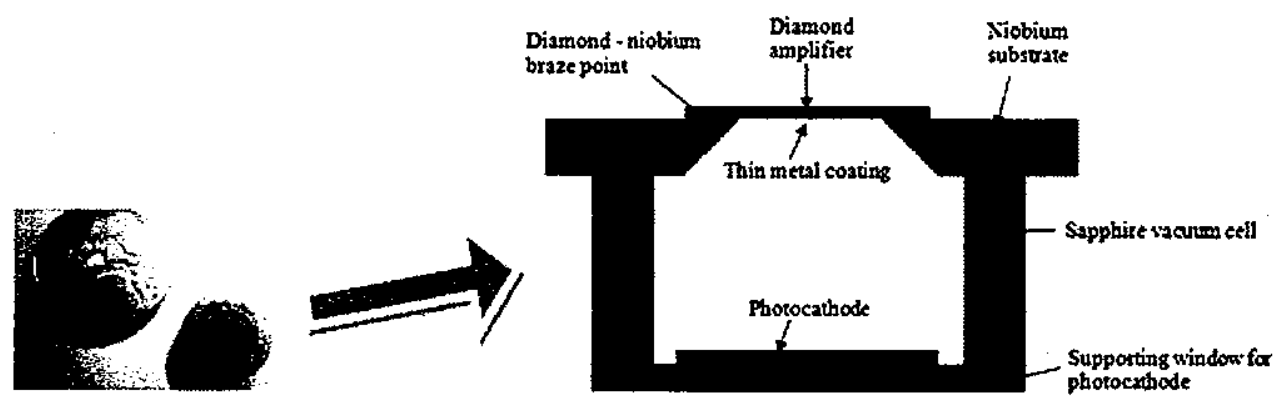

Figure 2.1:DAP Design (Photo on the left is taken by J. Grimes) 


\subsection{Vacuum}

Photocathode performance and lifetime can be very sensitive to certain gas density. An example is the cesium used in coating of a GaAs photocathode to provide Negative Electron Affinity (NEA). Cesium is very reactive and can be easily oxidized and lose its effect. The DAP capsule will protect the photocathode surface from contamination during transportation.

A $30 \mu \mathrm{m}$ thick diamond window with $10 \mathrm{~mm}$ diameter can hold up to $1 \mathrm{Atm}$ pressure difference. The vacuum inside the DAP can be maintained even during transfer of the assembly in air.

In addition to the diamond window rigidity, the brazing points must be vacuum tight [9].

\subsection{Temperature and current}

The selection of niobium and sapphire as amplifier substrate and supporting of the vacuum is based on their thermal expansion as well as their thermal and electrical conductivity.

The DAP is designed as an electron source for both normal and superconducting guns, thus it must be able to withstand repeated cryogenic cycle s to as low as a few degrees Kelvin. The preparation of the DAP involves diamond brazing, which is a high temperature process. Considering the above extreme thermal 
conditions, the material that is joined with the diamond needs to have a thermal expansion coefficient as close to diamond as possible to provide minimum stress. Good thermal conductivity is also important in the thermal processes.

As secondary electrons are emitted from the diamond, replenishment current is needed to keep the whol system neutral. Thus the joined material must also be electrical conductive.

Niobium with a thermal expansion coefficient of $7.3 \times 10^{-6} / \mathrm{K}$, electrical conductivity of $6.6 \times 10^{-6} \mathrm{O}^{-1} \mathrm{~m}^{-1}$, and thermal conductivity of $53.7 \mathrm{~W} /(\mathrm{m} \cdot \mathrm{K})$ is selected according to the above criteria [9].

Along the same line, the cell must be a high thermal conductive insulator to provide a good thermal path and electric standoff. Sapphire with thermal conductivity of $35 \mathrm{~W} /(\mathrm{m} \cdot \mathrm{K})$ at $300 \mathrm{~K}$, electrical conductivity of $1 \times 10^{-14} \mathrm{O}^{-1} \mathrm{~m}^{-1}$, and thermal expansion of $5.6 \times 10^{-6} / \mathrm{K}$ at $293 \mathrm{~K}$ is very suitable for these purposes.

2.3 Energy bands and NEA surface in the diamond

Diamond is a material that easily demonstrates NEA [10]. The electron affinity (EA) of an atom or molecule is the energy required to detach an electron from a singly charged negative ion, i.e. the energy change for the process. Most metallic atoms have more negative affinity than nonmetallic atoms. 
Table 2. 1: Electron affinity of carbon and hydrogen.

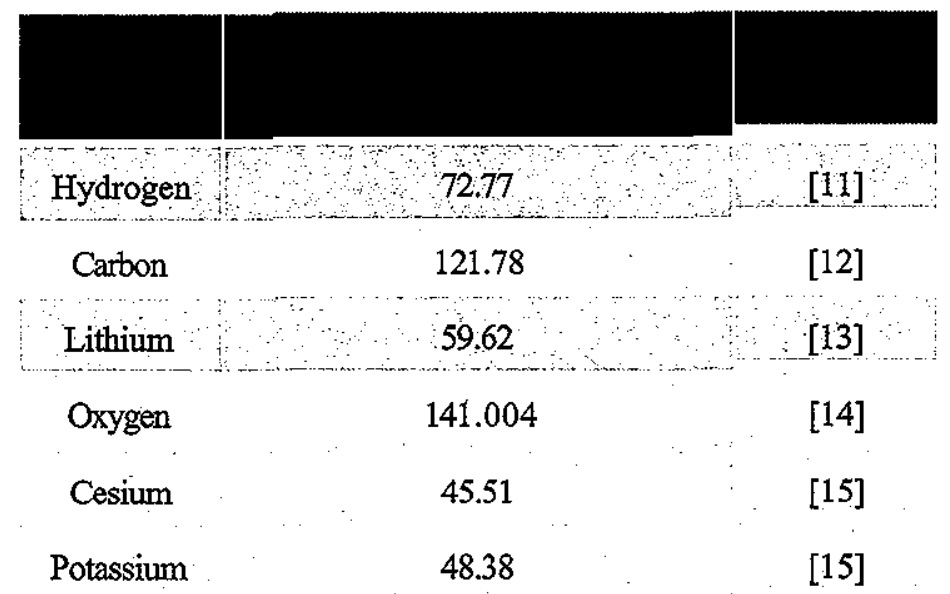

The EA of a surface is defined as the vacuum energy level $E_{\text {vac }}$ minus the conduction band minimum energy level $E_{c}$ :

$$
E A=E_{\text {vac }}-E_{c}
$$

The Fermi levels of the diamond and of the termination materials (hydrogen $\alpha$ alkaline elements) are aligned. Since the termination material has a relatively low work function, the vacuum level can be lower than the bottom level of the diamond's conduction band. This will allow the secondary electrons to escape into the vacuum.

As listed in Section 1.2.1, diamond has a very large band gap of $5.47 \mathrm{eV}$. As shown in Figure 2.2, the energy barrier from Fermi level to conduction band of diamond is higher than the work function of most of the metallic elements including hydrogen, which makes diamond very easy to form NEA. It is reported that the electron affinity of the hydr ogenated diamond surface can be as low as $-3.4 \mathrm{eV}[10,16$, 
17 .

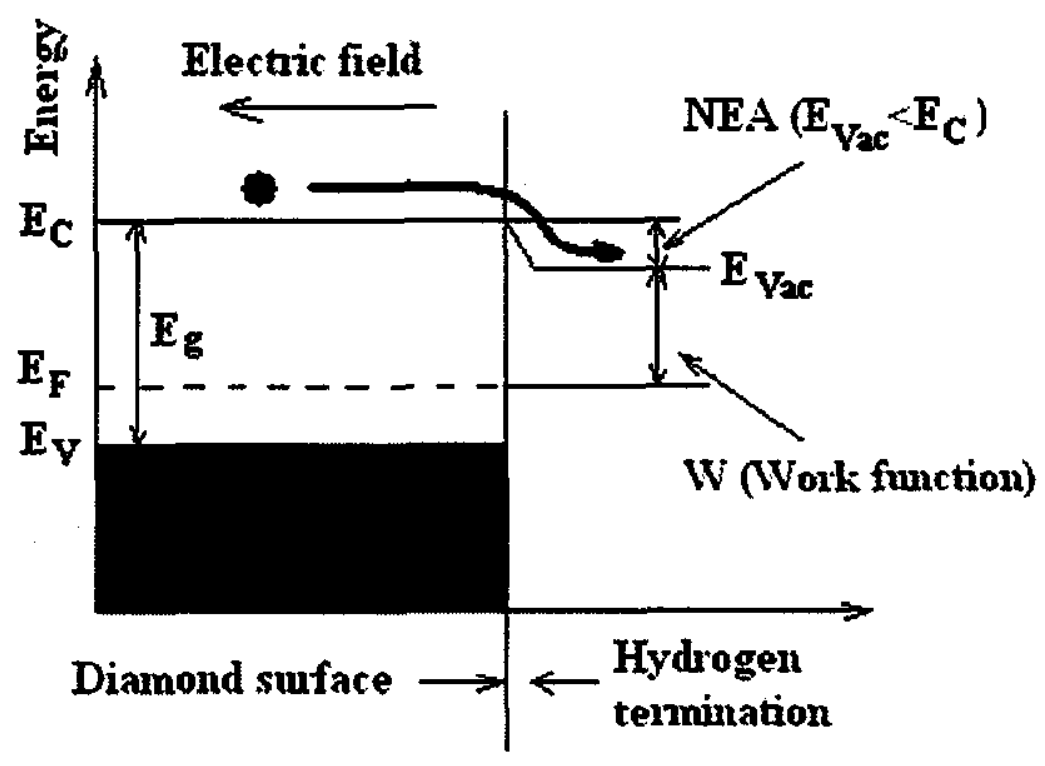

Figure 2.2: Field and energy bands in the diamond with NEA surface.

Photoemission and reflective mode secondary electron emission of hydrogenated diamond has been observed and reported [18-20]. For the application as current amplifier, secondary electrons need to transfer through the entire diamond bulk and only the non-recombined electrons can be emitted from the opposite surface. 


\section{PREPARATION OF DIAMOND SAMPLE}

\subsection{Acid Etching}

The purpose of acid etching is to eliminate the various atoms and non-diamond carbon layer present on the surface of the diamond sample. These elements and structures will define the Fermi level of the area they occupied, thus will add uncertainty to the experimental results.

\section{Procedure:}

- Prepare two work surfaces, one inside and another outside the fume hood. A hot plate should be placed inside the fume hood

- Prepare large glass containers that can be tightly closed for storing liquid waste

- Prepare a plastic bag to collect solid waste such as wipes

- Clean all the glassware to remove contaminants

- Mount the diamond samples in the sample holder

- Position two beakers with deionized water near the hot plate in the fume hood

- Wear Personal Protective Equipment (PPE) for safety.

- Ensure the ventilation system in the fume hood is on

- Place the chromic acid inside the fume hood. Chromic acid is hygroscopic, keep it tightly closed, away from moisture or any source of water 
- All the etching must be done under the fume hood. Keep hot liquids in the fume hood untill they are cool. After etching, make sure to dispose of the chemical waste in the pre-labeled glass containers. Follow the steps below:

- 15 min of saturated $\mathrm{CrO}_{5}$ in $\mathrm{H}_{2} \mathrm{SO}_{4}$ (heated so vapors are visible)

$\circ \quad$ Deionized (DI) water rinse

○ $2 \min \mathrm{NH}_{4} \mathrm{H}_{2} \mathrm{O} \quad(1: 10)$ Ultrasonic

- DI water rinse

- $2 \min \mathrm{HCl}: \mathrm{H}_{2} \mathrm{O} \quad(1: 10)$ Ultrasonic

- DI water rinse

- $5 \mathrm{Min}_{2} \mathrm{SO}_{4} \cdot \mathrm{H}_{2} \mathrm{O}_{2} \quad$ (5:1) boiling

- DI water rinse

- 2 min $\mathrm{NH}_{4}: \mathrm{H}_{2} \mathrm{O}_{2}: \mathrm{H}_{2} \mathrm{O}(1: 1: 4)$ boiling

- DI water rinse

- 2 min $\mathrm{HCl}: \mathrm{H}_{2} \mathrm{O}_{2}: \mathrm{H}_{2} \mathrm{O}(1: 1: 4)$ boiling

$\circ \quad$ DI water rinse

- DI water Ultrasonic

- DI water rinse 


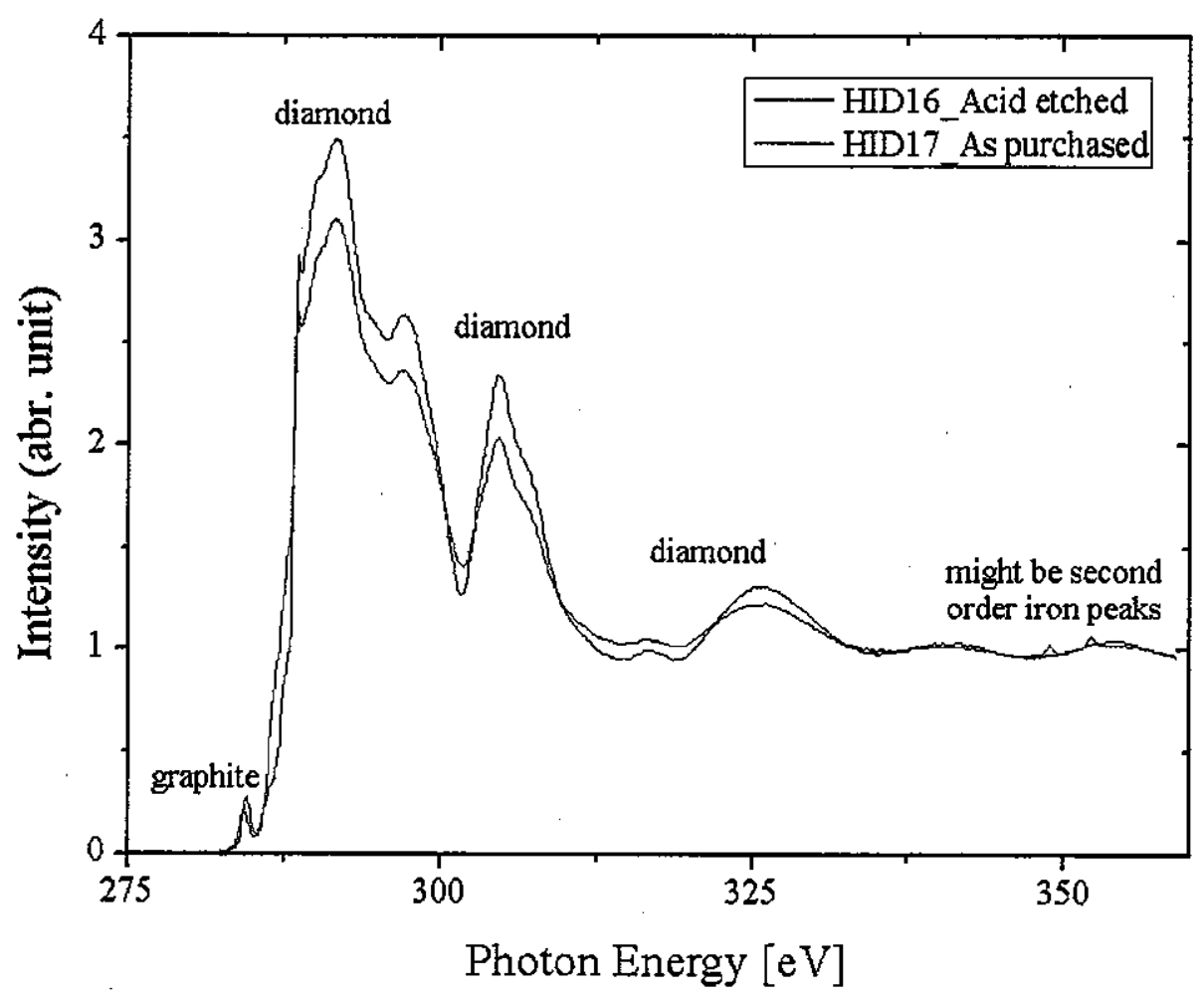

Figure 3.1: Near-edge $x$-ray absorption fine structure (NEXAF) comparison of diamond before and after acid cleaning.

In NEXAF, the photoelectron from core level excited by an input $\mathrm{x}$-ray photon is captured, and the consequent fluorescent photon or Auger electron or an inelastically scattered photoelectron may also be measured. All information is combined to determine all the final states of the photoelectron that are consistent with conservation rules. Detailed analysis of the NEXAFS data yields information about the orientation and the binding of the surface. The curves in Figure 3.1 have been normalized according to the photon flux, and the spectrum can be analyzed by comparing the 
peak locations to Ref [21]. Between the two normalized curves, the acid etched sample (blue curve) shows sharper diamond peaks and weaker non diamond peaks, which indicates the possibility for detecting a photoelectron from pure diamond bond is larger, i.e. the acid etching has eliminated the surface remnant for a certain degree.

The graphite peak around $280 \mathrm{eV}$ and second resonance peaks of iron around $350 \mathrm{eV}$ in Figure 3.1 gets smaller or vanishes completely after acid etching. The acid etching would partially remove the amorphous carbon and eliminates iron on the surface.

The strong acids used in the etching will create oxygen terminations on the diamond surface. The oxygen-carbon bond is very strong, with a bond energy $\mathrm{D}^{\circ}{ }_{298}(\mathrm{C}=\mathrm{O})=(1076.4 \pm 0.7) \mathrm{kJ} \cdot \mathrm{mol}^{-1}$ [22]. The oxygen termination will protect the purification of the surface from contamination until we heat it up to $800^{\circ} \mathrm{C}$ or more.

After acid etching, the sample is kept in a clean environment at $1 \%$ humidity for further experimentation Each sample is etched and stored separately for identification. We keep track of the history of each sample, so the metal coating migration into the diamond due to electron bombardment and lifetime of the sample can be evaluated.

The migration of metal coating layer into the diamond bulk has been observed on samples which has been used extensively, i.e. the thin metal coating on the surface cannot be removed by acid etching following extended electron bombardment. In this 
case, the diamond sample must be replaced.

\subsection{Metallization}

The signal collection highly depends on the details of the metallization of the primary side of the diamond sample. Primary electrons must penetrate the metal coating before entering the diamond for amplification. The metal coating will be in contact with the niobium substrate to provide a replenishment current to the diamond. All the electrons will lose part of their energy in the metal, and only the remnant energy will contribute in generating secondary electrons. Thus the metal coating has certain aspects that affect the performance of the amplification.

Clearly the selection of the coating material is important. The metal-diamond contact should be able to allow holes easily escape from the diamond, and strong adhesion to survive the extreme thermal process. The latter requirement prevents the usage of metals which do not interact with carbon once applied, such as gold. The former requirement therefore demands metals with EA close to that of the diamond to form an ohmic contact on the diamond surface. In fact, at this specific surface, the free carrier should be holes alone, which indicates that the surface will act as a p-type semiconductor, so metals with high work functions form the best contacts. In the experiments discussed in later chapters, the metal coating is a $\sim 50 \mathrm{~nm}$ platinum on top of $\sim 30 \mathrm{~nm}$ titanium, where the titanium forms a good bond with the diamond while the platinum provides protection of the titanium from oxidation. 


\subsection{Hydrogenation}

Hydrogenation is the process of applying hydrogen termination to the diamond surface. Before applying hydrogen atoms, the diamond surface must be freed from other elements by breaking their bonds at a high temperature, and usually this is done with local temperature of $800^{\circ} \mathrm{C}$ or higher. After exposing the bare diamond surface, the environment needs to be hydrogen atom rich. This can be done by flowing hydrogen gas through a hot filament or gas cracker, or applying a hydrogen plasma system. The hydrogenation in the experiments discussed in the following chapters was performed with pure hydrogen flowing through a thermal gas cracker (MANTIS MGC75) at $\sim 0.5$ cubic centimeters per minute.

\subsection{Brazing}

The configuration of the DAP as shown in Figure 2.1 can finished in two steps: brazing and cold welding.

The contacts between diamond and niobium, niobium and sapphire are produced by high temperature vacuum brazing with an alloy of titanium, copper, and silver (TiCuSil) as the brazing material. Ticusil is specially designed to join refractory metals and ceramic/diamond. This brazing technique is carried out by J. Grimes and described in detail in his MS thesis [9]. 
Cold welding is planned to be used to join the sapphire vacuum cell and the supporting window of the photocathode. Considering that the photocathode material is easily contaminated, it is important to keep it continuously under ultra-high vacuum. The best solution would be sealing the supporting window onto the sapphire in-situ at the preparation chamber of the photocathode. Cold welding is chosen to avoid a high temperature process that could ruin the performance of the photocathode. 


\section{DIAMOND LASER ABLATION}

The diamond thickness for amplifier application is critical, because together with the timing of the primary pulse, it defines the phase of the secondary emission pulse. In addition, a very smooth surface is required to prevent spreading the electrons in time, since the electron velocity is much slower in the diamond than in vacuum. For the same reason, a highly parallel geometry is required for the diamond plate. The ideal thickness to be used in a $700 \mathrm{MHz}$ electron gun for launch phase of $35^{\circ}$ would be about $30 \mu \mathrm{m}$. This is not achievable with mechanical polishing due to the stress embedded onto the surface during this process. Reactive Ion Etching (RIE) is one of the possible methods to obtain such a dimension, but this is a time consuming process if significant thickness reduction or large aspect ratios are required. The speed of the $\mathrm{RIE}$ thinning of the diamond that is commercially available is about $10 \mu \mathrm{m} /$ day.

As an alternative method, laser ablation was introduced for shaping the Chemical Vapor Deposition (CVD) diamonds. The advantages of this method, beyond thinning the diamond to tens of microns with 10 times faster speed than RIE, include the laser ablation can also engrave precise patterns onto the diamond surface or into the bulk as desired. 


\subsection{Laser system}

Two lasers, both operating at a fundamental wavelength of $1064 \mathrm{~nm}$ with pulse durations of $10 \mathrm{~ns}$ and $30 \mathrm{ps}$ were used for the ablation. The ps laser is an actively and passively mode locked Nd:YAG oscillator followed by pulse selector and double pass amplifier, (Leopard series, Continuum Lasers) operating at $1064 \mathrm{~nm}$, capable of delivering $125 \mathrm{~mJ}$ at $10 \mathrm{~Hz}$ in a pulse duration of $60 \mathrm{ps}$. The wavelength of this laser is converted to $266 \mathrm{~nm}$ by two successive doubling crystals to provide up to $13 \mathrm{~mJ}$ energy in $30 \mathrm{ps}$. Only the $266 \mathrm{~nm}$ radiation of this laser was used to ablate the sample. The energy on the target can be varied by changing the pump power of the amplifier.

The ns laser is a Q switched Nd:YAG capable of delivering up to $400 \mathrm{~mJ}$ in a 20 ns pulse duration at $10 \mathrm{~Hz}$ repetition rate. Two doubling crystals were used in series to generate up to $200 \mathrm{~mJ}$ of $532 \mathrm{~nm}$ and $40 \mathrm{~mJ}$ of $266 \mathrm{~nm}$ radiation with pulse durations of $14 \mathrm{~ns}$ and $10 \mathrm{~ns}$ respectively. The energy of the laser beam on the sample was adjusted by introducing appropriate filters in the beam path.

The beam from either of these lasers is focused using a nominal $15 \mathrm{~cm}$ lens to result in a $150 \mu \mathrm{m}$ focal spot size on the target. The position of the sample at the focus was identified by the size of the laser beam back reflected from the sample.

\subsection{Sample preparation and characterization}

The diamonds used in experiments are all single crystal, ultra-high-purity, highly 
polished and flat thin films produced by CVD. The size of the diamond samples used in the experiments is typically $4.0 \mathrm{~mm} \times 4.0 \mathrm{~mm} \times 0.3 \mathrm{~mm}$, and the surface orientation is [100]. Due to growth rate limitation, the other two orientations, [110] and [111] are not available in $\mathrm{ppb}$ purity level. The impurity concentration in these diamond samples are all within a few ppb. Both sides are polished to surface roughness ( $\mathrm{Ra})$ $<15 \mathrm{~nm}$, and the flatness is controlled to under 5 fringes @ $632.8 \mathrm{~nm}$ over the entire sample.

Atomic Force Microscope (AFM) scanning results of the diamond surface is shown in Figure 4.1.

High purity and small thickness single crystal diamonds contain few electron trapping centers. Impurity atoms, grain boundaries, and stress existing due to imperfect lattice structure are possible potential wells for electrons. Secondary electrons have a certain possibility being trapped by these potential wells. Electric field shielding is produced due to these trappings, which are discussed in $\operatorname{Ref}[6]$. 

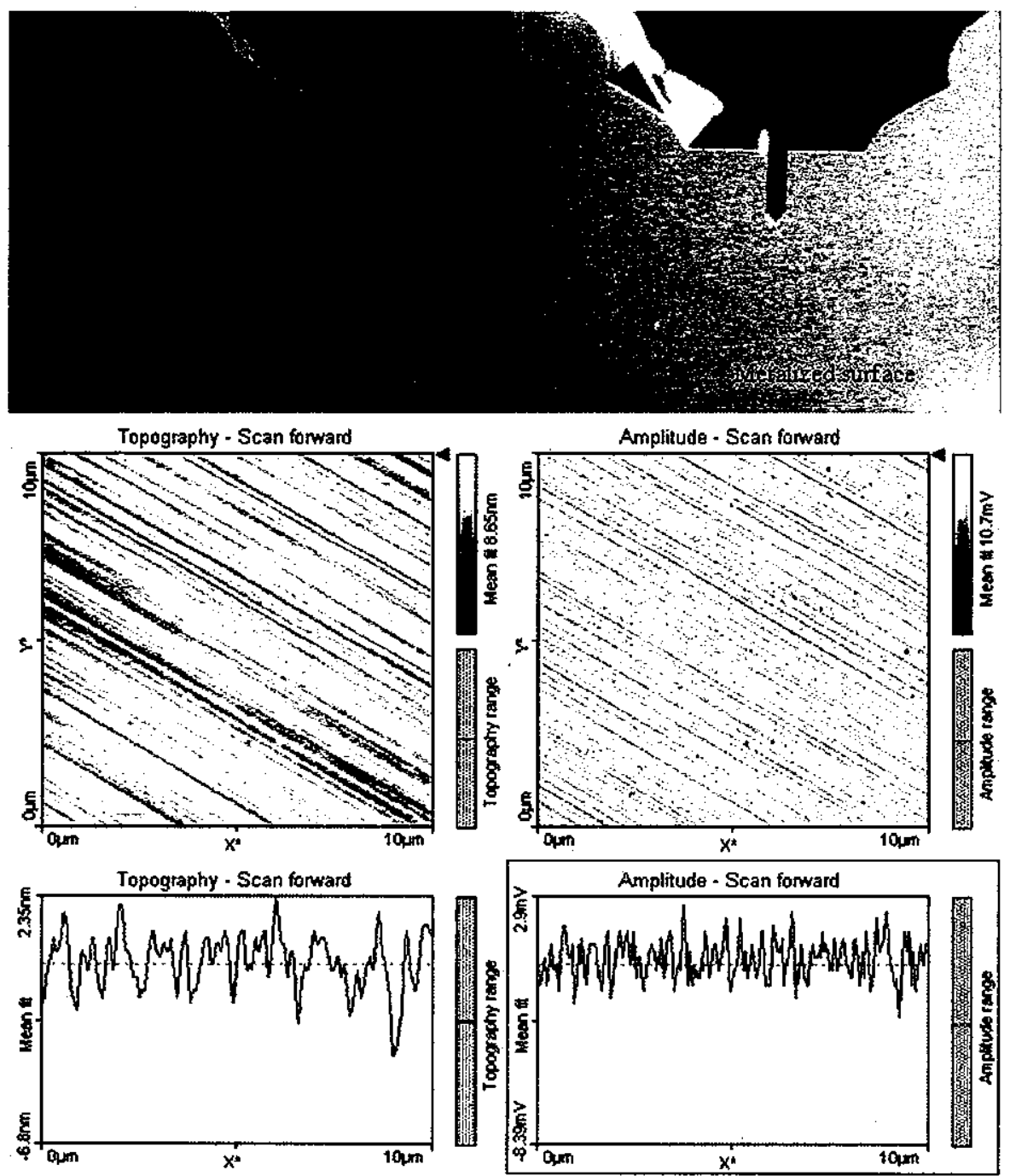

Figure 4.1: AFM scanning pictures of typical single crystal diamond sample bare and

metalized surfaces. Top two are taken by optical camera, and the structure on the bare diamond surface is the image of the holder of the diamond. The scanning cantilever width of the AFM is $0.04 \mathrm{~mm}$. The middle and bottom pictures are AFM scanning topography and feedback circuit signal of the bare diamond surface. Sample number

HID8. Sample scanned after ultrasonic cleaning in ethyl alcohol. 


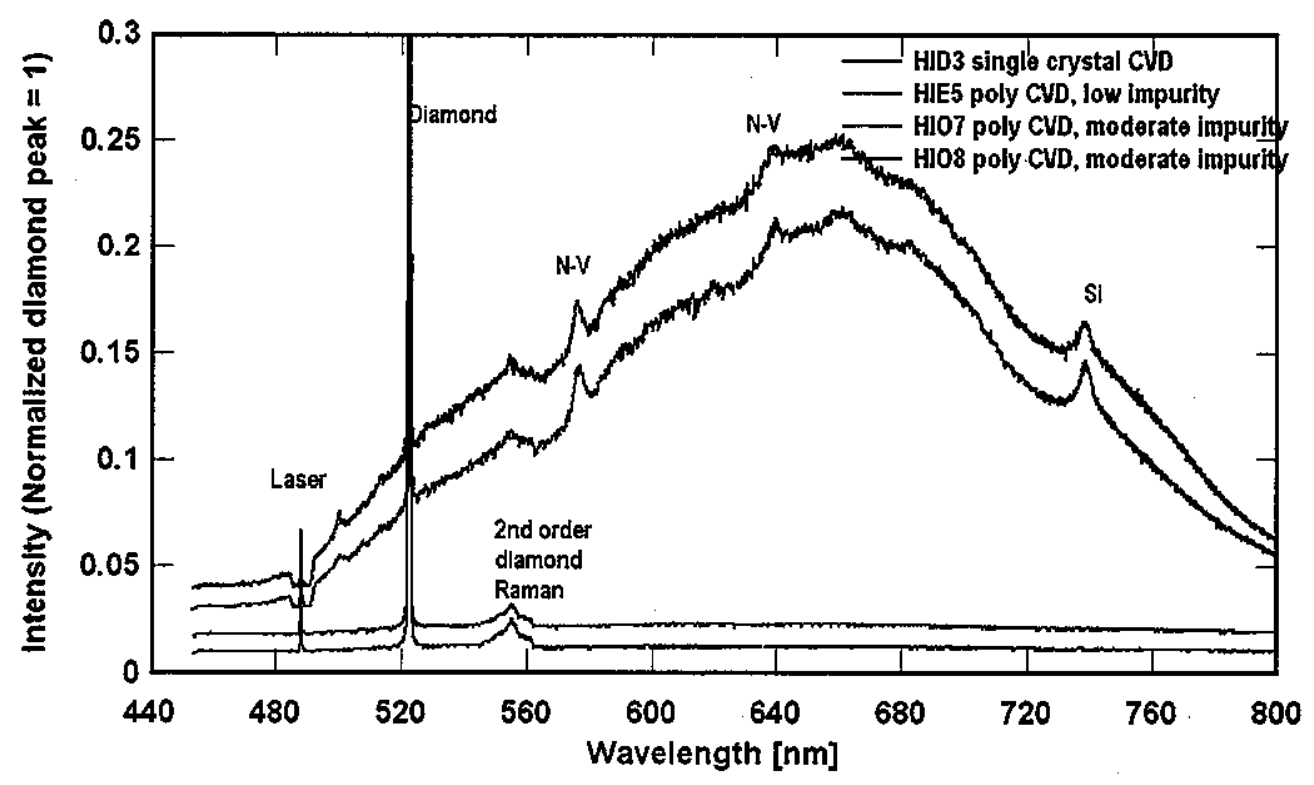

Figure 4.2: Raman/photoluminescence spectra of different diamond sample lattice.

In Raman/photoluminescence spectra above, electronic grade polycrystalline

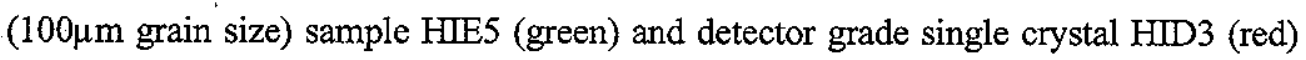
show negligible nitrogen impurities $(<1 \mathrm{ppm})$, while in optical grade polycrystalline

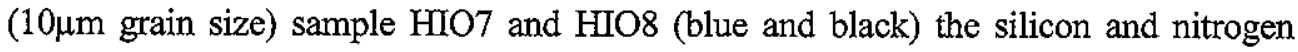
contents are significant. The optical grade samples also show large photoluminescence background, which is likely due to the abundant randomly oriented crystalline grain boundaries. In the secondary emission measurements, only detector grade samples are selected. This simplifies the motion of electrons drifting through the diamond, reduces the probability of trapping, and provides the best rigidity and thermal conductivity. 
Polycrystalline (electronic grade) and single crystal (detector grade) CVD diamonds from Harris International were used for laser ablation.

In other samples, these baseline measurements were done on unablated regions on the ablated samples. The sample is then mounted onto a holder that is suspended in a vacuum commercial $23 / 4$ inch vacuum cube held at pressures $<1 \mu$ torr. The cube is mounted on a dual axis, motorized translation stage to facilitate movement of the sample in a plane perpendicular to the laser beam and perform raster scanning. We characterized all the ablated samples using optical and atomic force microscopes, Fourier Transform Infrared Spectroscopy (FTIR) and Raman spectroscopy, Near Edge X-ray Absorption Fine Structure (NEXAFS) and x-ray crystallography where appropriate.

\subsection{Laser ablation of polycrystalline diamond}

\subsubsection{Ablation in vacuum}

Two identical electronic grade diamonds, labeled as HIE6 and HIE8, with dimensions of $5 \mathrm{~mm}$ diameter, $0.16 \mathrm{~mm}$ thick and optical finish were exposed in succession to $132 \mu \mathrm{J}$ of $532 \mathrm{~nm}$ and $90 \mu \mathrm{J}$ of $266 \mathrm{~nm}$ at $10 \mathrm{~Hz}$ repetition rate, and the pulse duration was tuned to be at $10 \mathrm{~ns}$ or $30 \mathrm{ps}$ for both wavelengths. The sample was pumped down as described above to better than $1 \mu$ Torr. The samples were moved in a raster pattern in both $\mathrm{x}$ and $\mathrm{y}$ directions 10 times with a scan step of $50 \mu \mathrm{m}$ for 70 minutes to ablate $1 \times 1 \mathrm{~mm}^{2}$ on the sample. Since these two samples were identical, in 
some cases, data from one sample before processing and from another after processing are compared to study the effect of the process.

The ablation with $532 \mathrm{~nm} \sim 10 \mathrm{~ns}$ pulsed laser was quite destructive, non-uniformly removing large regions of the diamond surface, although some raster scan pattern is observable, as shown in Figure 4.3.

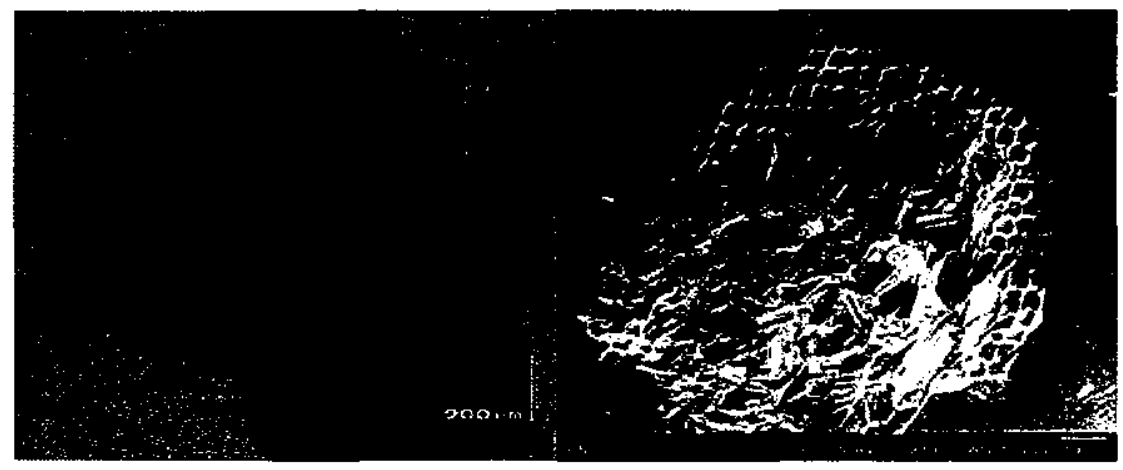

Figure 4.3: $532 \mathrm{~nm}$ ns pulse laser ablated polycrystalline diamond under optical microscope (left) and Scanning Electron Microscope (SEM) (right). (These pictures are taken by J. Smedley and T. Rao)

Figure 4.4 shows the images of polycrystalline diamond after ablation with $266 \mathrm{~nm} \sim 10 \mathrm{~ns}$ pulse duration laser. Both images show clearer raster scan pattern as well as some dark areas, and the surface damage caused by $266 \mathrm{~nm}$ laser is limited to small craters scattered on the ablated area. 


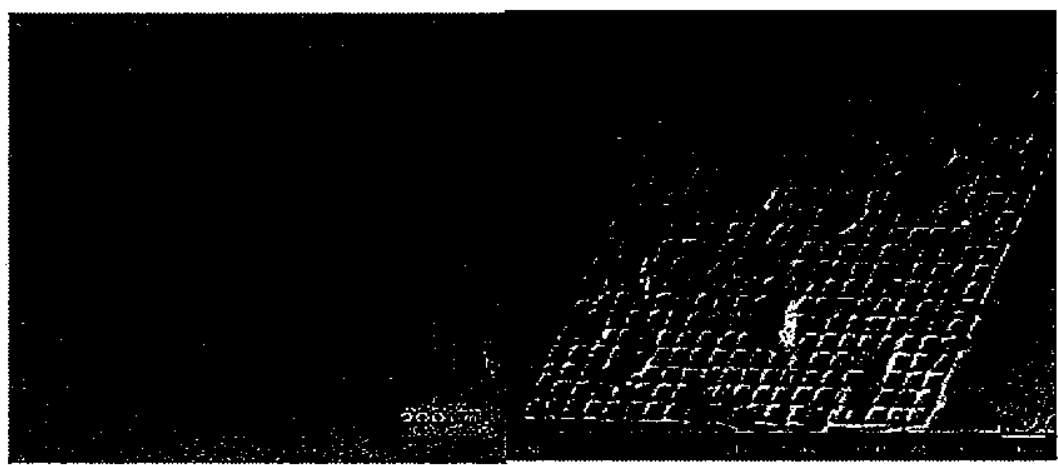

Figure 4.4: 266nm ns pulse laser ablated polycrystalline diamond under optical

microscope (left) and Scanning Electron Microscope (SEM) (right). (These pictures are taken by J. Smedley and T. Rao)
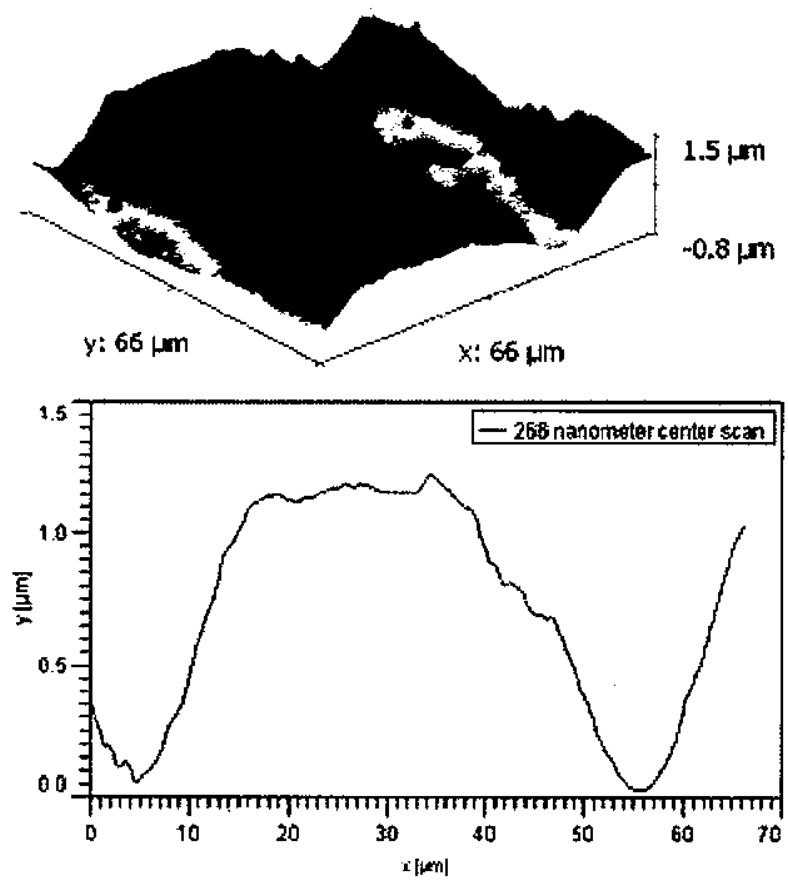

Figure 4.5: AFM image of polycrystalline diamond $266 \mathrm{~nm}$ laser ablated area center. 


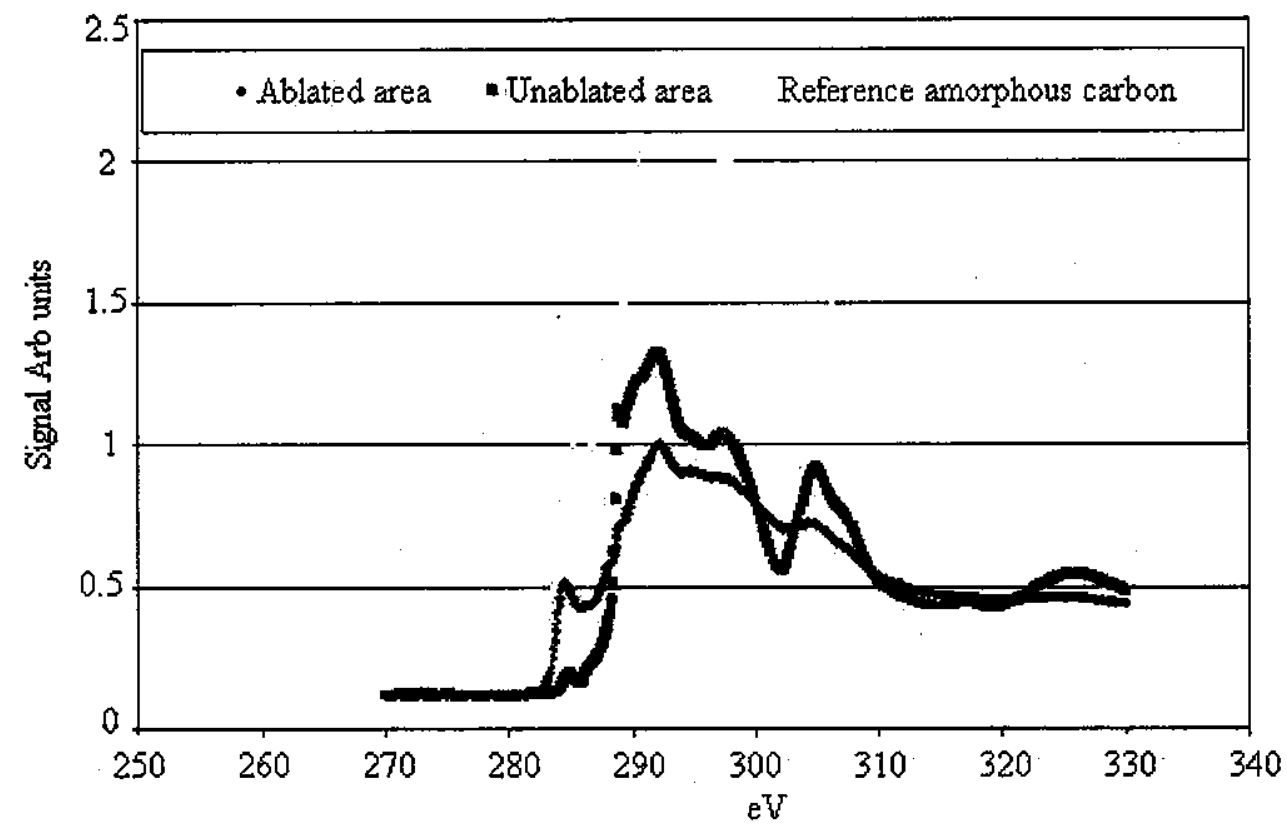

Figure 4.6: NEXAFS trace of unablated and ablated regions of diamond sample and reference trace for amorphous carbon. (These curves are obtained by J. Smedley)

AFM image shows the ablated area has about $2 \mu \mathrm{m}$ roughness. The clear trace of the raster scanning is unacceptable for thinning the diamond amplifier, where the roughness should be controlled to under $1 \mu \mathrm{m}$.

NEXAFS from the unablated and ablated sections of the diamond are shown in Figure 4.6. NEXAFS is a highly sensitive surface technique that can clearly delineate surface impurity from the bulk impurity and can identify different forms of carbon unequivocally. A reference curve for the amorphous carbon is also shown in the same plot. In comparison to the unablated region, the ablation process has increased the relative strength of the peak at $285 \mathrm{eV}$ while reducing the strength of the peaks at 
$289 \mathrm{eV}$ and beyond $292 \mathrm{eV}$. The strong similarity between the reference plot and the NEXAFS signal from ablated section of the dia mond imply that the darkened region is predominantly amorphous carbon (AC). The AC adheres on the surface of the diamond, and can be easily removed chemically or in an ozone environment.

To form a smooth surface after the ablation, the raster scan size is decreased to $5 \mu \mathrm{m}$ to overlap the adjacent ablation traces. The laser is changed to $213 \mathrm{~nm}$, and the pulse duration is decreased to $\sim 30 \mathrm{ps}$ to deposit a comparable energy to the diamond sample as ablations above. $266 \mathrm{~nm}$ photon is corresponding to $4.7 \mathrm{eV}$ energy, which is below the band gap of the diamond where $213 \mathrm{~nm}$ photon is corresponding to $5.8 \mathrm{eV}$ energy, which is just above the band gap. Thus $213 \mathrm{~nm}$ laser is opaque to diamond.

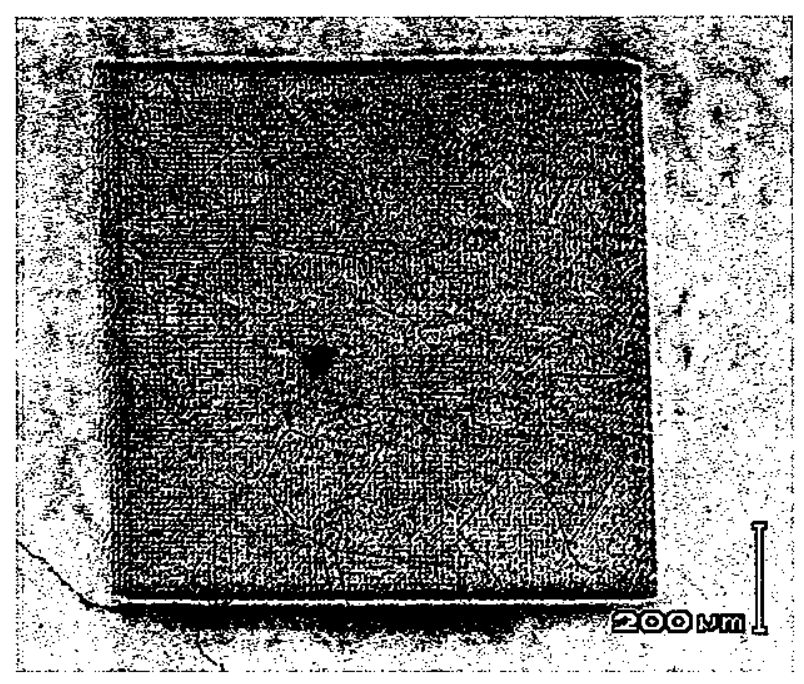

Figure 4.7:213nm ns pulse laser ablated polycrystalline diamond under optical microscope. (This picture is taken by J. Smedley and T. Rao) 

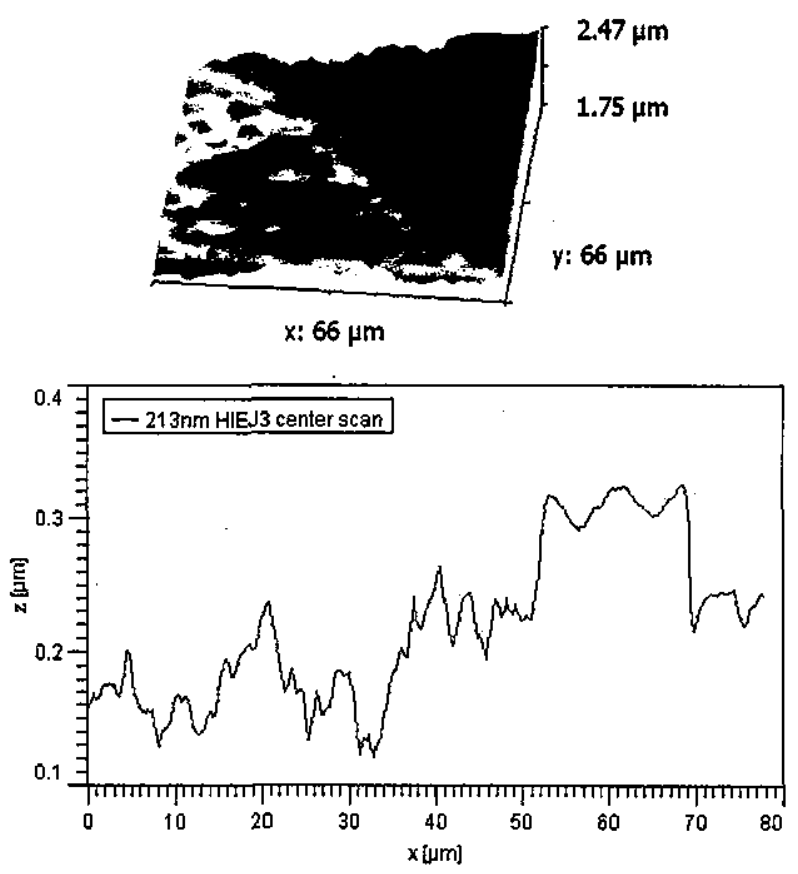

Figure 4. 8: AFM image of polycrystalline diamond $213 \mathrm{~nm}$ laser ablated area center.

The fine lines shown in Figure 4.7 within the ablated area are grain boundaries of the polycrystalline diamond, which is same as the well defined sharp-angled pattern shown in Figure 4.8. The area within the sharp angle has more material left after ablation than the rest of the area, which is differently oriented. This indicates the energy absorbed by one orientation is different from another orientation. So to obtain a smooth surface, single crystal diamond must be applied.

\subsubsection{Ablation in rich $\mathrm{O}_{2}$ environment}

The vacuum chamber was backfilled with oxygen at base pressures of $14.2 \mathrm{Torr}$, 
0.9 Torr, 0.1 Torr and $<0.01$ Torr using a leak valve and diamond was ablated at each of these pressures. Optical transmission measurements using 623.8nm HeNe laser and optical constants of amorphous carbon indicate that the thickness of the non-diamond carbon layer is $\sim 130 \mathrm{~nm}$ and does not depend strongly on the oxygen pressure. The Raman spectra for the ablated surfaces are shown in Figure 4.9. As can be inferred from the data, the presence of oxygen did not make a difference to the formation of the non-diamond carbon on the ablated surface.

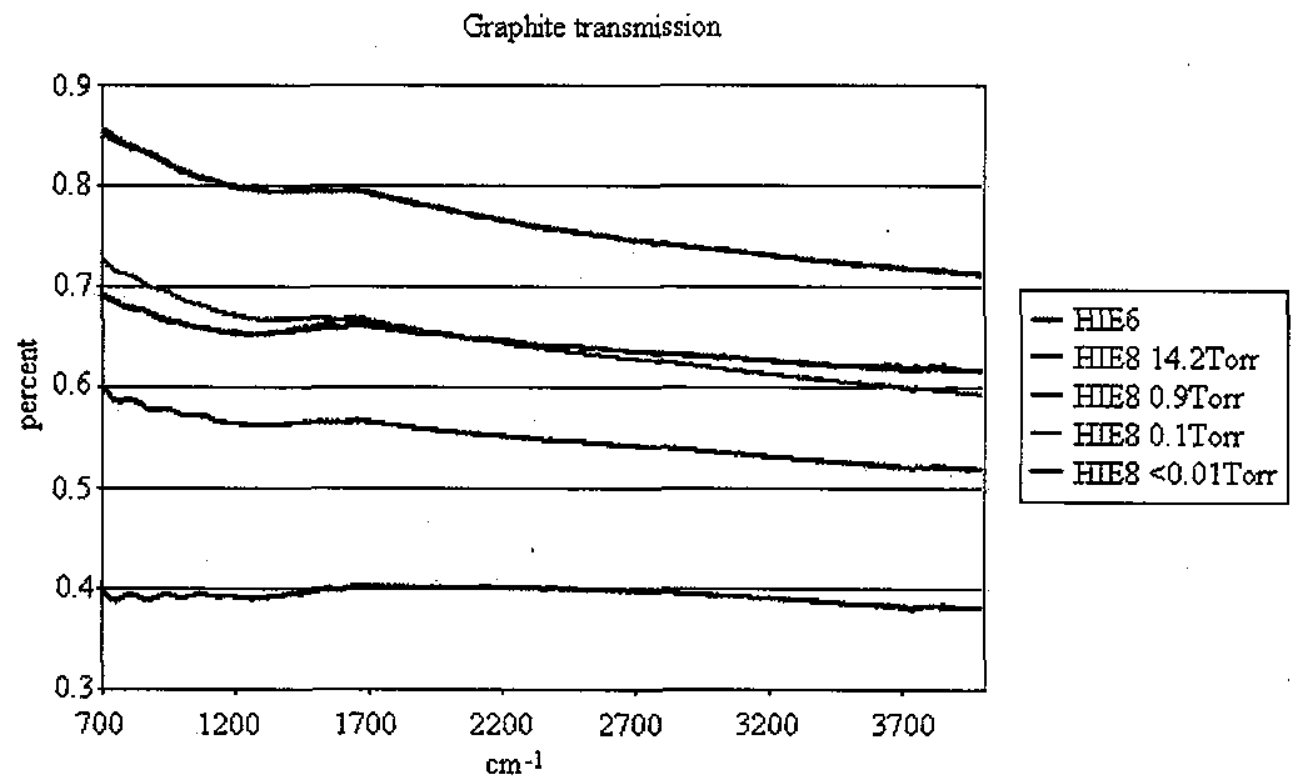

Figure 4.9: Raman spectra of samples following ablation with different $\mathrm{O} 2$ pressures.

(These curves are obtained by J. Smedley)

\subsubsection{UV exposure}

The ablated surface of HIE8 was exposed to the radiation from a mercury arc 
lamp (Jelight, GLF-12-SRC) for 6 hours at a distance of $5 \mathrm{~cm}$ from the lamp.

FTIR Transmission measurements and theoretical fit for graphite and Amorphous Carbon ( $\mathrm{AC}$ )

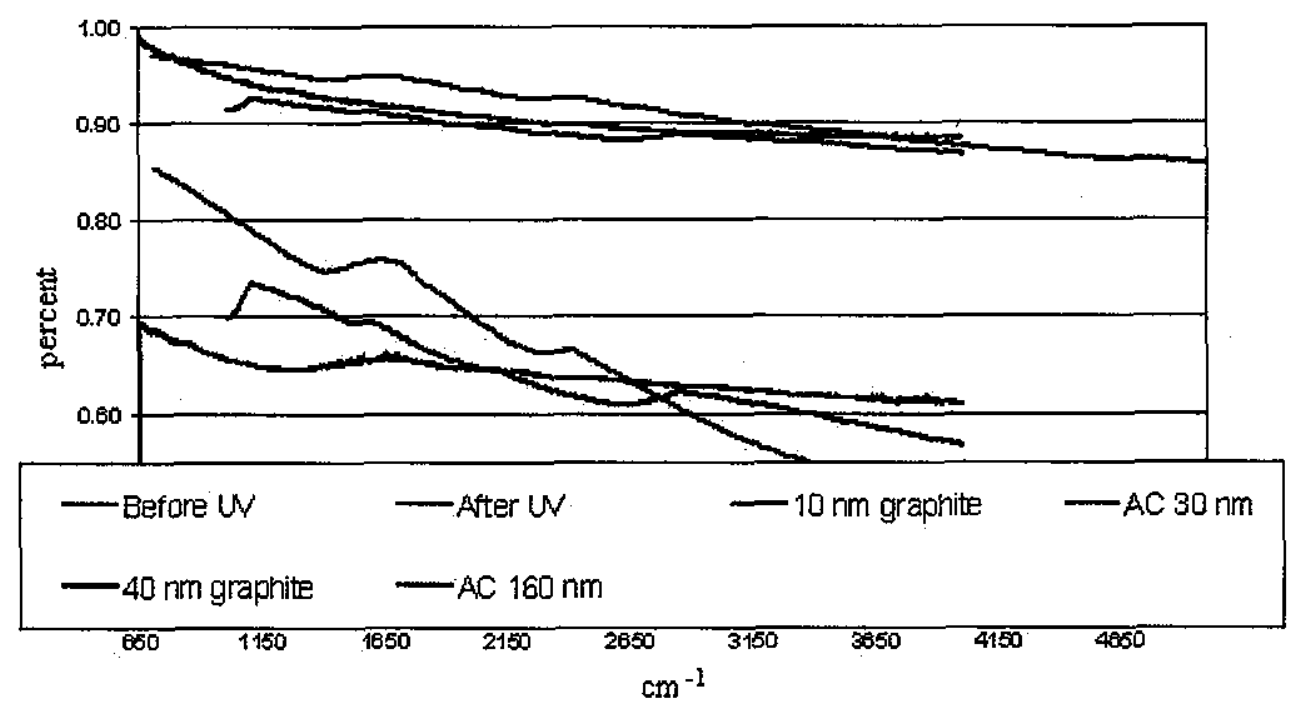

Figure 4.10: FTIR spectra of before and after UV exposure of ablated area. Different thickness of graphite and $\mathrm{AC}$ theoretical absorption curves are also shown as reference. (These curves are obtained by $J$. Smedley)

In the region of wave numbers from $800 \mathrm{~cm}^{-1}$ to $4000 \mathrm{~cm}^{-1}$, the IR transmission has changed from $65 \%$ before the UV radiation to $94 \%$ after the radiation. The UV radiation in air would create a reactive ozone environment. Ozone would then form $\mathrm{CO}_{2}$ at the presence of non-diamond carbon and left the surface cleaned. 


\subsection{Laser ablation of single crystalline diamond}

The dark areas and uneven surface on ablated areas of polycrystalline diamond could have been due to the grain boundaries. A single crystal was ablated with $213 \mathrm{~nm}$ ps laser. This energy is above the band gap of the diamond, so the diamond is opaque to the wavelength and all the energy will be deposited at the surface.

A single crystal diamond sample (HID13) was ablated using a laser pulse of $100 \mu \mathrm{J}$ energy at a wavelength of $213 \mathrm{~nm}$ and pulse duration of $30 \mathrm{ps}$ at a repetition rate of $10 \mathrm{~Hz}$. The sample was raster scanned with $5 \mu \mathrm{m}$ step size. The optical microscope pictures of the ablated and unablated regions are shown in Figure 4.11. 


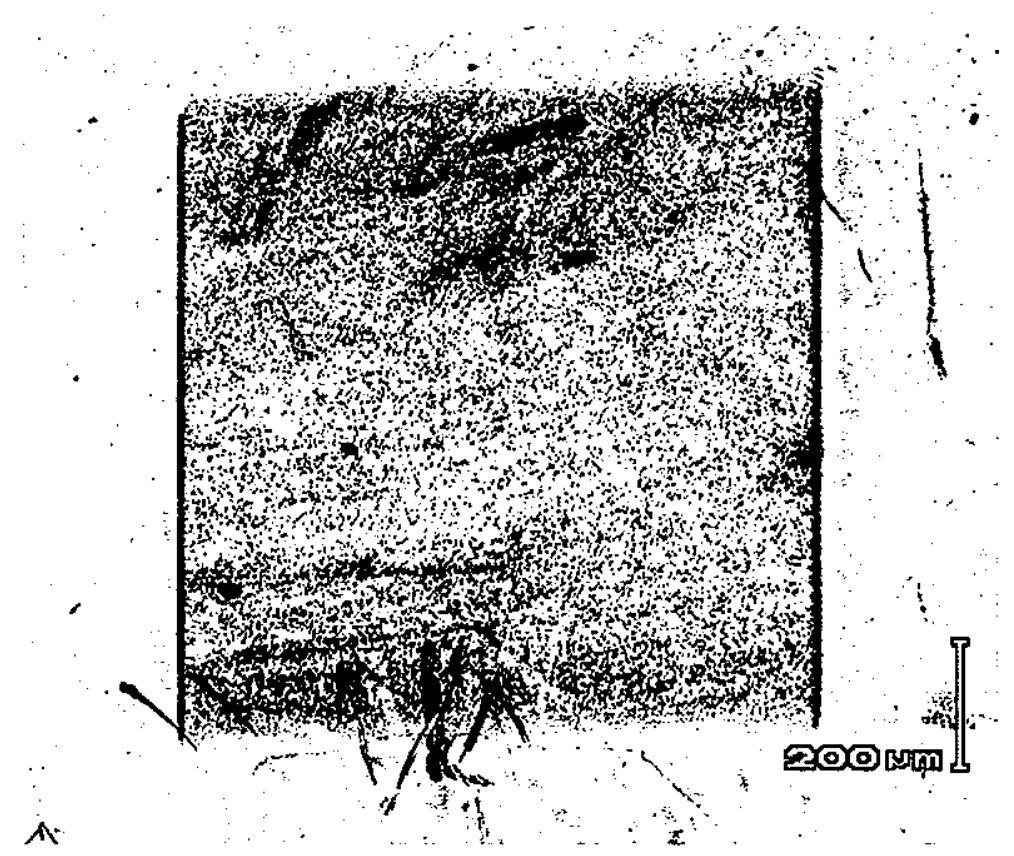

Figure 4.11: Optical microscope picture of $213 \mathrm{~nm}$ laser ablated single crystal diamond. (This picture is taken by J. Smedley and T. Rao) 

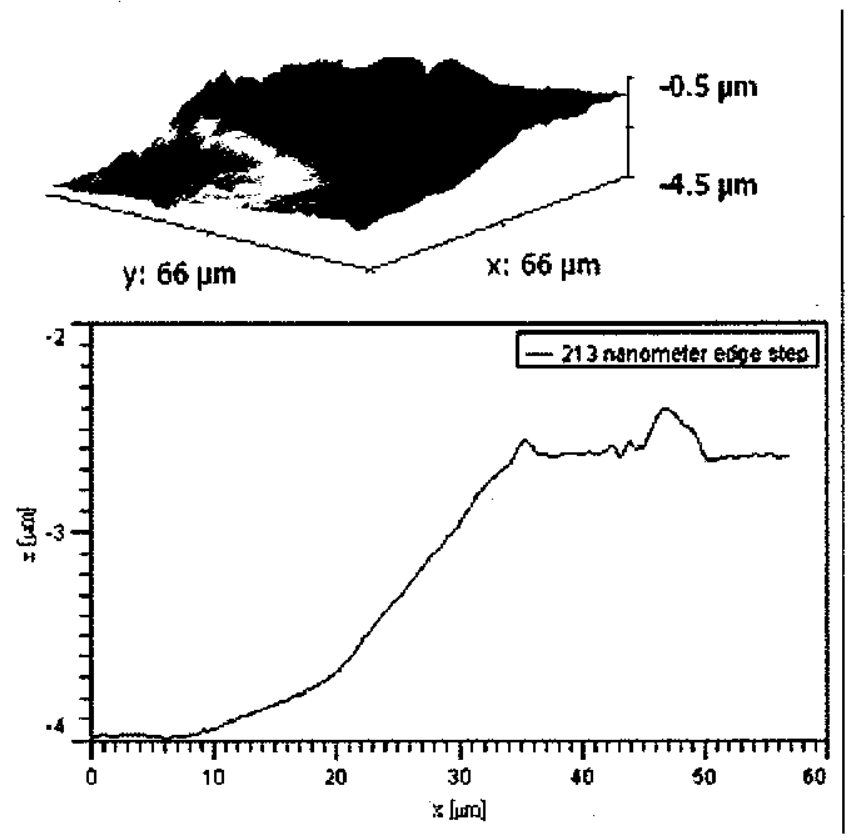

Figure 4.12:AFM image of single crystal diamond 213nm laser ablated area edge.
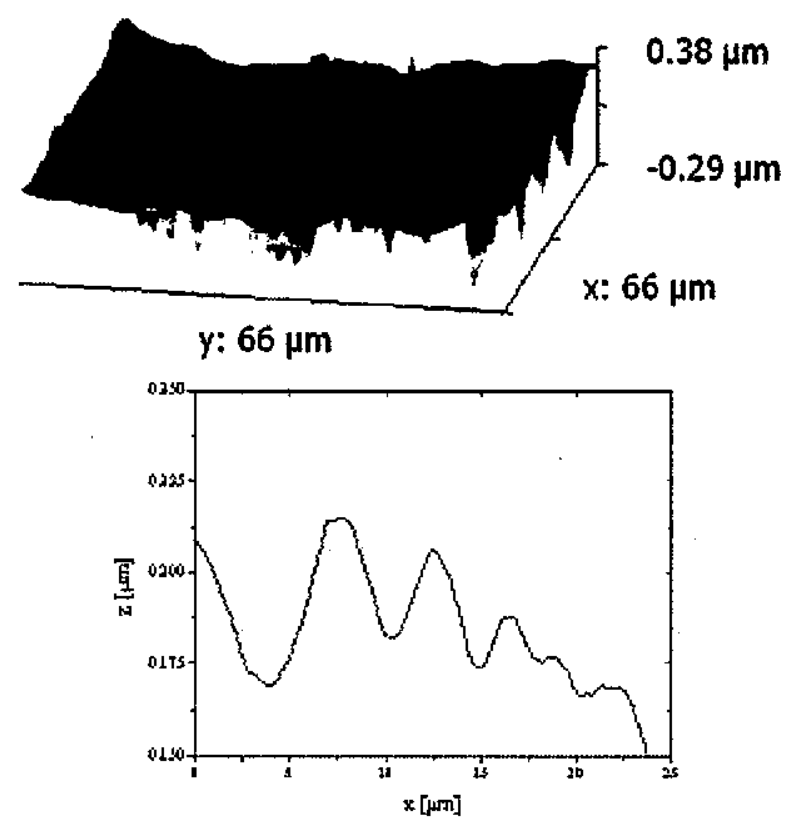

Figure 4.13: AFM image of single crystal diamond 213nm laser ablated area center. 
The roughness in Figure 4.13 is about $100 \mathrm{~nm}$, which is tolerable for the amplifier application. Much less dark areas are created in this ablation process, and the raster scanning pattern is hard to distinguish from the AFM images. From Figure 4.11, the optical microscope image shows that there is much less surface non-diamond carbon coverage than Figure 4.3 and Figure 4.4, and it does not show the grain boundaries as Figure 4.7.

Laser ablation with energy above the diamond band gap is proved to be a feasible method of thinning the diamond with submicron roughness with only a very small amount of non-diamond carbon left on the surface. A RIE process can be added to refine the surface of the laser ablated surface. The combination of both processes is ideal for thinning the diamond sample to a few tens of microns with surface roughness in nanometer scale. 


\section{TRANSMISSION MODE MEASUREMENT OF DIAMOND SECONDARY ELECTRONY IELD}

Transmission mode measurements are designed to study the secondary electron generation and transport in the diamond. The emission into the vacuum uses a different experimental setup and will be considered in Chapter 6 .

\subsection{Measurement setup}

\subsubsection{Primary electron source}

Primary electrons are generated from a thermionic electron gun source Model EGG-3101 commercially purchased from Kimball Physics. The cathode material is high-brightness single-crystal lanthanum hexaboride $\left(\mathrm{LaB}_{6}\right)$ with small spot size option, which can provide $10 \mu \mathrm{A}$ peak current maximum and $100 \mu \mathrm{m}$ diameter spot size minimum with optimum working distance. The gun has up to $10 \mathrm{keV}$ output electron energy. The electron energies selected for experiments are limited to $8 \mathrm{keV}$ to allow a safety margin. 


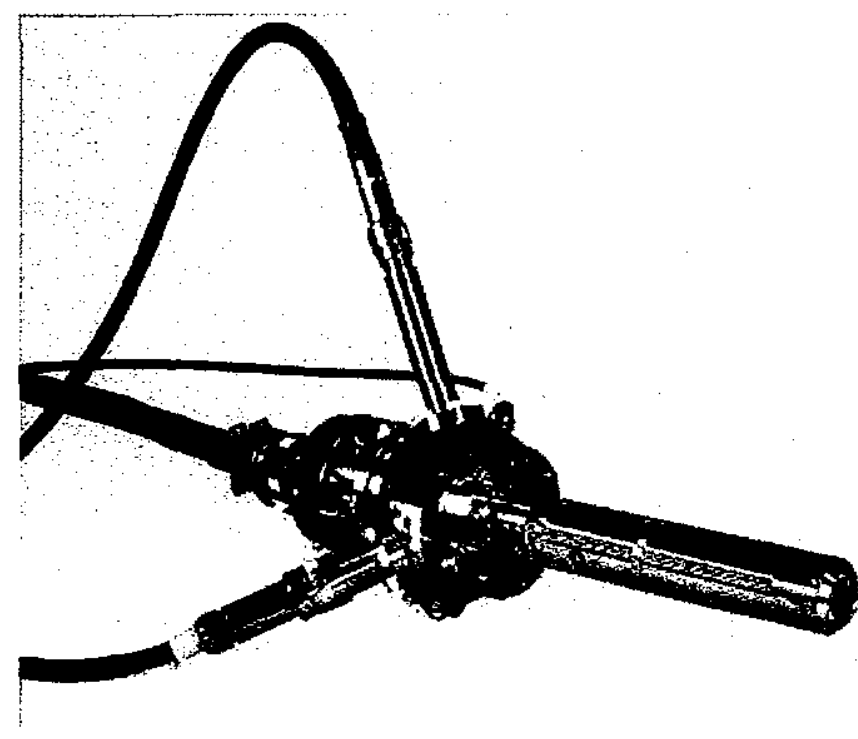

Figure 5.1:EGG-3101 thermionic electron gun from Kimball Physics.

Primary electrons are focused by an Einzel lens structure panels inside the gun.

With the available electron blanking option, the primary beam from the gun can be pulsed. For short pulse output, an electric circuit is designed to trigger the blanker inside the gun. Using the delay function of each output of the Stanford DG535 Digital Delay and Pulse Generator shown in Figure 5.2, two successive pulses can be sent out with programmed timing. These two successive pulses can be delivered when needed to study the electron or hole trapping in diamond. 


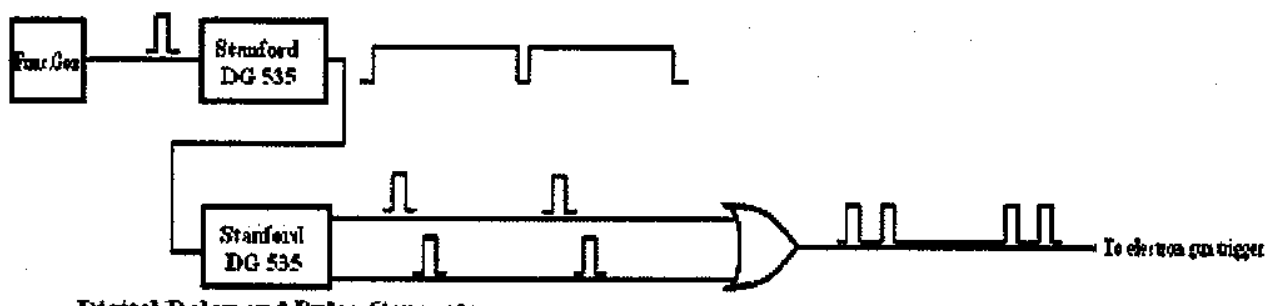

Digtal Delay and Pulse Gemetanor

Figure 5.2: Sketch of electron gun pulsing trigger.

\subsubsection{Diamond holder and system setup}

During the measurement, a sample holder is used for three purposes: Supporting the diamond surface perpendicular to the primary beam; insuring electrical contact to the primary electron impact surface of diamond in order to provide a replenishment current; and providing an electric field to accelerate the secondaryelectrons and allow measurement of the secondary current.

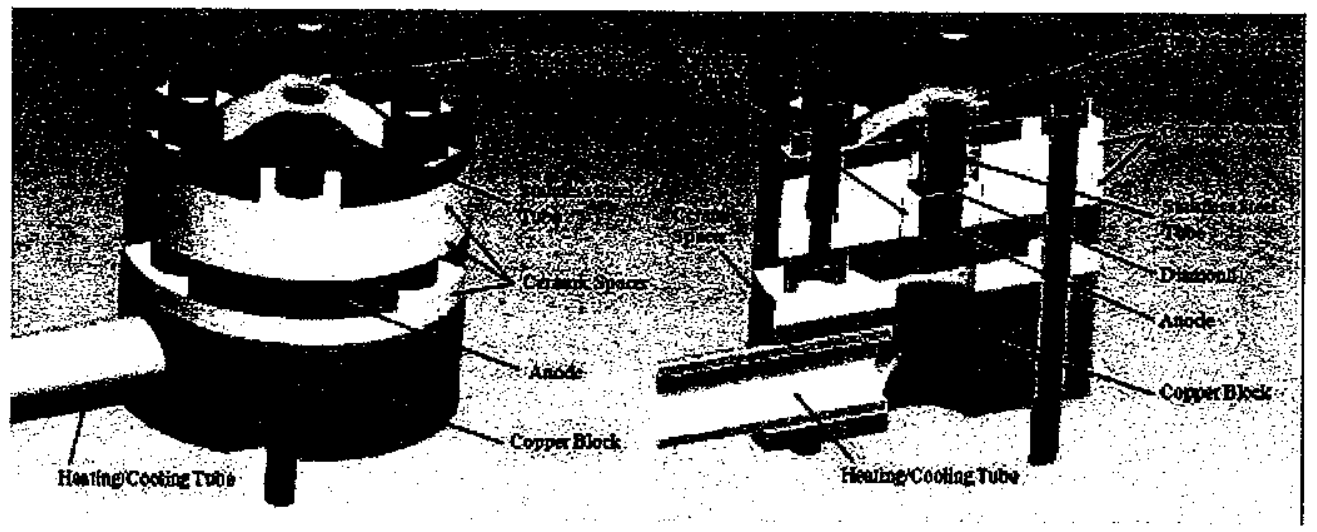

Figure 5.3: Diamond holder structure. (Created by Chong-Jer Liaw) 
In transmission mode measurements, the diamond is coated with selected thin metal films, identically on both sides. A stainless steel tube is pressed onto the primary electron impacting surface (primary surface), and the inner diameter of the tube is slightly less than the metal coating outer rim. . The tube is then connected to electrical ground through a current measurement resistor. The voltage on the resistor is typically $10 \mathrm{~s}$ of millivolts and therefore the primary surface can be considered effectively grounded. Good electrical contact is ensured between the metal coa ting and the tube to provide replenishment currents. The primary side of the diamond is grounded through the oscilloscope during all studies and the signal is measured on this low voltage level.

On the other side of the diamond, a copper anode is in good electrical contact with the metal coating. The anode is connected to a high voltage power supply, and it can be set to any voltage between $0 \mathrm{~V}$ to $5000 \mathrm{~V}$. The limit of $5000 \mathrm{~V}$ is governed by the potential breakdown, affecting the vacuum level of the system and for safety considerations. Electrical break down through the vacuum might happen if the voltage is exceeds $5000 \mathrm{~V}$, which corresponds to more than $15 \mathrm{MV} / \mathrm{m}$.

Ceramic spacers and washers are used as insulators given their good performance in ultra-high vacuum. The use of the ceramic spacers also makes the insulation path along the insulator's surface very long and the insulator's surface is not directly exposed to the electron beams. These are very important considerations to improve the insulation ability. 
A copper block of relatively large mass with a stainless steel tube connected is at the very back of the holder. The entire stack of components is screwed down onto this block. The long tube which extending out of the system can provide heating and cooling for different experimental conditions. Liquid nitrogen can be fed through the tube and bring the system down to less than $-140^{\circ} \mathrm{C}$. A cartridge heater is also available to slide down the tube to bring the system up to greater than $200^{\circ} \mathrm{C}$.

A leak checking is done on the whole assembly, and the vacuum can reach 2E-9Torr after baking.

The principle of the transmission mode experiment is shown in Figure 5.4.

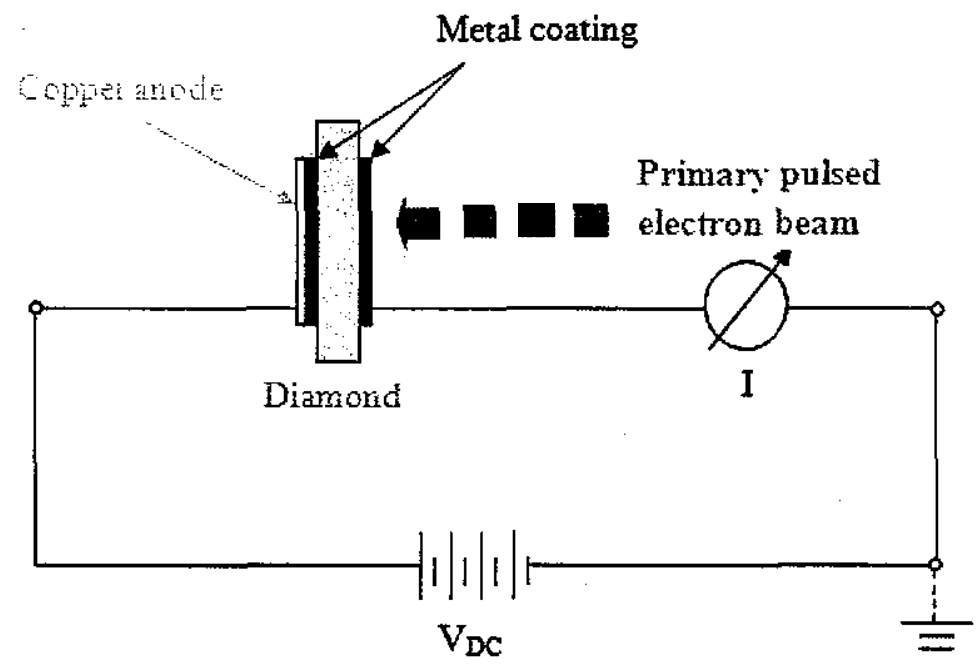

Figure 5.4: Simple sketch of the transmission mode measurement principle.

Either side of the high-voltage supply can be grounded.

$V_{D C}$ is the applied high voltage on the anode. Current $I$ measures the 
replenishment current as the secondary electrons transits through the dia mond. The SEY of the amplifier is calculated by comparing the ratio between the replenishment current and the primary current.

\subsubsection{Signal collection circuit}

According to the sample holder design for transmission mode measurement, high voltage is applied to the copper anode for secondary electron acceleration and collection. As the secondary electrons move away from the injection surface to the opposite electrode and leave the diamond, replenishment current enters the diamond from the ground electrode and can be detected by the oscilloscope connected to the holder. The signal collection circuit needs to have a fast response and good sensitivity to small signals. The signal cable is kept short to reduce the distributed capacitance.

\subsection{Experimental results}

The electrode of the primary electron side of the holder is designed with a tubular shape (similar to a Faraday Cup) to collect most of the secondary electrons that are generated by the primary electrons on the metal coating surface and escape to vacuum. It is found that more than $90 \%$ of those electrons are captured by the electrode when the primary beam is focused in that hole. The primary electron current is measured by focusing the beam inside the hole and applying no field on the 
diamond.

With the experiment setup discussed in Section 5.1.2, pulsed secondary electron signals are measured by oscilloscope. The signal is then compared with the primary electron signal. The ratio between the secondary current and primary current will give the SEY under the given applied electrical field in the diamond.

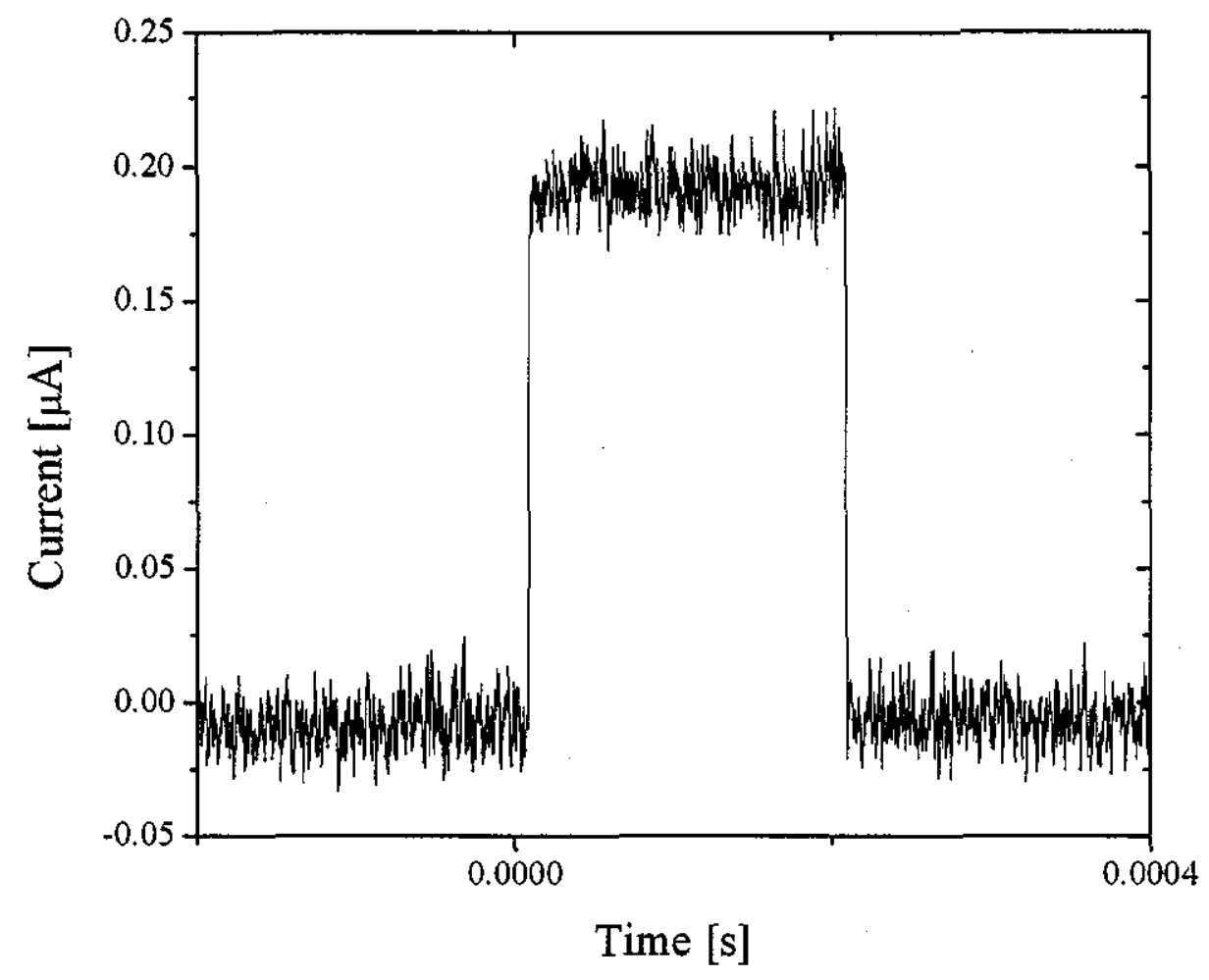

Figure 5.5: Primary electron signal with pulse width of 20qus.

Under a low applied field, the secondary electron drift velocity is not saturated. The electron thermal energy at equilibrium is also smaller than that at higher applied field and therefore under a low applied field these electrons have a larger probability 
of being trapped. The trapped electrons will form an electric field, opposing the applied field. The opposing field causes the succeeding electrons to experience a weaker accelerating field. Therefore, fewer electrons will be able to leave the plasma region and reach the anode, i.e. we observe a decrease of the signal or SEY. If the pulse is adequately long, the effective field will decrease continuously in amplitude as more and more electrons are trapped. Eventually, the equilibrium state would be reached when the trapped electron field fully cancels the applied field, and no more secondary electrons will be able to leave the plasma range. Efforts to reduce trapping will be further discussed in Section 5.3.

By using high-purity single crystal diamonds, the trapping centers in the diamond bulk are limited. At a high applied field situation, the field decrease due to the trapping is small compared to the applied field. The decrease of amplitude in each pulse will be negligible. 

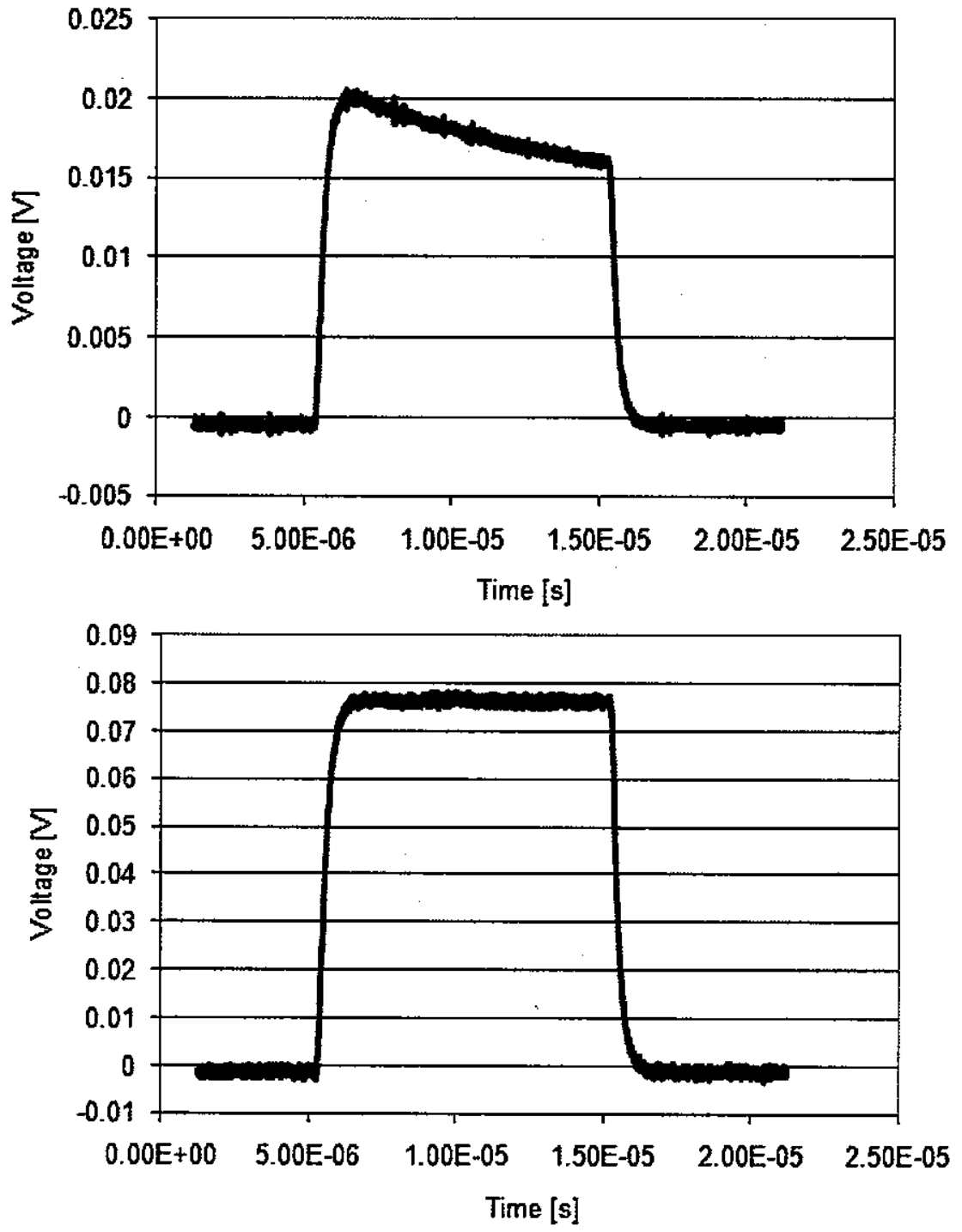

Figure 5.6: Comparison of low field (top: $0.3 \mathrm{MV} / \mathrm{m}$ ) and high field (bottom: $3 \mathrm{MV} / \mathrm{m}$ ) secondary electron signal of single crystal diamond with $0.3 \mathrm{~mm}$ thickness in transmission mode.

The SEY or gain can be calculated by: 
gain=secondary current signal amplitude / |primary current signal amplitude $\mid+1$

The sign of the additional term equal to 1 added at the end of Equation (5.1) is to reflect the negative polarity of the primary current signal.

Gain vs. field inside the diamond sample can be plotted as shown in Figure 5.7, and this plot is a good tool for explaining the performance of the diamond amplifier [23]. 


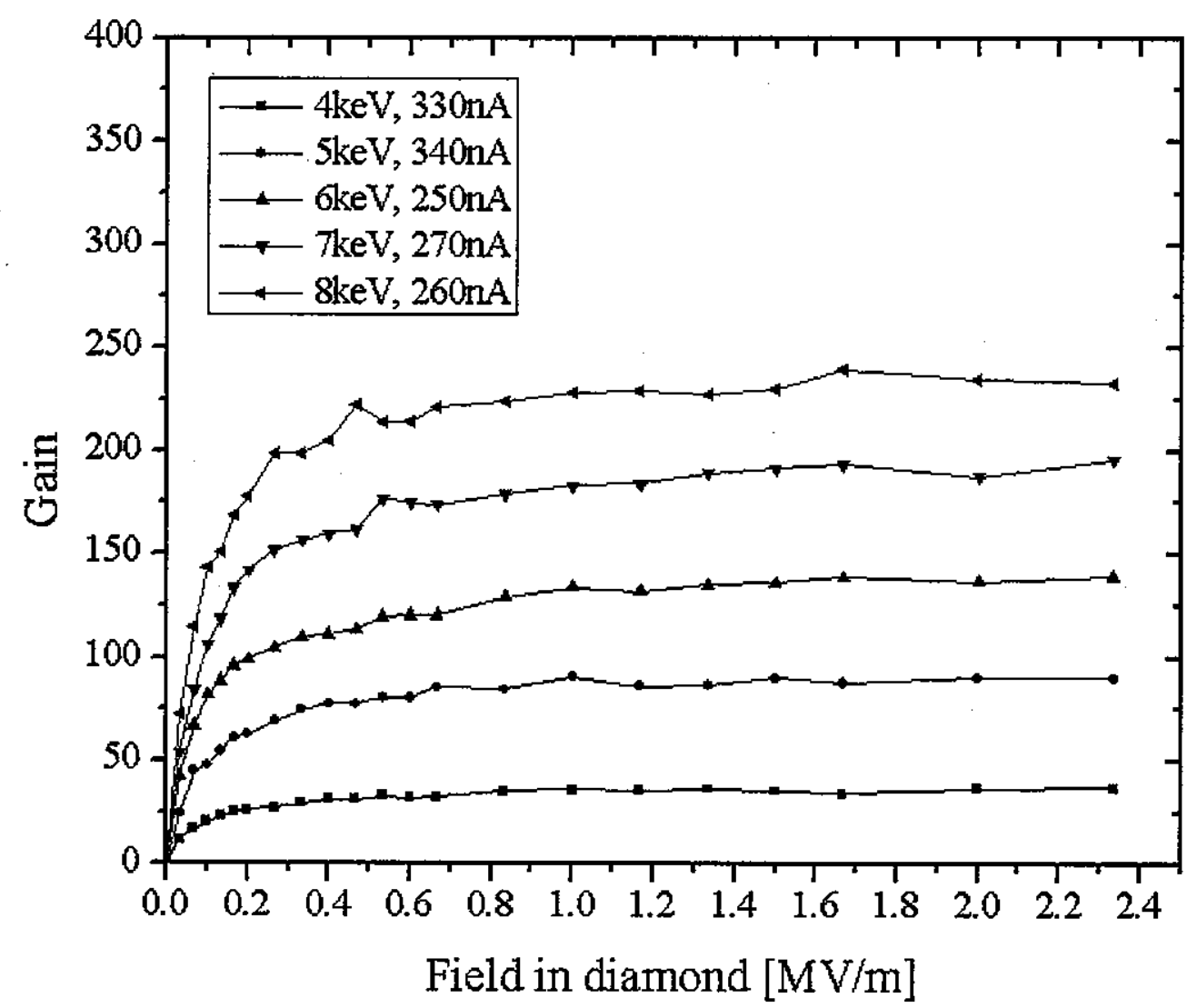

Figure 5.7: Single crystal diamond window gain with primary electron energy ranges from $4 \mathrm{keV}$ to $8 \mathrm{keV}$. Primary currents are shown in the legend.

The plasma formed as each primary electron penetrates through the diamond comprises electron-hole pairs. These will be separated under the applied field. With no applied field the electrons and holes will all recombine with three-body scattering or diffuse into the metal coating, leading to no gain. The electrons that make it through the plasma region without recombination (or trapping) and arrive at the opposite surface will contribute to the gain. According to each curve in Figure 5.7, the 
gain started with a small value under low electric field in diamond, which shows that the recombination is significant. With the increase of the applied field, more electrons can move away and the time needed for total separation decreases, thus the gain increases. As the applied field increases, the charge carriers reach the saturation drift velocity and the gain saturates. The gain under saturation only depends upon the primary electron energy. Detailed explanation of recombination will be discussed in Section 5.5.

With $8 \mathrm{keV}$ primary electron energy, the saturated gain is nearly 250 . This indicates that the diamond amplifier has the capability of increase the input current by two orders of magnitude. The minimum applied field needed for reaching saturated gain is less than $0.5 \mathrm{MV} / \mathrm{m}$, which corresponding to $150 \mathrm{~V}$ on the anode in our setup.

The signal in a pulse dropping continuously for low field is due to trapping of the electrons, and this can be avoided by detrapping methods that will be discussed in the next Section. With a relatively fast-response circuit, the value of the peak in the beginning of the signal corresponds to the number of electrons that are pulled out of the plasma. Thus the value of the signal used for calculating the gain is taken at the peak of the pulse in soon after its onset.

\subsection{Laser Detrapping}

Due to the trapping mechanism diamond can easily have excessive electrons held 
within potential wells. These electrons will create electric field with opposite polarity to the applied field. The trapping centers can be impurities, grain boundaries, distortion of the crystal lattice, etc., and can be decreased by using high purity single crystal diamond samples.

However, during sample preparation process, the diamond is subjected to high temperature, surface etching and metallization. These aggressive procedures may introduce stress or impurities into the sample, and introduce trapping centers.

The probability of electrons being trapped by the trapping centers is constant, given the large number of potential trapping centers. Thus, as current flows into the diamond, the number of trapped electrons will increase with time. Figure 5.6 shows the decrease of gain within the first pulse. Under a continuous pulse mode with high repetition rate, the succeeding pulses will all be affected by the electrons trapped during the preceding pulses. The electrons also have the possibility of escape from the trapping centers due to applied electric field and thermal activity. So the escape probability increases with increasing anode voltage and the time with current off between pulses. If the applied field is high enough and/or time between pulses is long enough to make the number of electrons released from the trapping centers comparable with the number of electrons that are being trapped in one pulse, the signal will reach equilibrium. The equilibrium state is also dependent of the current density of the primary beam. Higher current density will result in more electrons trapped in the diamond. 


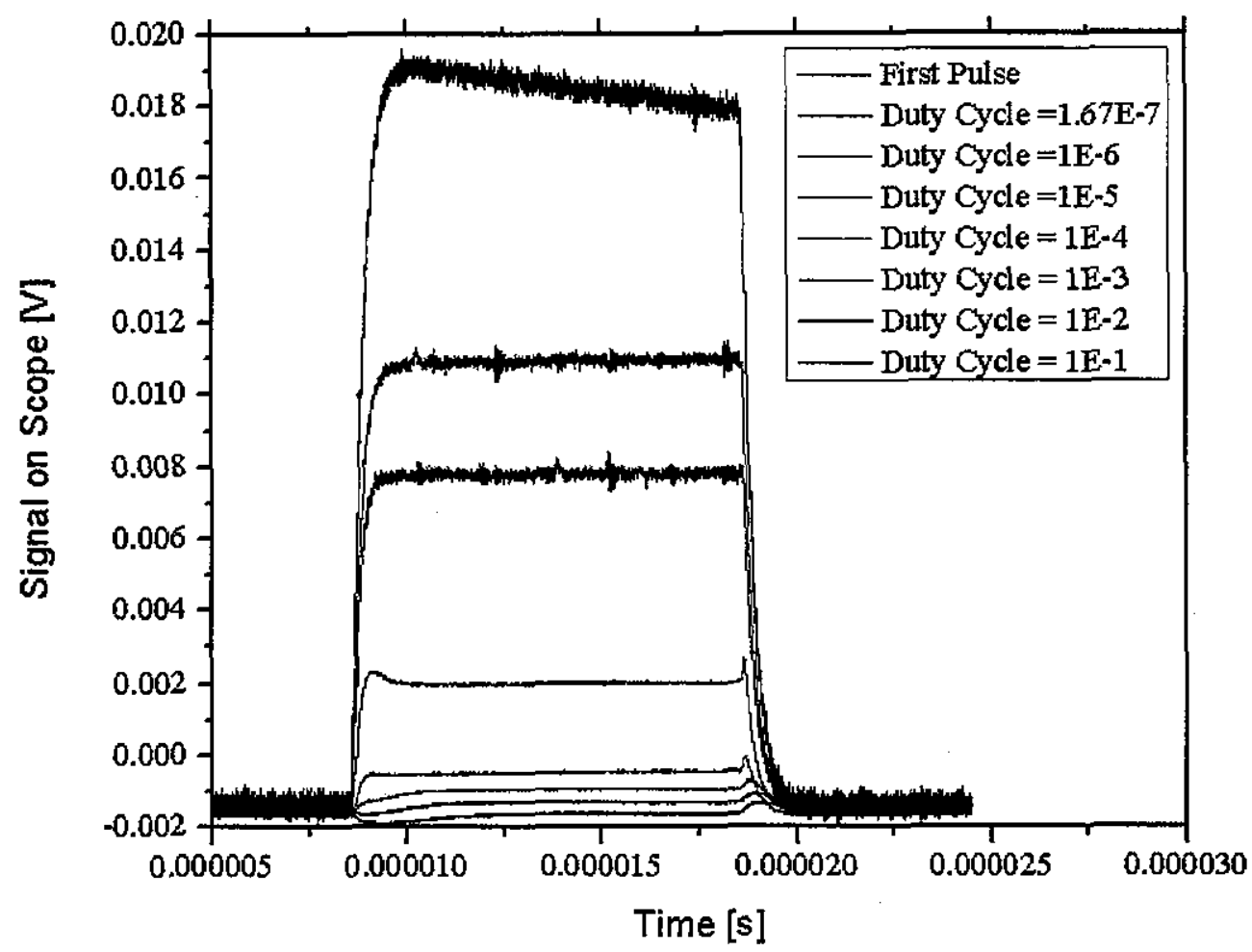

Figure 5.8: Secondary electron signal of 10us pulse width with different duty cycles.

No laser beam applied. Primary electron peak current density is $0.03 \mathrm{~A} / \mathrm{m}^{2}$ and the field applied in the diamond is $0.033 \mathrm{MV} / \mathrm{m}$.

Detrapping of the electrons in a transmission mode is made possible by applying a laser beam to the system during the experiment. Photons will excite the trapped electrons. If the applied field is set to zero, these exited electrons will move under the field of the trapped electrons. Eventually all the trapped electrons would be de-trapped.

Most of the trapping centers in our diamond samples are rather shallow. Photons 
of $532 \mathrm{~nm}$ (green) wavelength, which is $2.34 \mathrm{eV}$ in energy, are applied to observe a significant change in gain under relatively low field. A laser pointer with $<5 \mathrm{~mW}$ power is used to provide the detrapping light, and a slot was opened on the holder along the path of the light to allow the photons to reach and interact with the diamond.

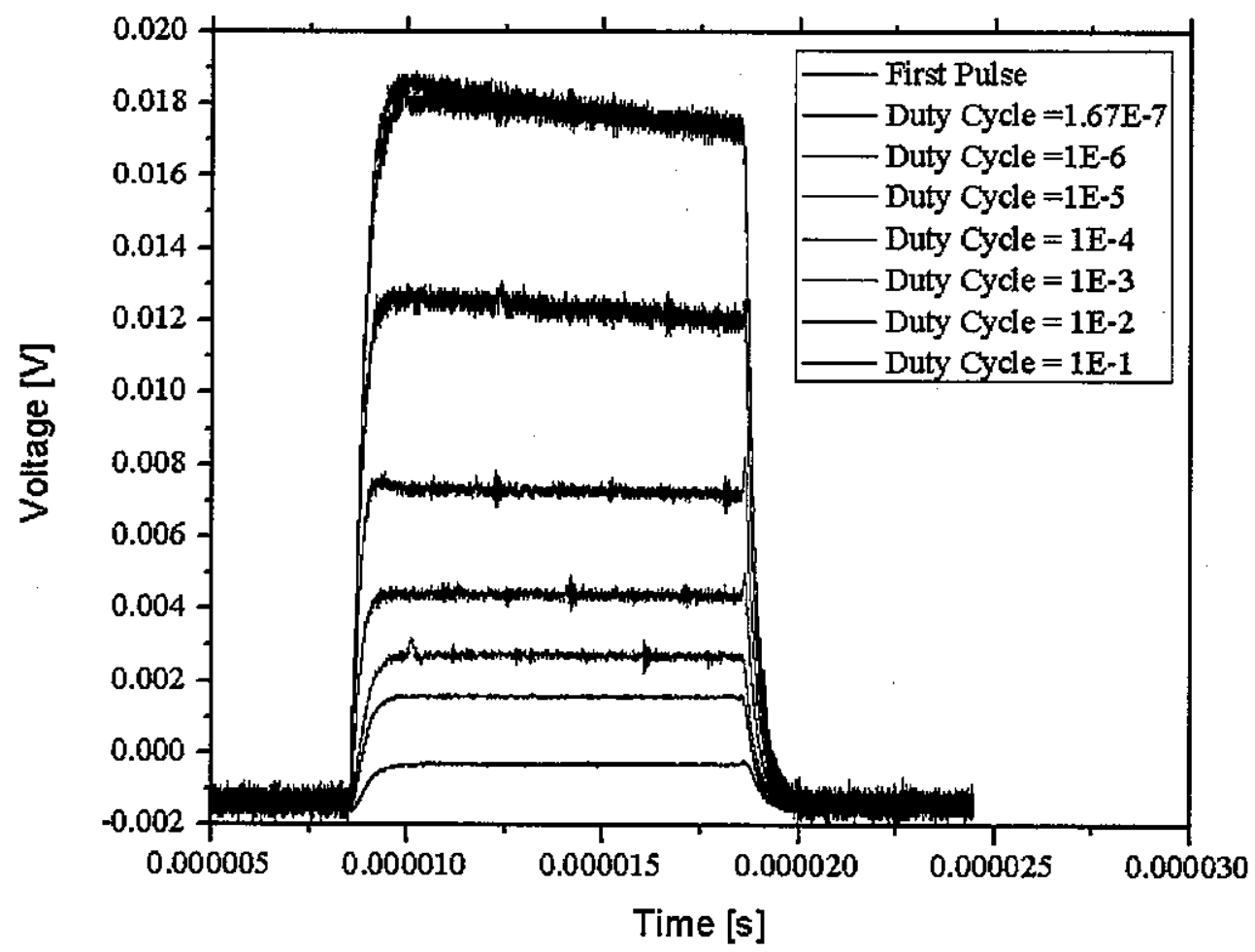

Figure 5.9: Secondary electron signal of 10us pulse width with different duty cycles.

Laser beam applied $(532 \mathrm{~nm},<5 \mathrm{~mW})$. Primary electron peak current density is $0.03 \mathrm{~A} / \mathrm{m}^{2}$ and the field applied in the diamond is $0.033 \mathrm{MV} / \mathrm{m}$.

By comparing the corresponding curves in Figure 5.8 and Figure 5.9, it is obvious that laser beam played an important role in increasing the signal by releasing 
the electrons from the trapping centers. Both figures show that the smaller the duty cycle, i.e. more time between pulses, the larger the signal. The following sections will discuss laser detrapping under different conditions as well as field dependence.

\subsubsection{Current Density}

The total number of trapped electrons is determined by the product of probability of trapping and the charge density within the trapping region in the diamond. Without laser detrapping, for higher primary current density, more electrons will be trapped. The signal observed after equilibrium is much lower than the first pulse unless the applied field is extremely high. The time required for the system to reach equilibrium varies with current density, but is within the range of 1 minute under all of our experimental conditions.

The primary beam energy and spot size is fixed using the control system of the gun. The peak of the first pulse is recorded as the total number of electrons created, which should be the maximum gain under such applied field. The beam is pulsed at $10 \mathrm{~Hz}$ repetition rate and duty cycle of $0.01 \%$.

The applied field is increased from $0 \mathrm{MV} / \mathrm{m}$ to above $1.8 \mathrm{MV} / \mathrm{m}$, and signals under the equilibrium state are recorded for gain calculation both with and without the laser beam. 

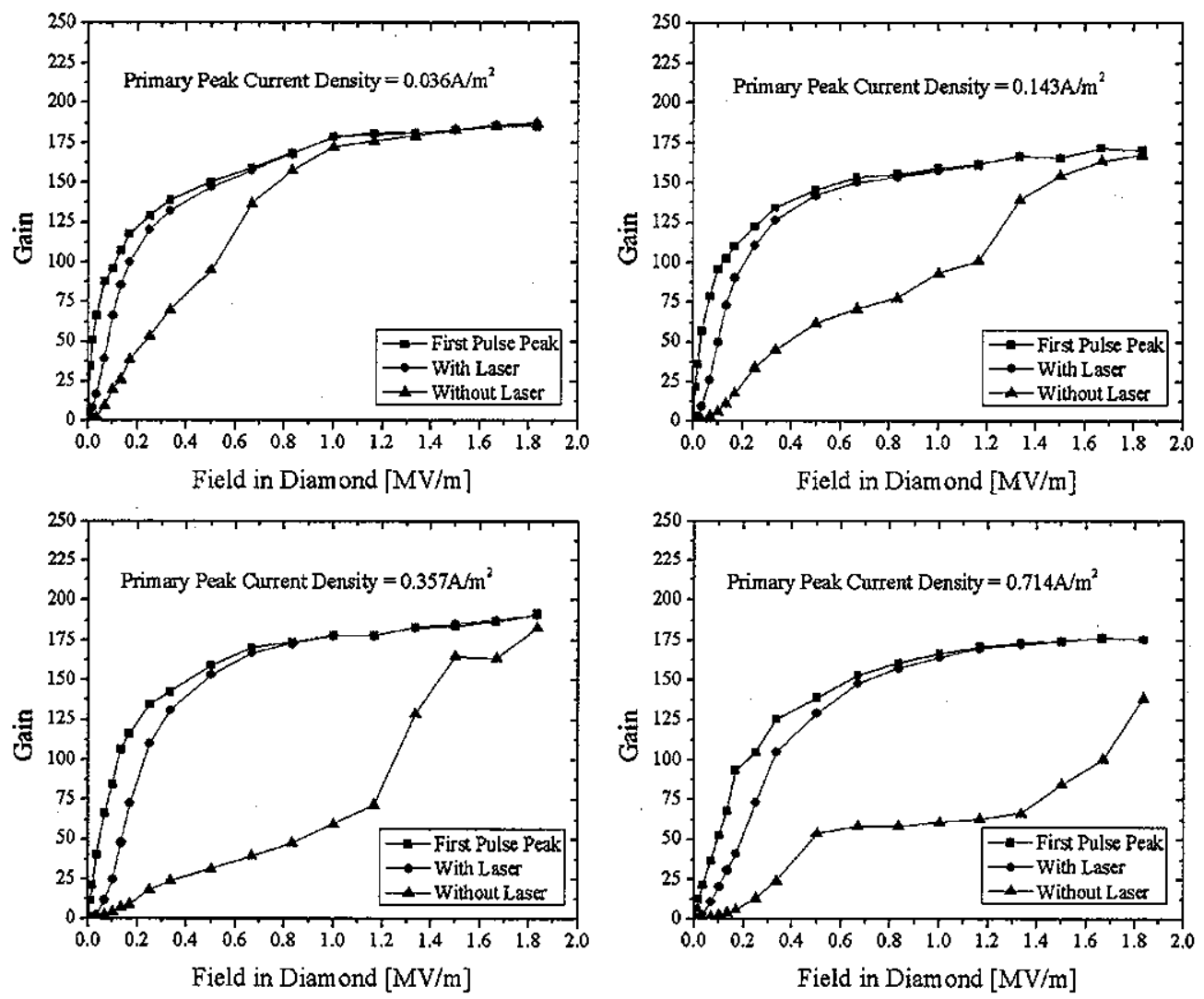

Figure 5.10: Gain comparison of with and without laser detrapping under different current densities. Primary electron energy fixed at $6 \mathrm{keV}$.

The transverse distribution of the primary beam is near uniform, and the diameter is fixed at $3 \mathrm{~mm}$. So the highest current density of the 4 cases should be $5 \mu \mathrm{A} /\left(\mathrm{p} \times 2.25 \mathrm{~mm}^{2}\right) \sim 0.7 \mathrm{~A} / \mathrm{m}^{2}$. The secondary electron current density is the product of the primary current density and the gain. With the same primary electron energy, the maximum gain under specific applied field should be the same.

Figure 5.10 shows the significant effect of laser detrapping in all 4 different 
primary currents, or current densities since the spot sizes for all case are the same. The gain curve with laser detrapping is very close to the first pulse gain curve, which is the maximum limit, even under a very small field and very high current density. Compared with the maximum, the gain obtained without laser detrapping shows a large decrease in value under low field and even under high fields when primary current density is high. Saturation of the gain curve can be reached without laser detrapping, but it would cost for a much higher applied field.

\subsubsection{Primary Energy}

Primary electron energy determines the saturated gain as discussed in Section 5.2 With the current density fixed this time, but changing the primary electron energy from $5 \mathrm{keV}$ to $8 \mathrm{keV}$, and the secondary electron current density changes correspondingly. The laser still brings the equilibrium gain close to the maximum gain at each applied field.

To reach equilibrium state, the set up without laser detrapping requires a much longer time for each data point. In the measurements, gain curves without laser were only taken for $6 \mathrm{keV}$ and $7 \mathrm{keV} .5 \mathrm{keV}$ and $8 \mathrm{keV}$ still show the same trend. 

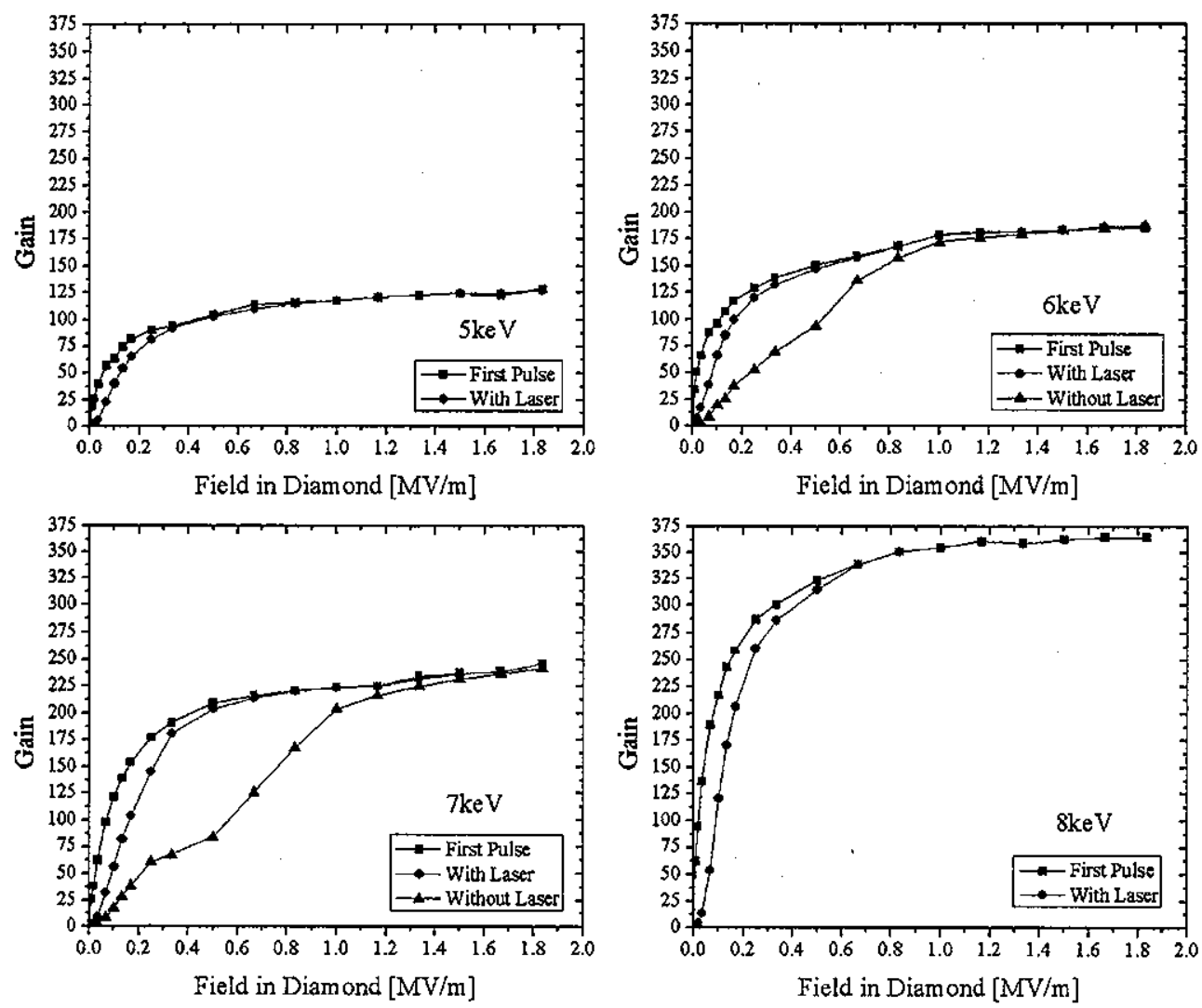

Figure 5.11: Gain comparison of with and without laser detrapping under different primary electron energies. The primary current density is fixed at $0.04 \mathrm{~A} / \mathrm{m}^{2}$.

The maximum gain increases with the primary electron energy, and so does the secondary electron current. As shown in Figure 5.11, without laser detrapping the gain requires about $1 \mathrm{MV} / \mathrm{m}$ of applied field to saturate in the $6 \mathrm{keV}$ case and even more in $7 \mathrm{keV}$ case, but with the laser, it only requires $<0.5 \mathrm{MV} / \mathrm{m}$. The gain w ith laser is much closer to the expected value than the gain without laser before reaching saturation. 


\subsubsection{Repetition Rate}

If the secondary current density is the same by fixing the primary electron energy and primary peak current density, the gain under low field will be determined by the time lapse between pulses. For a more comprehensive study compared to Figure 5.8 and Figure 5.9, Figure 5.12 below shows the laser effect on gain under different duty cycles. 

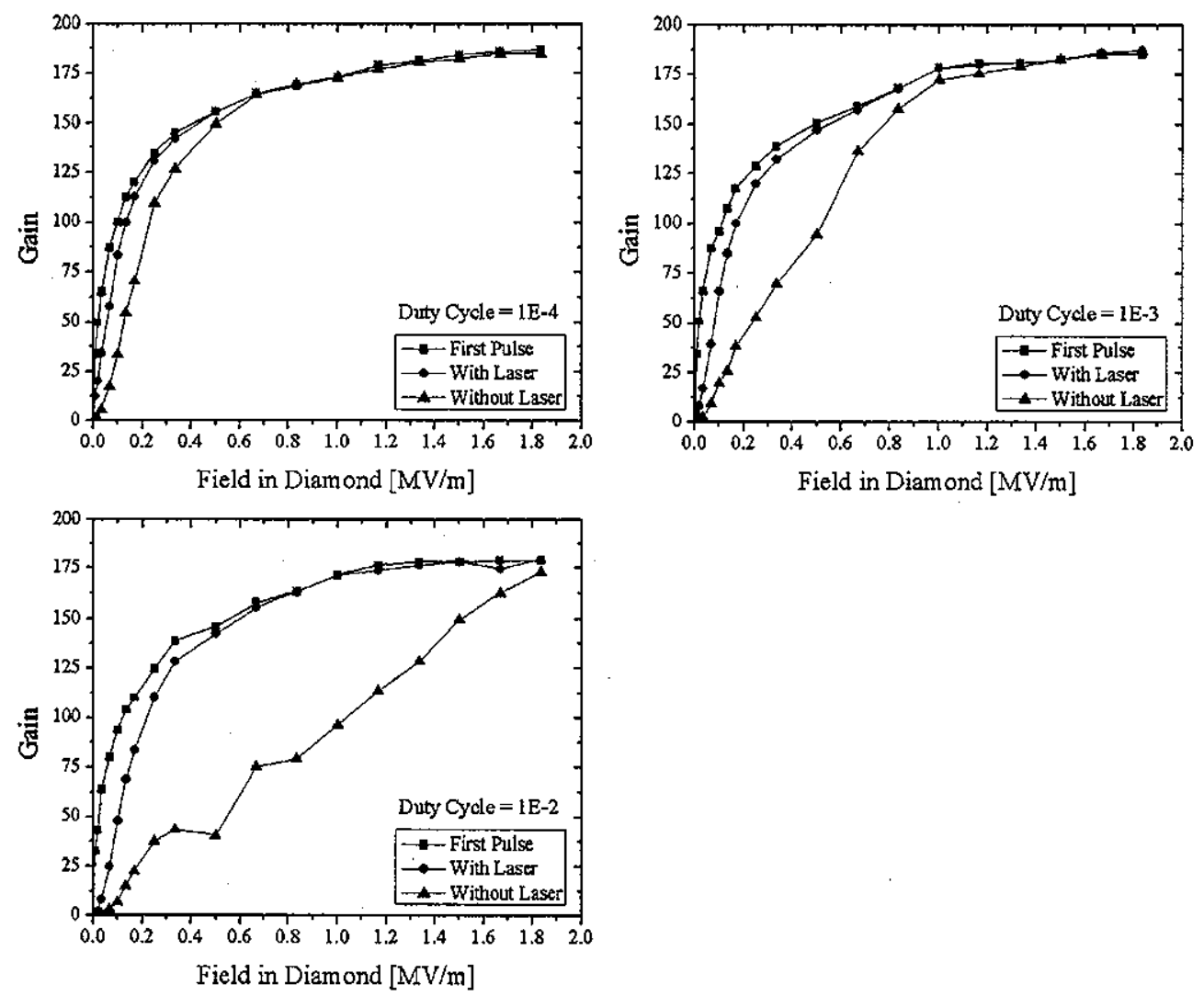

Figure 5.12: Gain comparison of with and without laser detrapping under various pulse repetition rates. The pulse width for each measurement is fixed at $10 \mu \mathrm{s}$.

Primary current density fixed at $0.036 \mathrm{~A} / \mathrm{m}^{2}$ and energy fixed at $6 \mathrm{keV}$.

Laser detrapping is essential to maintain a high gain under high repetition rates.

In the experiment for the measurement above, the pulse width is kept at $10 \mu \mathrm{s}$ continuously. Without the laser, the decrease in signal is rather trivial for the duty cycle of $1 E-4$. However, it became noticeable for duty cycle of $1 \mathrm{E}-3$, and unacceptable for $1 \mathrm{E}-2$. For a duty cycle of $1 \mathrm{E}-2$, the repetition rate would be $1000 \mathrm{~Hz}$, 
which is still very low compared with the frequency in most operating electron guns.

Laser detrapping shows significant increase in gain in transmission mode measurements. With the correct selection of wavelength, the trapped electrons can be released by photon-electron interaction. The laser used for detrapping is $523 \mathrm{~nm}$ in wavelength, which is corresponding to $2.34 \mathrm{eV}$. This energy is less than half of the band gap of diamond, which is $\sim 5.7 \mathrm{eV}$, and this ensures that all secondary electrons are created by the primary electrons rather than by photons. Other wavelengths have not been test quantitatively yet.

5.4 Energy lost in metal coating and estimation of energy needed for each $\mathrm{e}$ h pair

Primary electrons lose their partial energy in the metal coating, and the amount lost depends on the metal variety and thickness. Only the remaining energy contributes to the value of maximum gain in all curves in Figure 5.7.

According to $\operatorname{Ref}[24,25]$, the energy needed to create one e-h pair is fixed. The saturated gain should be a linear function of the primary energy. The intercept on horizontal axis in Figure 5.13 should be the energy lost in the metal coating $E_{\text {lost. }}$ The data of the maximum gain is averaged over data from $1 \mathrm{MV} / \mathrm{m}$ to $2.33 \mathrm{MV} / \mathrm{m}$. For a coating of $\sim 50 \mathrm{~mm}$ Pt on top of $\sim 30 \mathrm{~nm} \mathrm{Ti}$, the energy lost is $\mathrm{E}_{\text {lost }}=3.29 \pm 0.15 \mathrm{keV}$.

From the linear model approximation of the incident electron energy loss [26], the stopping powers for electron energy of ranged from $4 \mathrm{keV}$ to $8 \mathrm{keV}$ for $\mathrm{Pt}$ and $\mathrm{Ti}$ 
are:

$$
\frac{\Delta E}{\rho_{P t} \Delta x_{P t}} \approx 12 \mathrm{MeV} \cdot \mathrm{cm}^{2} / \mathrm{g} \quad \frac{\Delta E}{\rho_{\pi i} \Delta x_{T i}} \approx 20 \mathrm{MeV} \cdot \mathrm{cm}^{2} / \mathrm{g}
$$

where $?_{P}=21.45 \mathrm{~g} / \mathrm{cm}^{3}$ is the density of $\mathrm{Pt}$ and $?_{T l}=4.506 \mathrm{~g} / \mathrm{cm}^{3}$ is the density of $\mathrm{Ti}$, and $? x_{P t} \tilde{5} 50 \mathrm{~nm}$ and $? x_{\pi} \tilde{i} 30 \mathrm{~nm}$ are the thickness of the two layers of metal respectively. Then the total energy loss in the metal coating calculated from this model is:

$$
\Delta E_{\text {total }}=\Delta E_{P t}+\Delta E_{T i} \approx 1.556 \mathrm{keV} \text {. }
$$

The discrepancy between the linear model and the transmission measurement result is over $100 \%$, which is very big. This is due to the thickness measurement of the metal coating is not accurate, and should be verified.

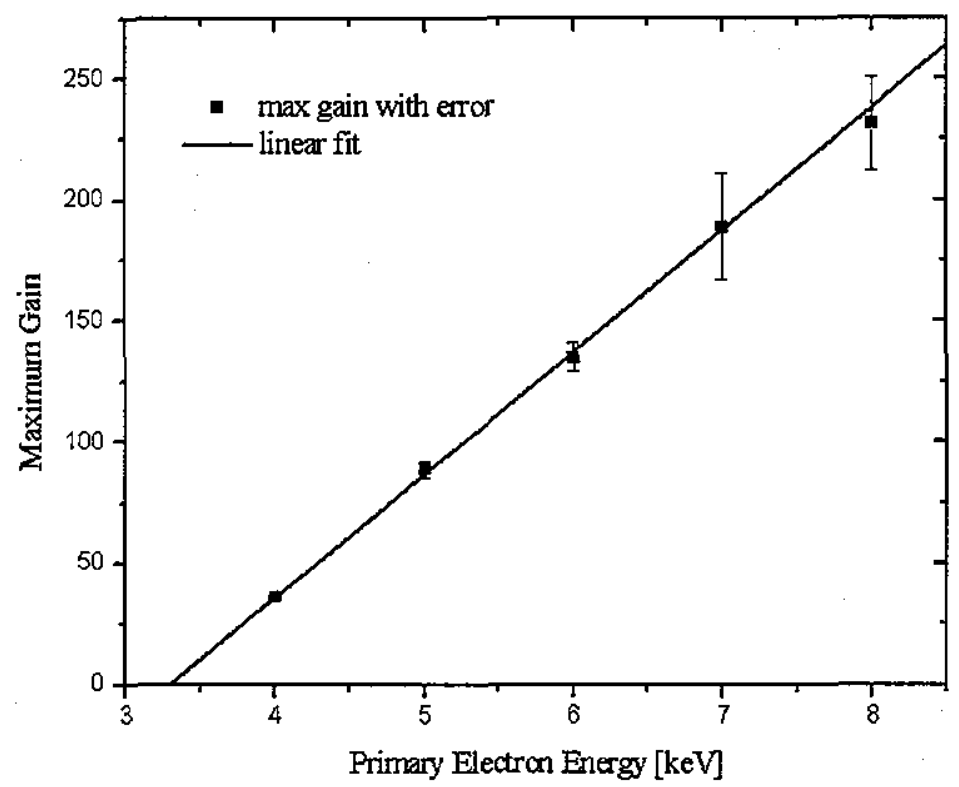

Figure 5.13: Maximum gain of different primary electron ener gy. 
The energy needed to create one e- $h$ pair is:

$$
\mathrm{E}_{e-h}=\frac{\text { primaryelectronenergy-energylostinmetal }}{\text { maximumgain }}
$$

Thus:

Table 5.1: Energy needed for create one electron hole pair.

\begin{tabular}{|c|c|}
\hline Primary Energy [keV] & Energy [eV] \\
\hline 4 & $19.80 \pm 1.29$ \\
\hline 5 & $19.25 \pm 1.61$ \\
\hline 6 & $20.06 \pm 1.82$ \\
\hline 7 & $19.63 \pm 3.67$ \\
\hline 8 & $20.33 \pm 2.60$ \\
\hline
\end{tabular}

The average is $19.82 \pm 4.93 \mathrm{eV}$. Former simulation approaches reported smaller values of $14.66 \mathrm{eV}$ and $17 \mathrm{eV}[24,25]$. The difference is possibly due simplification of the models and system error of the experiment.

\subsection{Recombination of the charges in diamond}

Diamond is an indirect band gap material, and its electron band structure has been published in 1966 by W. Saslow et. al. as shown in Figure 5.14 [27]. 


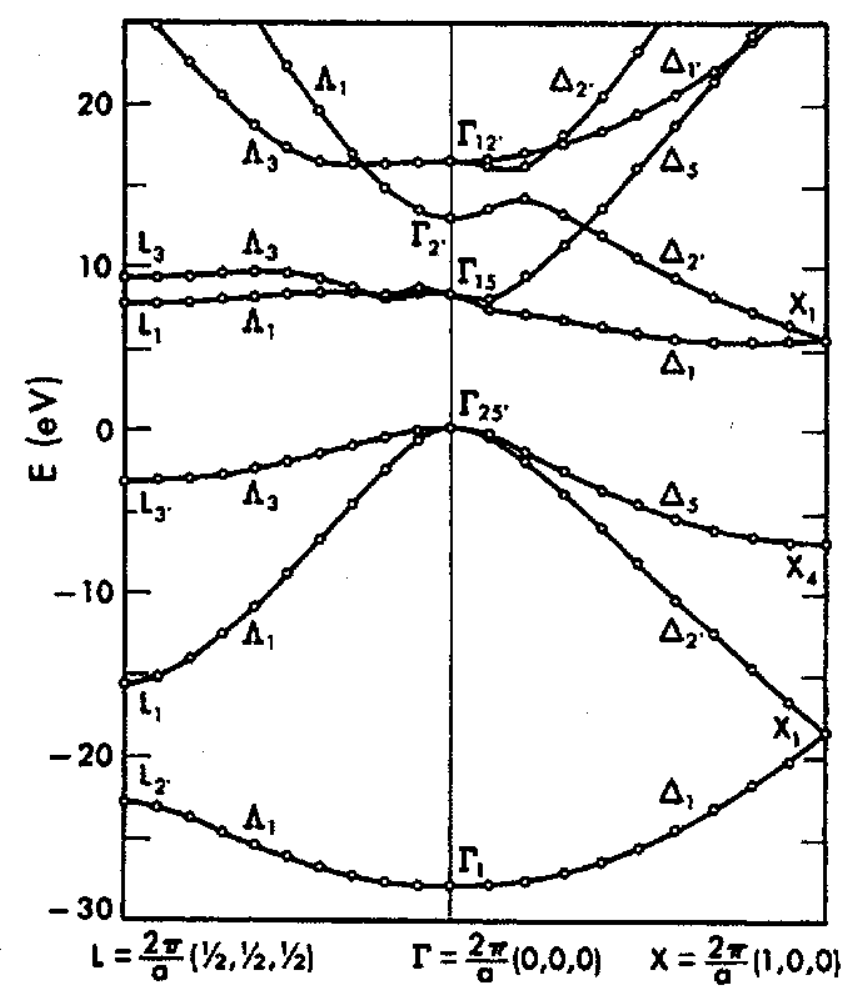

Figure 5.14: Diamond electron band structure.

Figure 5.14 shows a calculated band structure of the diamond which agrees with experiment. The figure covered a primitive cell of the reciprocal lattice in the frequency domain, or Brillouin zone, and each line indicates a different energy band. The vertical axis is the energy of the electron with $0 \mathrm{eV}$ as the Fermi level in diamond, and the horizontal axis is the value of wave vector $k$ in the reciprocal lattice. The zone center is $\mathrm{G} L$ and $X$ are boundary points at $(2 \mathrm{p} / \mathrm{a})(1 / 2,1 / 2,1 / 2)$ and $(2 \mathrm{p} / \mathrm{a})(100) . ?$ and ? are two axes along GL and GX directions respectively. The subscript labeled the number of energy band. 
The band gap of the diamond is:

$$
\begin{aligned}
& \mathrm{G}_{25^{\prime}}-?_{1}=5.4 \mathrm{eV} \quad \text { minimum value. } \\
& \mathrm{G}_{25^{\prime}}-\mathrm{G}_{15}=7.3 \mathrm{eV}
\end{aligned}
$$

Electrons present in the conduction band quickly settle into the energy minimum of that band. For indirect band gap materials, electrons in this minimum cannot rejoin the valence band by simply losing energy, or radiative recombination. They require some source of momentum allowing them to observe both the laws of conservation of energy and of momentum and fall into the valence band. The only way to promote this recombination is to simultaneously emit (or absorb) a phonon that compensates for the missing momentum vector, or a three-body collision with the impurities in the diamond. However, such a combined transition has a very low probability.

Another possible path for the secondary electrons to recombine is to move to the metal layer by diffusion. The secondary electrons and holes diffuse under Brownian motion once they are created. Any electron or hole crossing the diamond-metal interface will be recombined immediately in the metal layer and the defect rich diamond near the metal boundary. Given that the plasma is formed tangent to the interface and all charge particles are moving with random walks, the electrons and holes are merged into the metal continuously through this diffusion process.

To illuminate the process, let us apply some numbers. Assume the SEY of the primary electron is 100 , and initial RMS radius of the plasma after thermal relaxation is $10 \mathrm{~nm}$. The density of the charged cloud under this assumption would be: 


$$
n_{e}(t=0)=n_{h}(t=0)=n_{0}=2.4 \times 10^{25} / \mathrm{m}^{3}
$$

The Debye radius of this cloud should be:

$$
\lambda_{D}=\left(\frac{\varepsilon k_{B} T}{q^{2} N_{0}}\right)^{1 / 2} \approx 4.1 \times 10^{-10} \mathrm{~m}=0.41 \mathrm{~nm}
$$

under room temperature, which is more than 20 times smaller than the dimension of the cloud. Therefore the charges can be considered as plasma at the initial state.

The plasma frequencies for both electron and hole clouds are:

$$
\omega_{p, e}=\frac{n_{0} e^{2}}{\varepsilon_{0} m_{e}} \quad \text { and } \quad \omega_{p, h}=\frac{n_{0} e^{2}}{\varepsilon_{0} m_{h}}
$$

where $m_{e}$ and $m_{h}$ are effective masses of electron and hole inside the diamond. The mobility of the two charge carriers are comparable and both are in the $10^{5} \mathrm{~m} / \mathrm{s}$ scale $[29,30]$, therefore it is reasonable to assume the effective masses are similar and both close to the rest mass of electron. Plug in the values for the equation above, the plasma frequencies should both be $\sim 2.8 \times 10^{14} \mathrm{~Hz}$.

For applied DC (or slowly varying) field, the attenuation constant is:

$$
\alpha_{\text {plasma }}^{-1} \approx \frac{c}{2 \omega_{p}} \approx 540 \mathrm{~nm}
$$

which is much larger than the RMS radius of the plasma.

This indicates that the applied field penetrates through the full length of the electron and hole clouds at the initial state without much decrease in the amplitude. The plasma would start to separate under applied electric field. 
As the electric field pulls the electron cloud away from the interface to prevent loss of the electrons, the diffusion proess, on the contrary, will keep pushing electrons into the metal to be recombined. The eventual gain measured in the experiment is the compromise of the two motions. High electric field can accelerate the electrons to their saturation velocity away from the incident surface within a short period of time, so that fewer electrons are lost. Therefore, the gain is small under low electric field, and increases rapidly with the field amplitude until the drift velocity of the electrons can reach the saturation velocity. The gain will not change as much after the electron drift velocity saturates within a short distance. This explains the gain curve shape in Figure 5.7. 


\section{EMISSION MODE MEASUREMENT OF DIAMOND SECONDARY ELECTRON Y IELD}

\subsection{Measurement setup}

The emission mode measurement system reproduces the setup of the transmission mode measurement discussed in Chapter 5, with the exception that we introduced a vacuum gap between the copper anode and the diamond surface, and the termination of this surface is changed to hydrogen instead of a metal coating.

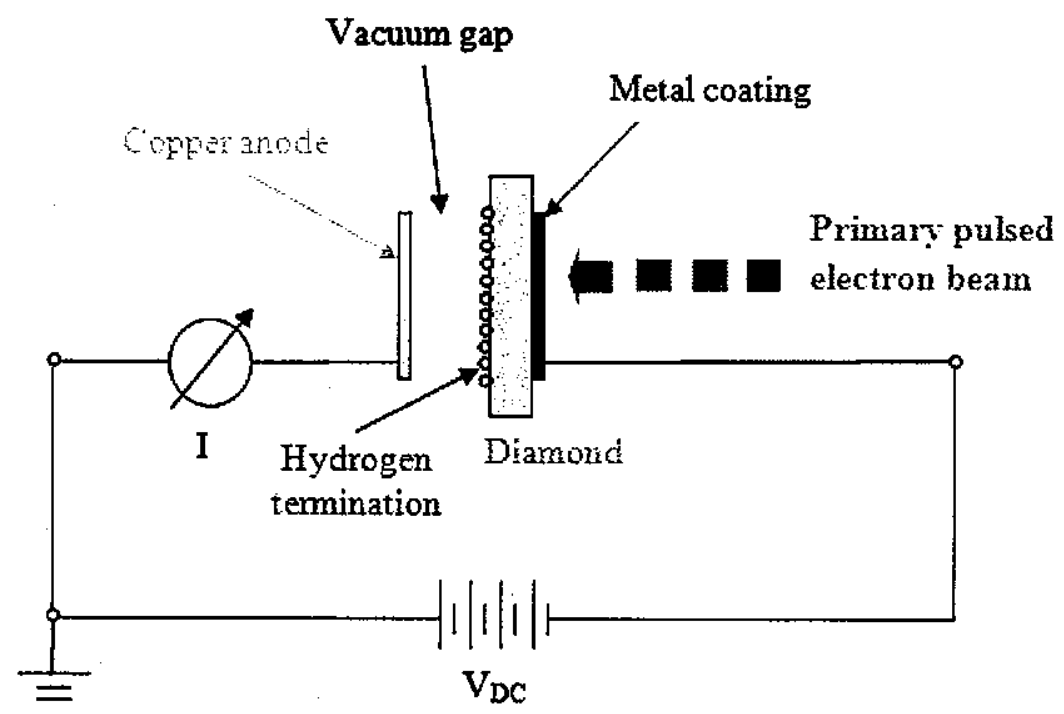

Figure 6.1: Simple sketch of emission mode measurement principle. Either side can be grounded.

With hydrogen termination, the NEA surface will allow the secondary electrons 
to escape into vacuum and get collected by the anode. An amplifier circuit was designed and added for signal collection. With this improvement; the response time decreased from $\sim 1 \mu$ s into $\sim 20 \mathrm{~ns}$.

The emitted electrons can be measured by an oscilloscope connected to either the anode or cathode. In the anode connection measurements, the anode is grounded and the metal coating on the incident side is negatively biased. The effective energy of the primary electrons contributed to the emission is calculated as

$$
E_{\text {effective }}=E_{\text {primary }}-U_{\text {metal }}-E_{\text {lost }} \text {, }
$$

where $E_{\text {primary }}$ is the primary electron energy at the exit of the gun, $U_{\text {metal }}$ is the negative bias voltage on the metal coating, and $E_{\text {lost }}$ is the energy lost in the metal coating.

The grounding of the anode is designed for application of a phosphor screen and a CCD camera. If a focusing device is applied between the anode and the phosphor screen and the distance is carefully chosen, the thermal emittance of the diamond amplifier can be measured by imaging the beam spot size on the CCD camera. The design for this measurement will be discussed in Chapter 7 .

\subsection{DC beam or continuous long pulse measurements}

The trapping issue in emission mode measurement is more problematic due to the critical process of hydrogenation. For the hydrogenation process, the diamond is 
heated to above $800^{\circ} \mathrm{C}$ to break the carbon-oxygen bond and leave free dangling carbon bond on the surface. Hydrogen gas s broken into atoms (or "cracked") and impacts the diamond surface. We use a commercial thermal gas cracker (Mantis MGC-75) to carry out this process which leads to create negative affinity surface (NEA) on the diamond. During the formation of carbon-hydrogen bonds, it is likely to have residual dangling carbon bonds on the surface without any hydrogen attached. The dangling bonds are capable of trapping the secondary electrons transferred to the hydrogenated surface. The field applied to the diamond during experiments is about $1 \mathrm{MV} / \mathrm{m}$, and to cancel such field, the trapped electron density only needs to be $0.5 \mathrm{C} / \mathrm{cm}^{2}$, which is about 1 trapped electron per 10,000 conventional unit cells on the surface. This is a very small number considering the occupation of hydrogen coverage during CVD diamond [111] surface growth can be as low as $86 \%$ [33].

With a continuous primary electron beam, sufficient time is allowed for all possible trapping events to occur. The signal measured on the anode side comprises the trapping and releasing of the electrons in the diamond. The signal detected is purely caused by emitted electrons and has no capacitive coupling components. 


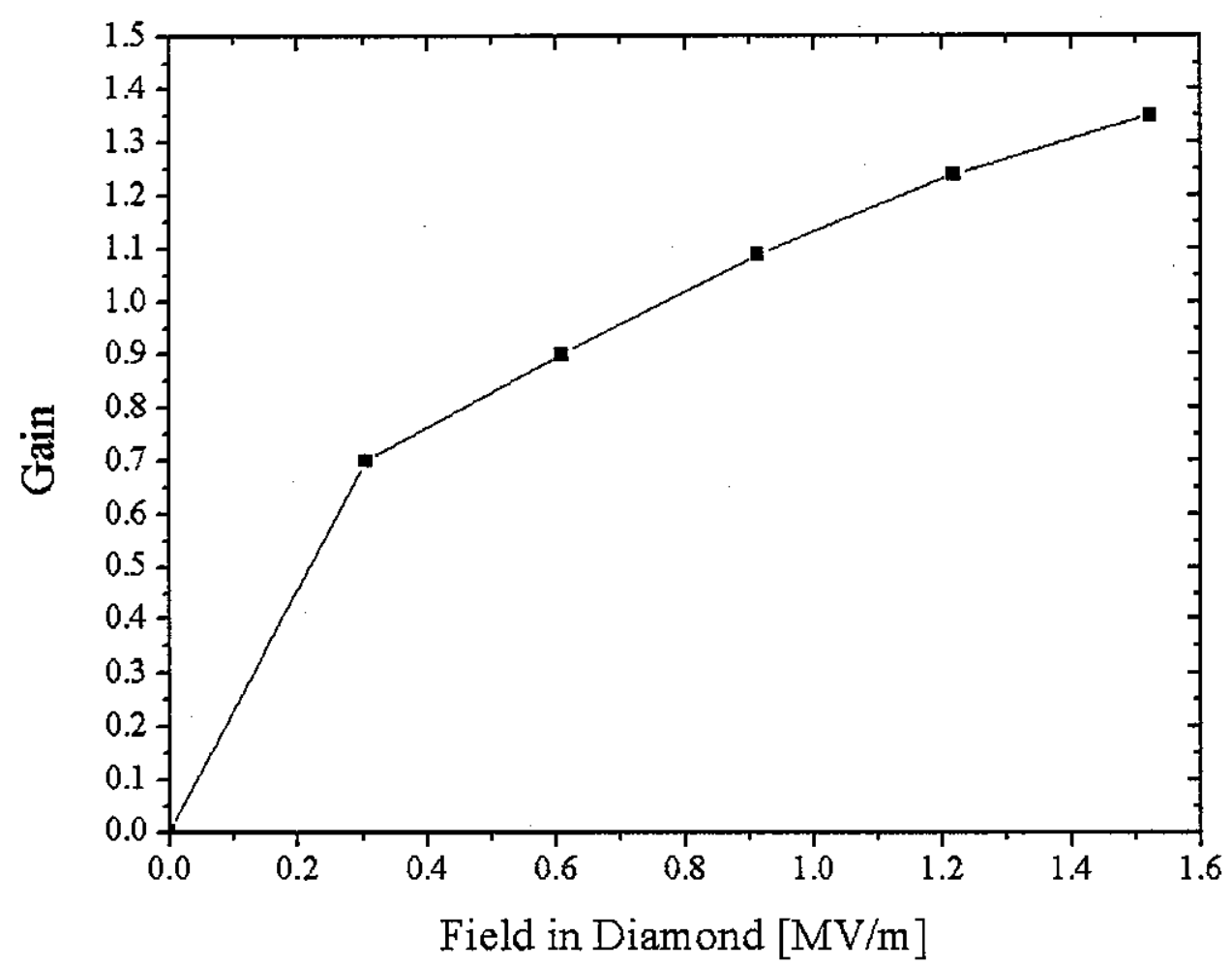

Figure 6.2: Emission mode gain with DC primary beam.

The signal is much smaller than the transmission mode measurements, and green laser had no effect on the signal. Since the dangling bond state energy is more than $1.3 \mathrm{eV}[34,35]$, the green laser detrapping is not as effective in emission mode, because the electrons are trapped much deeper by dangling bonds on the surface than the trapping under the surface caused by impurities and stress in lattice. The signal will increase with better hydrogenation, which will reduce the number of dangling bonds on the surface. 


\subsection{Short-pulse measurements}

Despite the small signal in the DC beam mode, the diamond performance is significantly better in shortpulse measurements. As in transmission mode measurements, electrons in the beginning of the first pulse applied to the diamond will experience a strong, unshielded applied field, and have much higher possibility to emit. The gain in the beginning of the pulse is much higher than the value measured under equilibrium state.

In real applications, the applied pulse would be very short. As an example, the proposed ecooling facility in Brookhaven National Laboratory has a frequency of $703 \mathrm{MHz}$ The pulse from the source must be much shorter than 1 s. Therefore the performance of the diamond amplifier with short pulses is more important and practical.

For short pulse measurements, the diamond was first set at neutral. Shortly after $(<3 \mathrm{~s})$ the electric field was applied, a train of pulses of $40 \mu$ s was sent to impact the diamond from the electron gun. With the fast response signal collection circuit, only the SEY of the first pulse was recorded. This ensures that the front segment of the pulse experienced the field as applied, and the change of the signal due to trapping and shielding of the field is reflected later in the pulse.

\subsubsection{Delay time dependence}

During pulse measurements, the diamond is first bombarded with continuous 
primary electrons and no electric field is applied. Any previously trapped charge will cause attract opposite species to transport through the diamond and recombine. This will 'clean up' the diamond electrically and achieve a neutral state after a very short time.

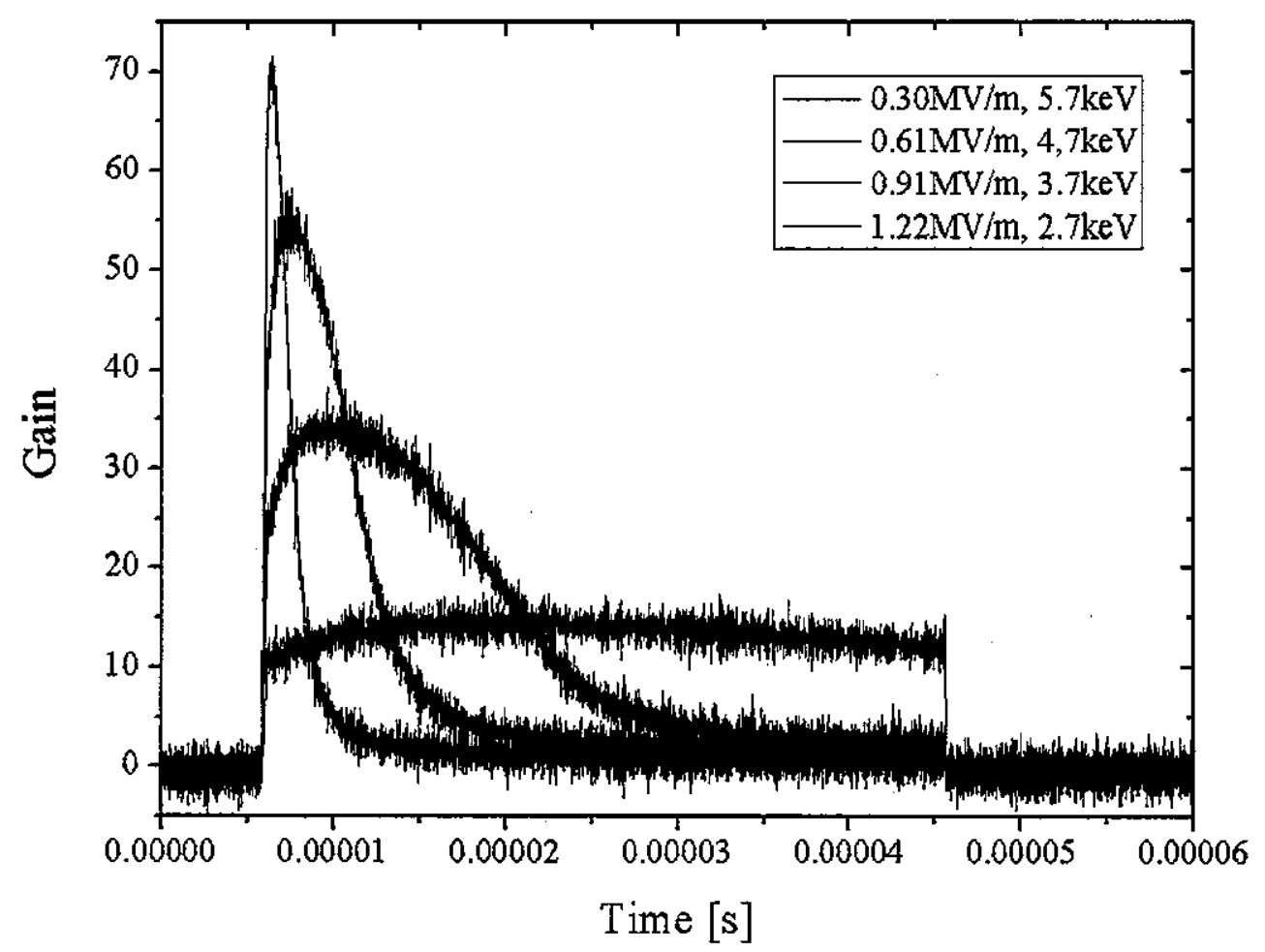

Figure 6.3: First pulse gain under room temperature $\left(23^{\circ} \mathrm{C}\right)$ with different field and effective energy for each pulse. Effective energy is the energy of the primary beam excluding the energy loss in the metal coating.

High purity single crystal diamond is a good insulator; however free charge-carriers inside the diamond that move according to the applied field, as in the 
application of diamond detectors. There are also few trap centers in the bulk to trap the charge carriers and therefore cause shielding to the applied field. In pulsed measurements, the time delay of the first pulse after applying the electrical field is critical to the amplitude of the gain.
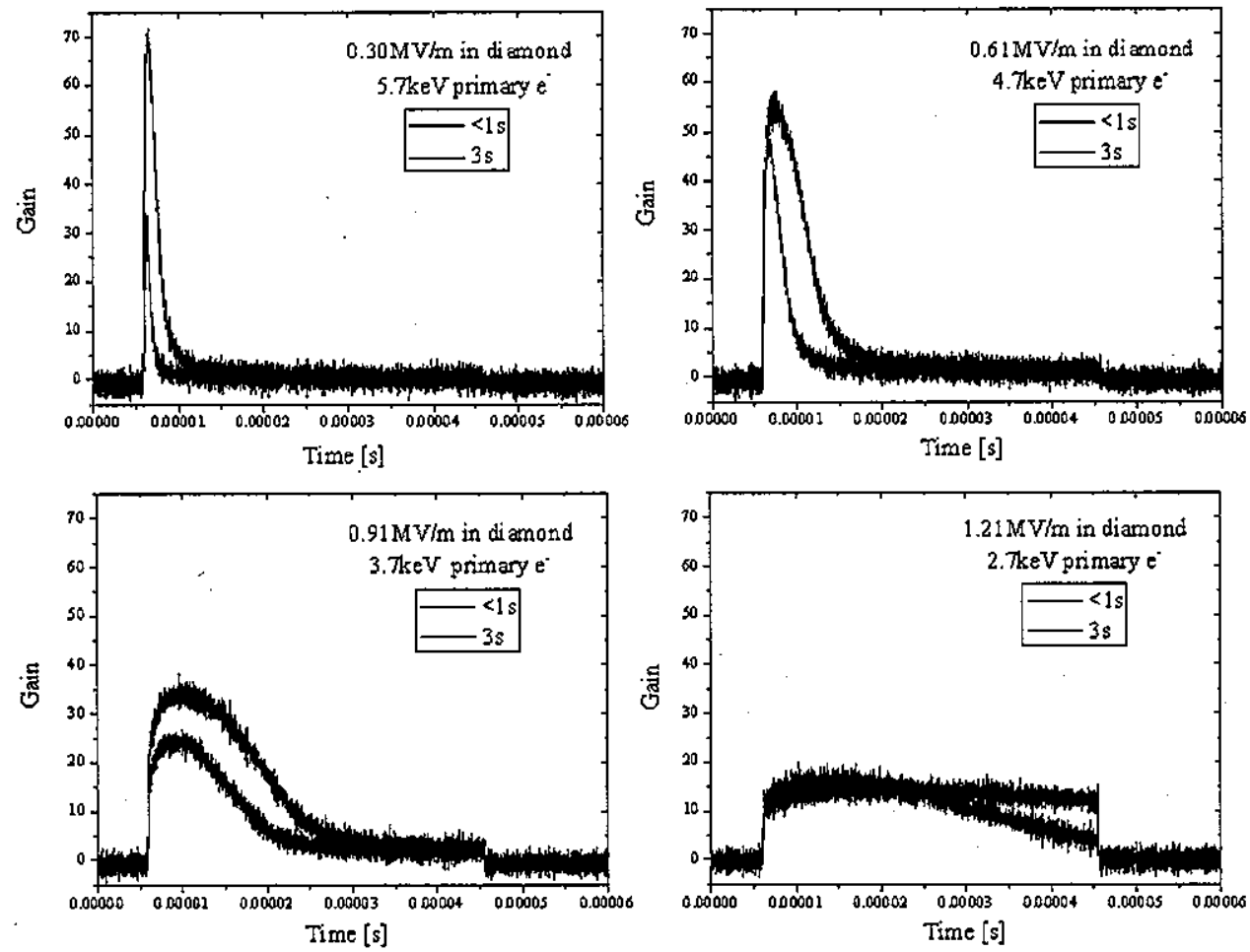

Figure 6.4: Secondary signal with different delays for the primary electron after applying the $\mathrm{DC}$ field.

Figure 6.4 shows the difference between two delay times, $<1 \mathrm{~s}$ and $3 \mathrm{~s}$. For all four applied field strengths, the first pulses with longer delay time have smaller gain 
at the beginning of the pulse and decreased under approximately the same rate with the shorter delay time pulses. The gain at the beginning of the pulse is mainly defined by the existing field in the diamond when the pulse is applied, and the decrease afterwards shows trapping by the diamond as discussed above.

Notice that the delay time effect is significant here on the scale of seconds, which is very long compared to the eventual use of the diamond amplifier. Thus the data above with long time scale is just to show the exaggerated difference, but this will probably not be an issue in the real application.

\subsubsection{Temperature dependence}

As the diamond samples are pure single crystal diamonds, the trap centers' density in the bulk is very small. This is demonstrated in the transmission measurements where the signals are much higher than that in the emission mode. There is another mechanism of trapping and possibly this is the dominant reason for the signal's decrease with time, that is the effect of the dangling bonds. The dangling bonds in emission measurements are serious obstacle for achieving a large gain. Before the primary electron would reach the diamond, the dangling bonds of the surface atom would capture electrons from nearby carbon atoms. This will generate a hole in the valence band. The hole moves to the cathode surface under the influence of the applied electric field. The time it takes the holes to traverse the thickness of the diamond is under one nanosecond, and this will lead to charging up of the emitting 
face and thus to a decrease of the field of the diamond. This will result in a gain decrease due to the density of the dangling bonds.

Nevertheless, the density of ionized dangling bonds is a function of temperature in materials, and this will be critical because the diamond amplifier will be operated at a low temperatures in actual applications [36].

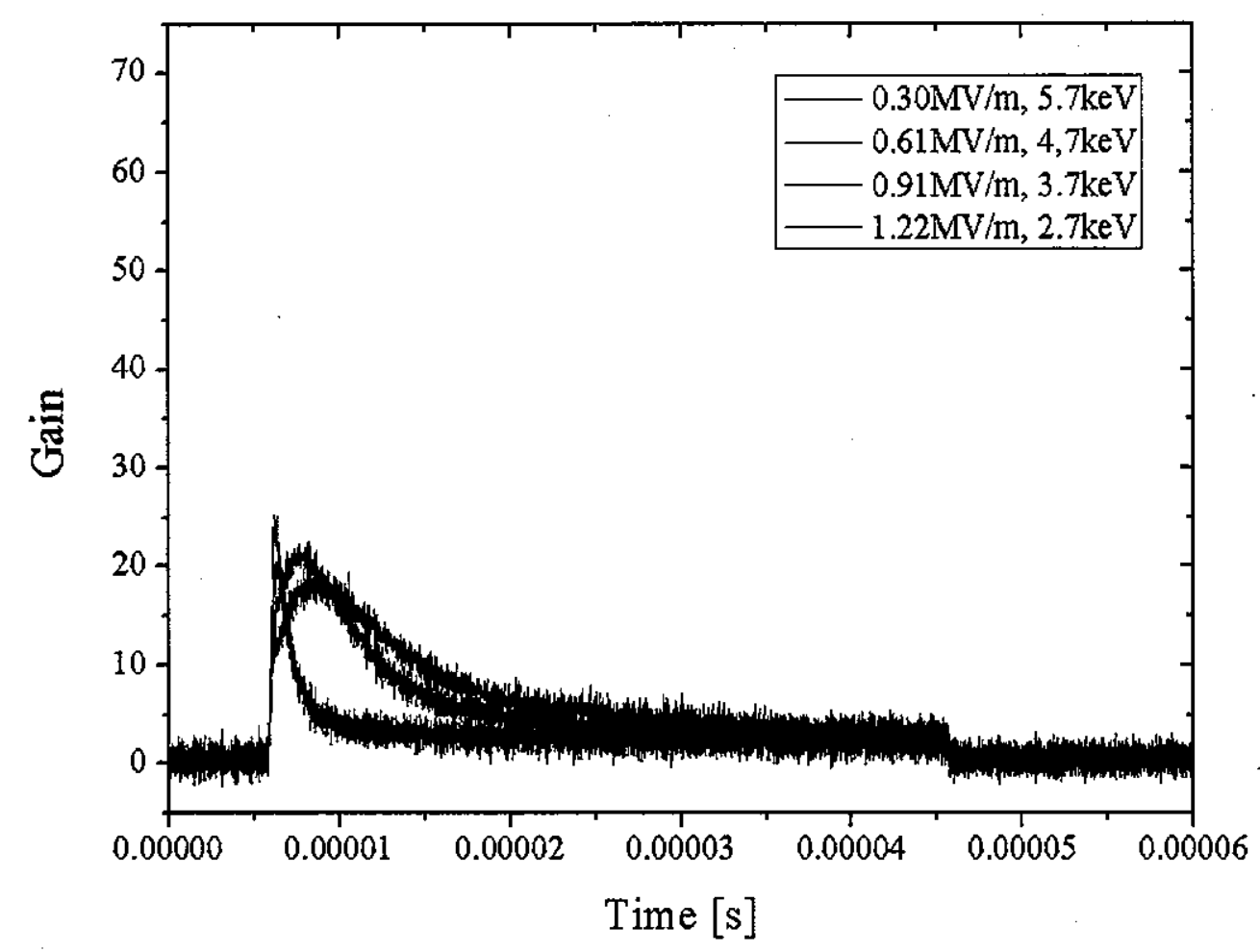

Figure 6.5: Emission signal of the diamond amplifier at $200^{\circ} \mathrm{C}$.

In Figure 6.5 , the diamond sample was heated to $250^{\circ} \mathrm{C}$ and kept under this specific temperature for more than 3 hours to remove the $\mathrm{H}_{2} \mathrm{O}$ molecules attached on the hydrogenated surface. The heating procedure leads to $\mathrm{H}_{\mathrm{O}} \mathrm{O}$ removal from the 
H-terminated surface of the CVD diamond as described in reference [37]. The NEA surface of the diamond should be improved after the heating cycle. To ensure the monolayer of hydrogen is not covered by atoms from the residual gas, the sample was reheated to $200^{\circ} \mathrm{C}$ and kept under such temperature during the experiment. Comparing with Figure 6.3, which shows room temperature $\left(23^{\circ} \mathrm{C}\right)$ results, the gain at the beginning of the pulse in the high temperature $\left(200^{\circ} \mathrm{C}\right)$ environment is much lower. Also in high temperature signals, the decrease of the gain is much faster than that decrease observed at room temperature. This indicates that the trapping effect on the surface of the diamond is more significant under higher temperature. Higher temperature with lower SEY is consistent with the dangling bonds density positive depende nce over the temperature.

Under the same conditions, liquid nitrogen cooled system should give higher gain than the above two cases. 


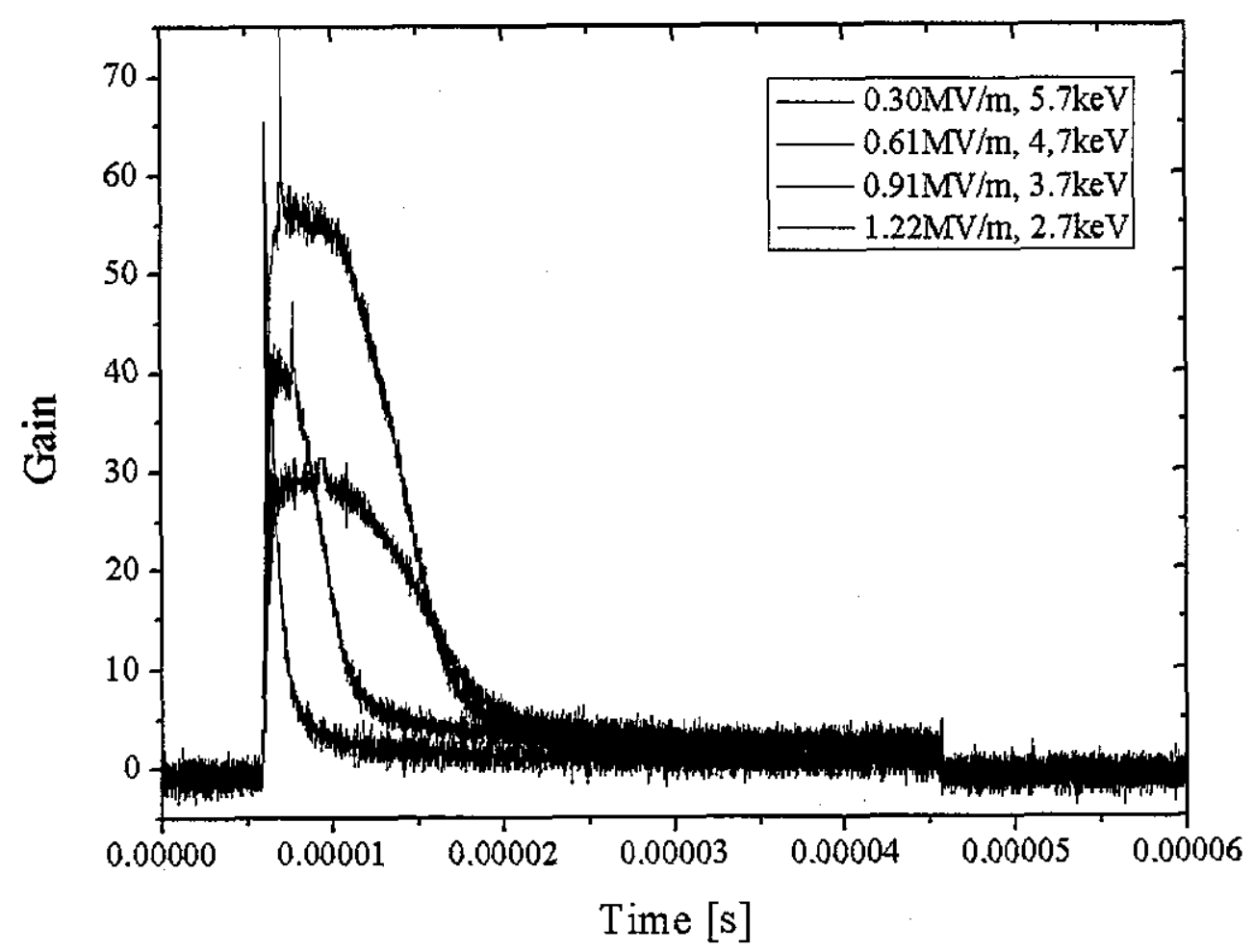

Figure 6.6: Emission signal of the diamond amplifier at $-140^{\circ} \mathrm{C}$.

For the gain results shown in Figure 6.6, the sample and holder are cooled by a liquid nitrogen cooling loop, and the temperature of the whole system is calibrated to be at $-140^{\circ} \mathrm{C}$. The gain in this case is higher than Figure 6.3 under high field. With high SEY, the system can still maintain the gain for a fairly long time. This indicates that the field does not decrease as fast as under higher temperatures, namely many more electrons are emitted within the pulse. Hence, to obtain the best performance of the diamond amplifier, it should be operated with the ambient temperature as low as possible. 
Thus with proper hydrogenation, the diamond amplifier will achieve reasonable gain $(>50)$ in nanosecond or shorter pulses under low temperature.

\subsubsection{Laser effect}

In transmission measurements, the laser helped improve the gain of the diamond. But under emission mode, the main concern comes from the dangling bond on the hydrogenated surface. The photon's energy is above the energy gap between the dangling bond and the top of the valence band. So the laser increases the ionization probability of the dangling bonds. The photons also will heat up the surface temperature by photon-electron interaction, and this will also increase the dangling bond ionization by a small degree.

Figure 6.7, Figure 6.8, and Figure 6.9 are three emission temperature measurements with laser applied and only temperature difference. All figures are plotted with the same scale for easier comparison. All three figures show much lower gain and faster falling edge than the corresponding temperature with no laser applied in Sections 6.3.1 and 6.3.2 Therefore trapping centers considered under transmission mode are less effective to the signal in emission mode, and photons create more dangling bond ionization. The shielding from the dangling bond ionization is more serious under emission. This is reasonable considering the high purity and careful surface treatment of the diamond samples. 


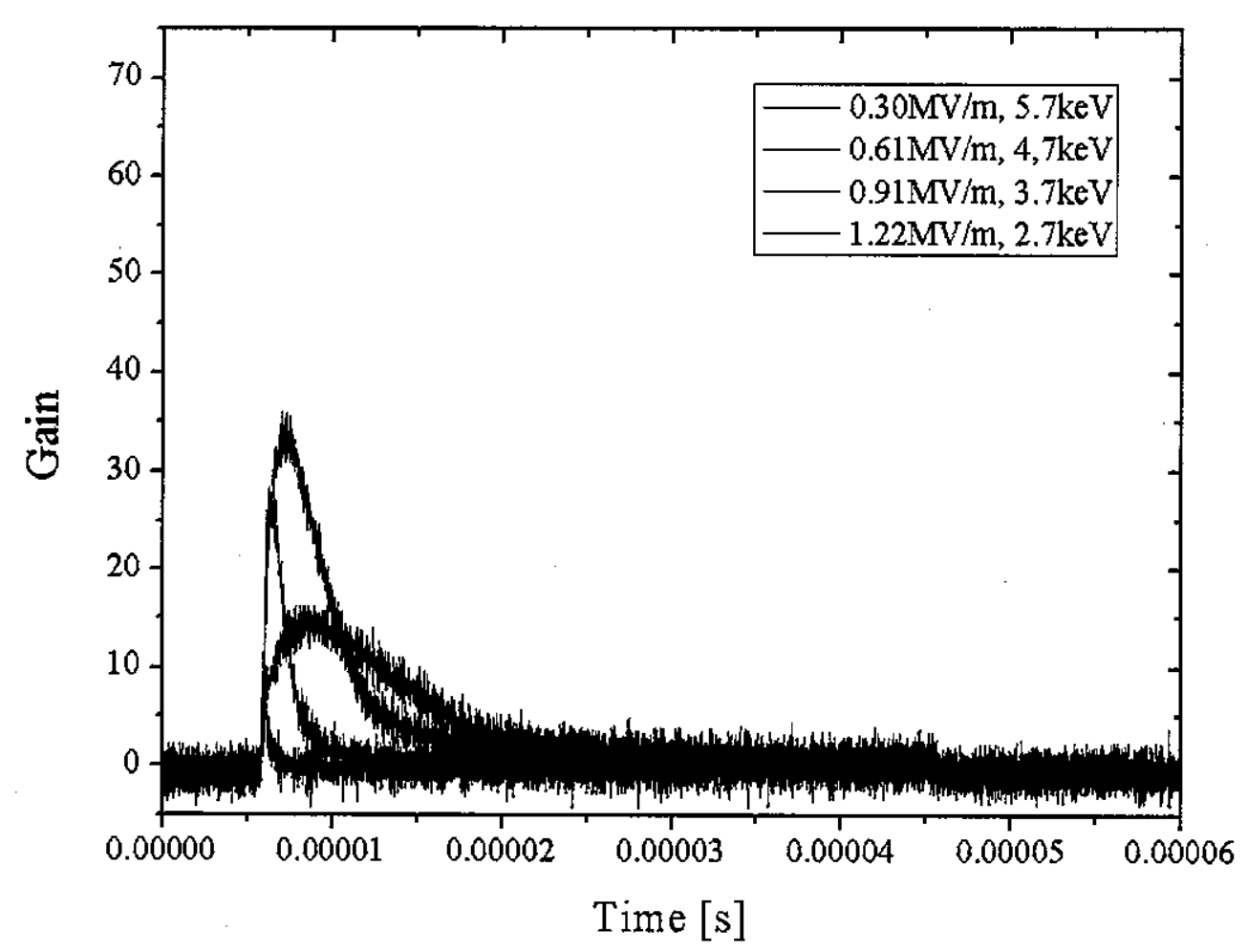

Figure 6.7: Room temperature $\left(23^{\circ} \mathrm{C}\right)$ measurement with laser applied $(532 \mathrm{~nm}$ laser, $<5 \mathrm{~mW})$. 


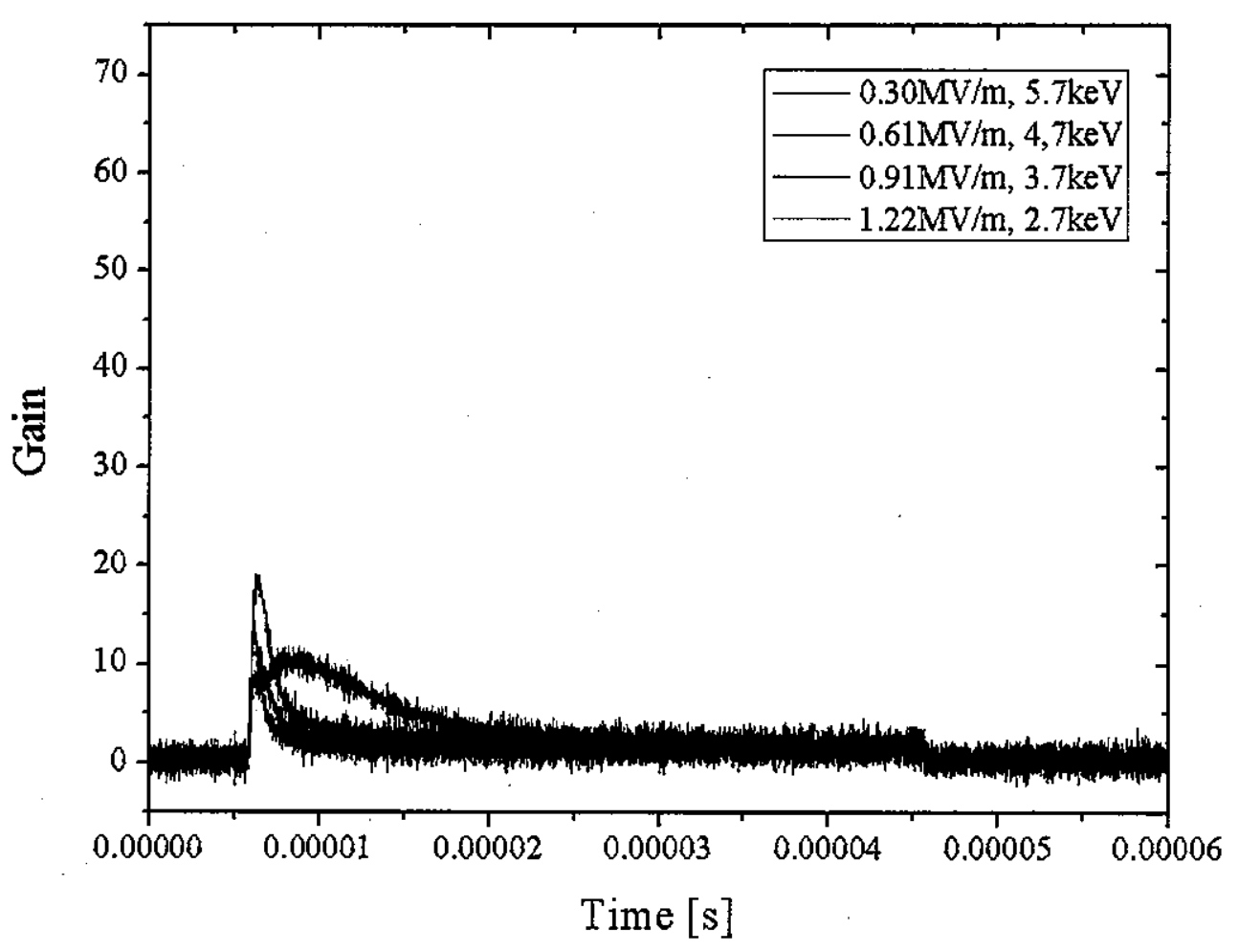

Figure 6.8: High temperature $\left(200^{\circ} \mathrm{C}\right)$ measurement with laser applied $(532 \mathrm{~nm}$ laser, $<5 \mathrm{~mW}$ ). 


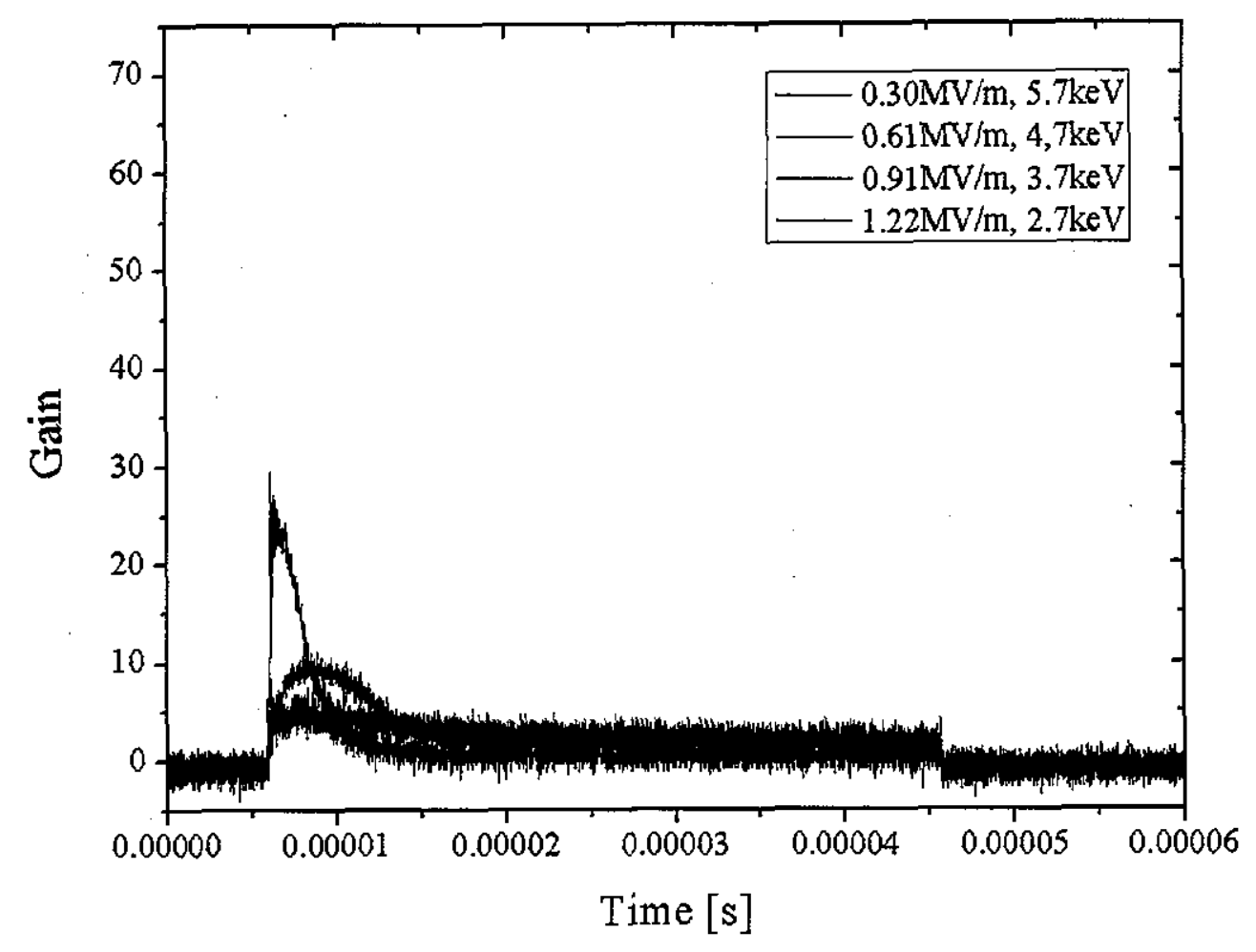

Figure 6.9: Low temperature $\left(-140^{\circ} \mathrm{C}\right)$ measurement with laser applied $(532 \mathrm{~nm}$ laser, $<5 \mathrm{~mW}$ ).

Focus ing on the shape and amplitude of the signal, it is noticeable that the higher the temperature, the less the difference between with and without laser results. This also proves the dangling bond charging density is highly dependent on the temperature. The thermal energy brought by photons is very small due to the low power of the laser. Hence under higher temperature, the dangling bond density is defined mainly by the heating source applied instead of the laser. 


\section{THERMAL EMITTANCE MEASUREMENT DESIGN}

The ultimate emittance of a laser-photocathode gun is limited by the thermal emittance. As laser shaping techniques improve, this limit is becoming more important. The thermal emittance of positive electron affinity photocathodes in terms of the thermal electron energy is a large fraction of an electron volt, depending on the type of photocathode. The diamond amplified photocathode, being a negative electron affinity (NEA) cathode, promises to deliver a very small thermal emittance. Theoretical expectations as well as measurements in the reflection mode point towards a temperature less than $0.1 \mathrm{eV}$. This has never been measured in a transmission mode. Therefore the aim is to measure the thermal emittance of hydrogenated NEA diamond.

The thermal energy of the secondary emission beam depends on the characteristic of the diamond sample, the properties of the hydrogenated surface and the electric field in the diamond, and yet it is expected that the thermal energy is not a strong function of the field. The measurement system should have the ability to measure this small emittance.

The design is shown in Figure 7.1. The beam coming out of the diamond NEA surface is accelerated towards an anode. The anode has a small hole, to collimate an output beam. The output beam is focused on a screen by a solenoid. The main idea of 
the measurement is to compensate the strong linear defocusing effect at the anode by applying a linear focusing lens and measure the waist spot size downstream. The waist spot size is only a function of the thermal emittance and is less affected by the other components. Therefore, we can compare the measurement with simulation [38]. It can also be calibrated with known thermal emittance electron source such as a thermal cathode.

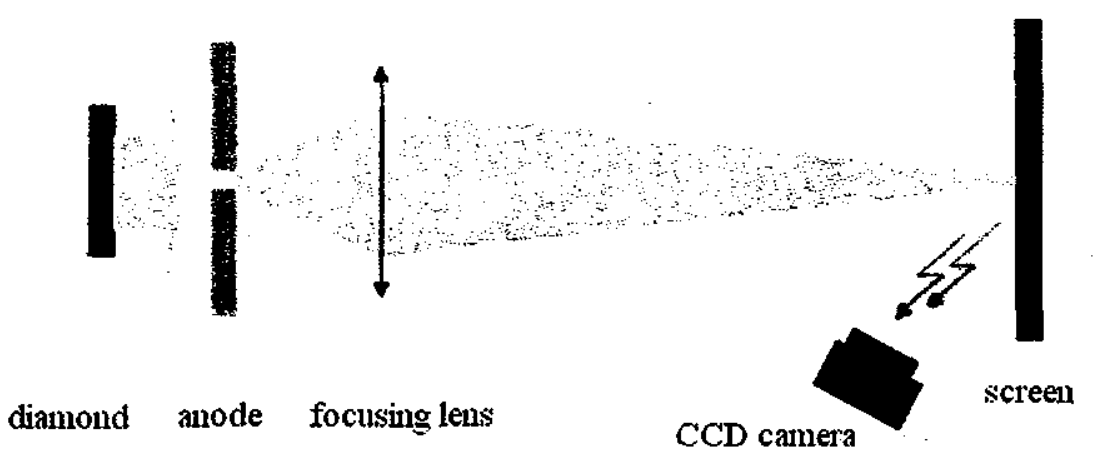

Figure 7.1: Setup design for thermal energy measurement of diamond secondary emission.

\subsection{Methods}

Common to all possible focusing schemes for thermal energy measurement is the application of a small aperture on the anode plate to limit the beam size, such that the beam size after a drift distance is measurable. In addition, the field applied for acceleration is constrained by the need to obtain a good gain in the diamond amplifier. 
The schematic layout of the cathode and anode is shown in Figure 7.2.

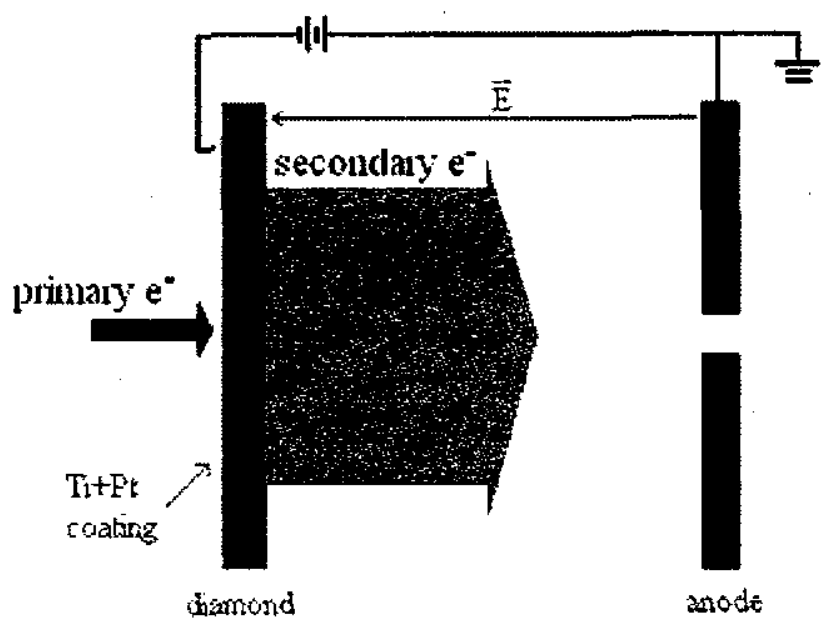

Figure 7.2: Acceleration of the secondary electrons by applying a high voltage across the diamond and anode.

\subsection{Limitations}

The aperture in the anode applies a defocusing force on the electron beam, which is mainly a linear force with a small component of nonlinear aberration. A larger aperture will lead to a stronger aberration, and will cause an increase in the beam spot size on the screen. Space charge is another issue in increasing the spot size. A small aperture in the anode also leads to a lower current with smaller space charge effect, increasing the accuracy of the measurement. On the other hand this will make the 
imaging more difficult. From simulation results in Figure 7.3, we choose the upper limit for each effect. 

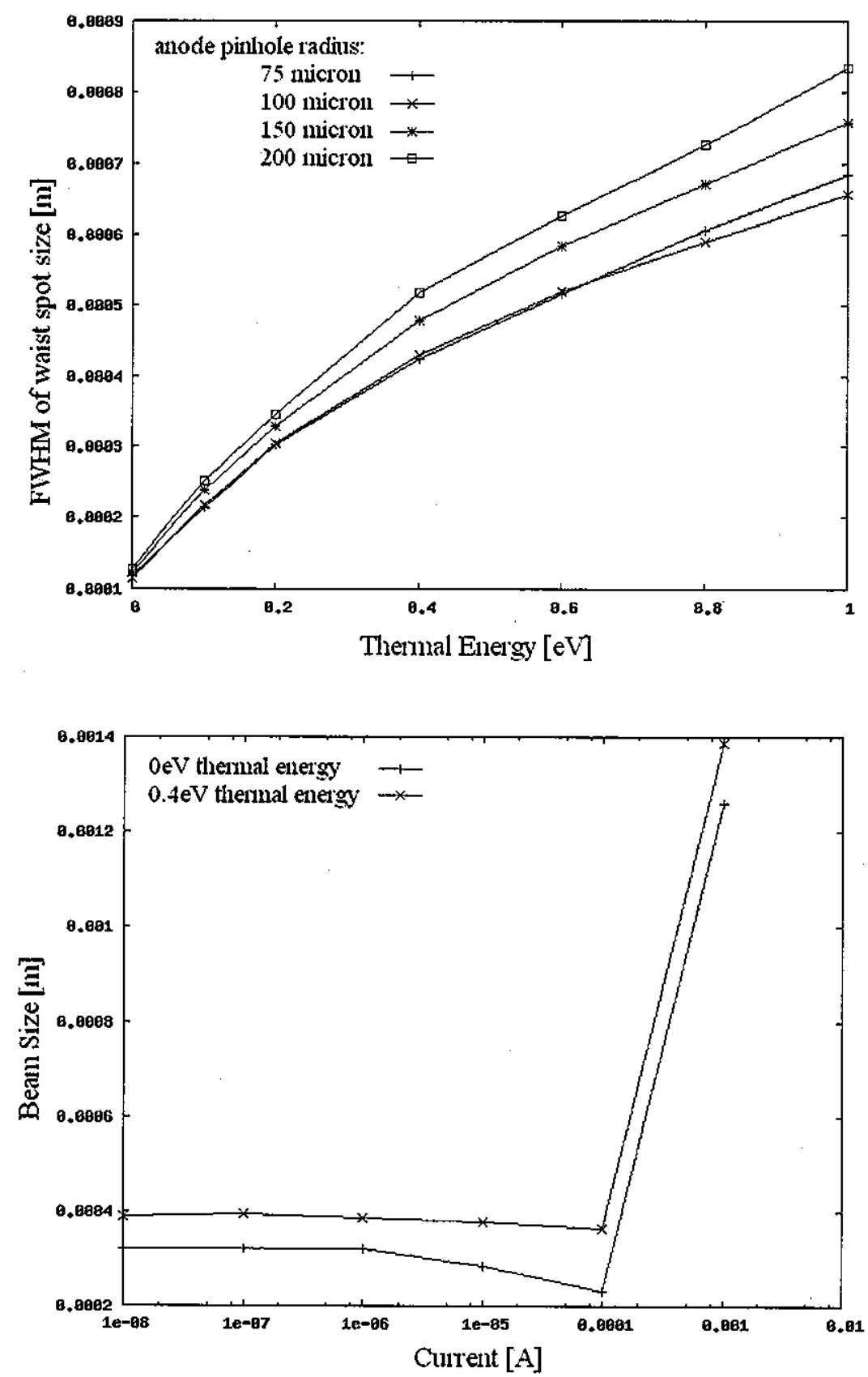

Figure 7.3: Aberration and space charge effects on beam spot size on screen.

The upper limit of the aperture and current is determined by a choice leading to a 
small change in the spot size. From the simulation above, an aperture radius of $100 \mu \mathrm{m}$ and a current of $10 \mu \mathrm{A}$ are chosen.

\subsection{Comparison of lenses}

The criterion of choosing the focusing lens is the growth rate in beam waist spot size with respect to thermal energy of the cathode. A large growth rate as a function of temperature will minimize the system errors. Results from simulation of different kinds of focusing lenses are shown in Figure 7.4 and Figure 7.5. All curves in both plots are simulated with same distance from anode to lens $(2 \mathrm{~cm})$ and from lens to screen $(25.5 \mathrm{~cm})$ for comparison. In both plots, the solenoid has a faster growth rate compared to other lenses. In Figure 7.4, the growth rate of a $0.5 \mathrm{~cm}$ aperture Einzel lens is comparable with a solenoid, but the nonlinear effect due to small aperture is significant, and this will result in large systematic error for spot size measurement. The simulation also shows that the closer the lens is to the anode and the further is the screen location, the better the resolution. 


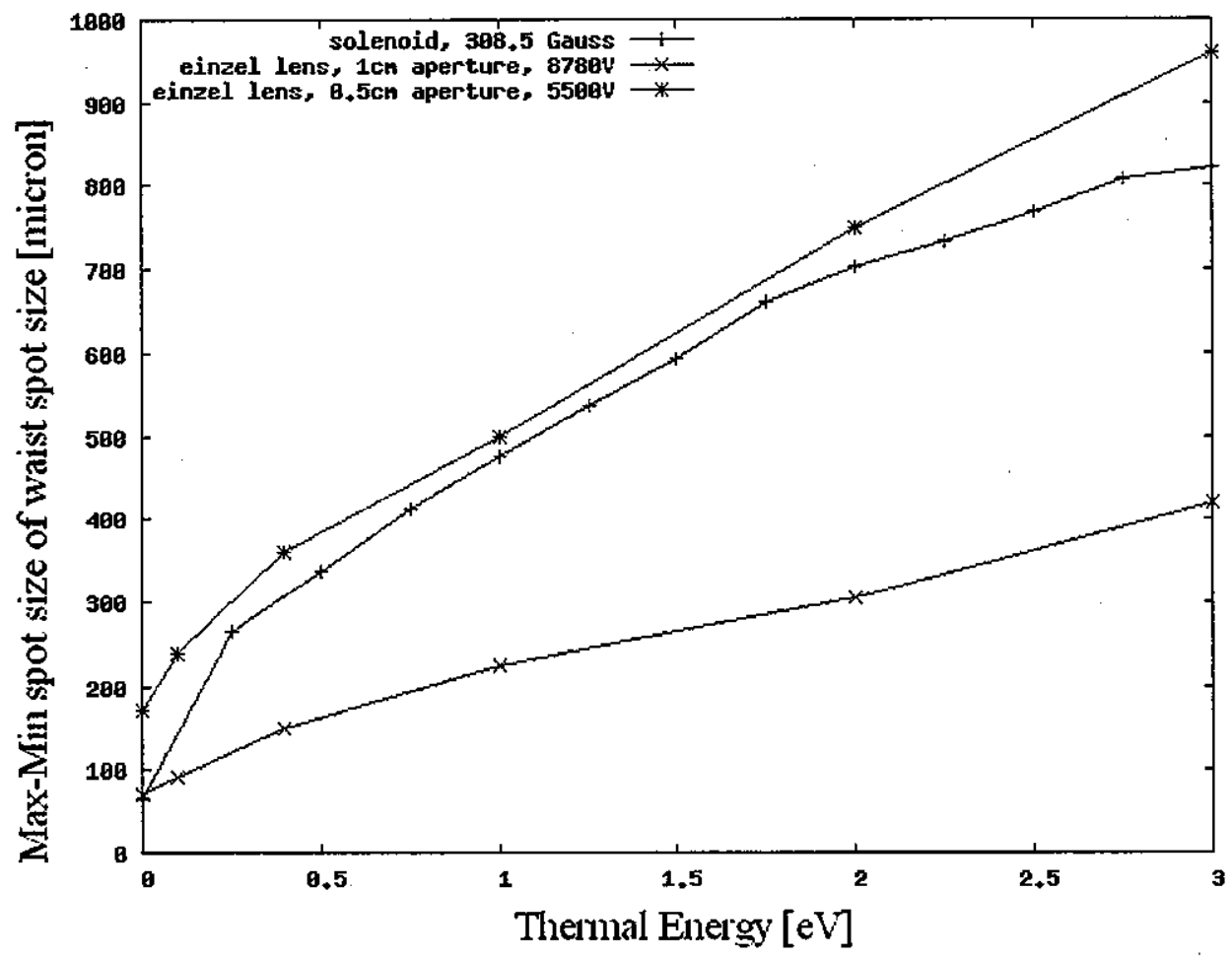

, Figure 7.4: Comparison of solenoid and two Einzel lenses. 


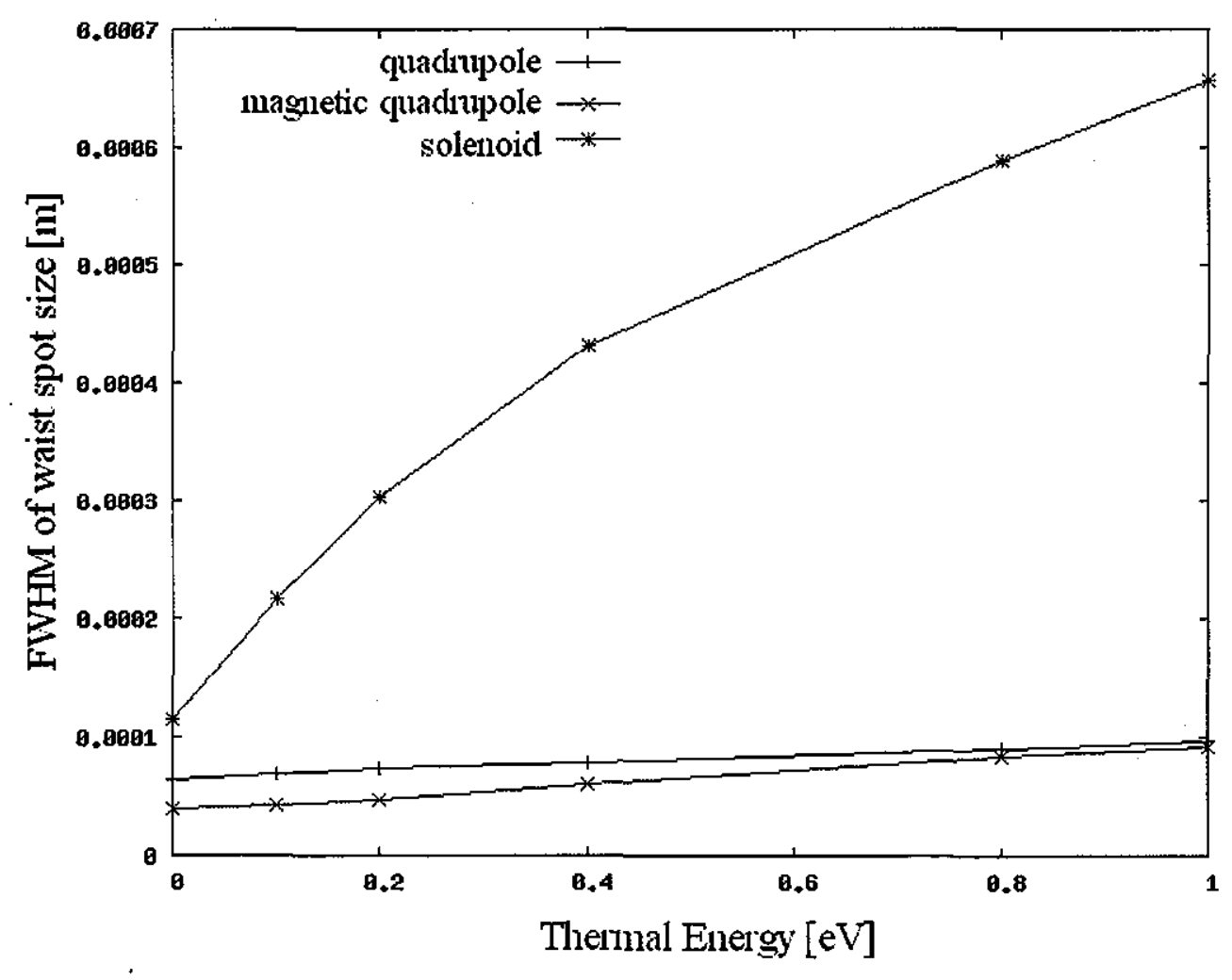

Figure 7.5: Comparison of solenoid and two quadrupoles.

From above simulation results, it can be concluded that solenoid is the final choice of focusing lens.

\subsection{Shielding}

All components in the measurement setup have low permeability. Shielding the solenoid is very important to prevent a magnetic force on the electrons during acceleration, which will reduce the resolution. Figure 7.6 shows the performance of a solenoid with and without shielding, and compares with hard edge field. 


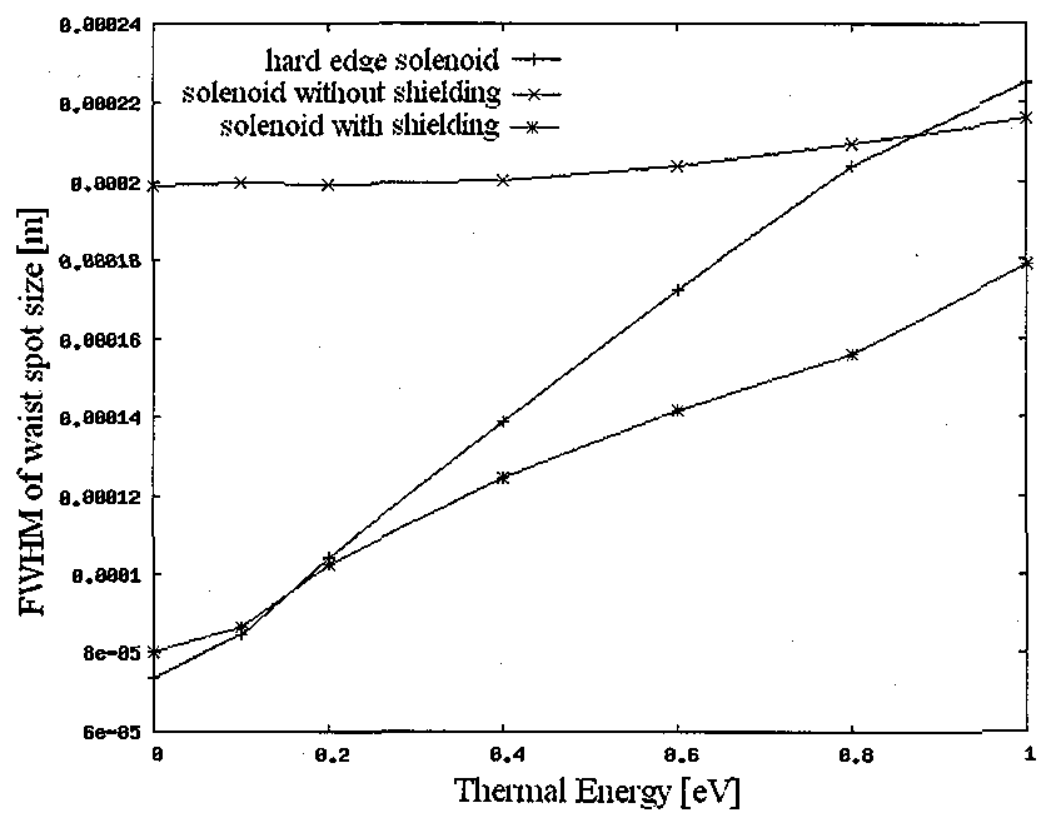

Figure 7.6: Solenoid with different shielding.

Shielding material of $1 \mathrm{~mm}$ thick with relative permeability $\mu_{r} \sim 20,000$ around the solenoid coil will decrease the magnetic field at the anode to $<1.5$ Gauss. Solenoid without shielding gives the worst resolution (green), while the resolution of solenoid with shielding of $\mu_{\mathrm{r}}=20,000$ (blue) is comparable with hard edge ideal case (red).

\subsection{Alignment}

The setup design is based on highly accurate alignment of the anode pinhole and the solenoid center axis. It is very important to have the secondary electron beam, after passing through the anode pinhole, symmetrically distributed around the solenoid center axis in the transverse direction. This avoids distortion of the waist spot 
on the screen.

During the alignment, a laser beam is passed through the system downstream of the electron path. Along the laser path, the anode pinhole and three additional $250 \mu \mathrm{m}$ diameter pinholes are used to conduct the alignment. Two are mounted on the center of the flanges at each end of the system. The third is located at the center of the start point of the solenoid field. With the laser beam passing all four pinholes, the offset of secondary electron beam away from the solenoid center axis is maintained to be less than $150 \mu \mathrm{m}$. 


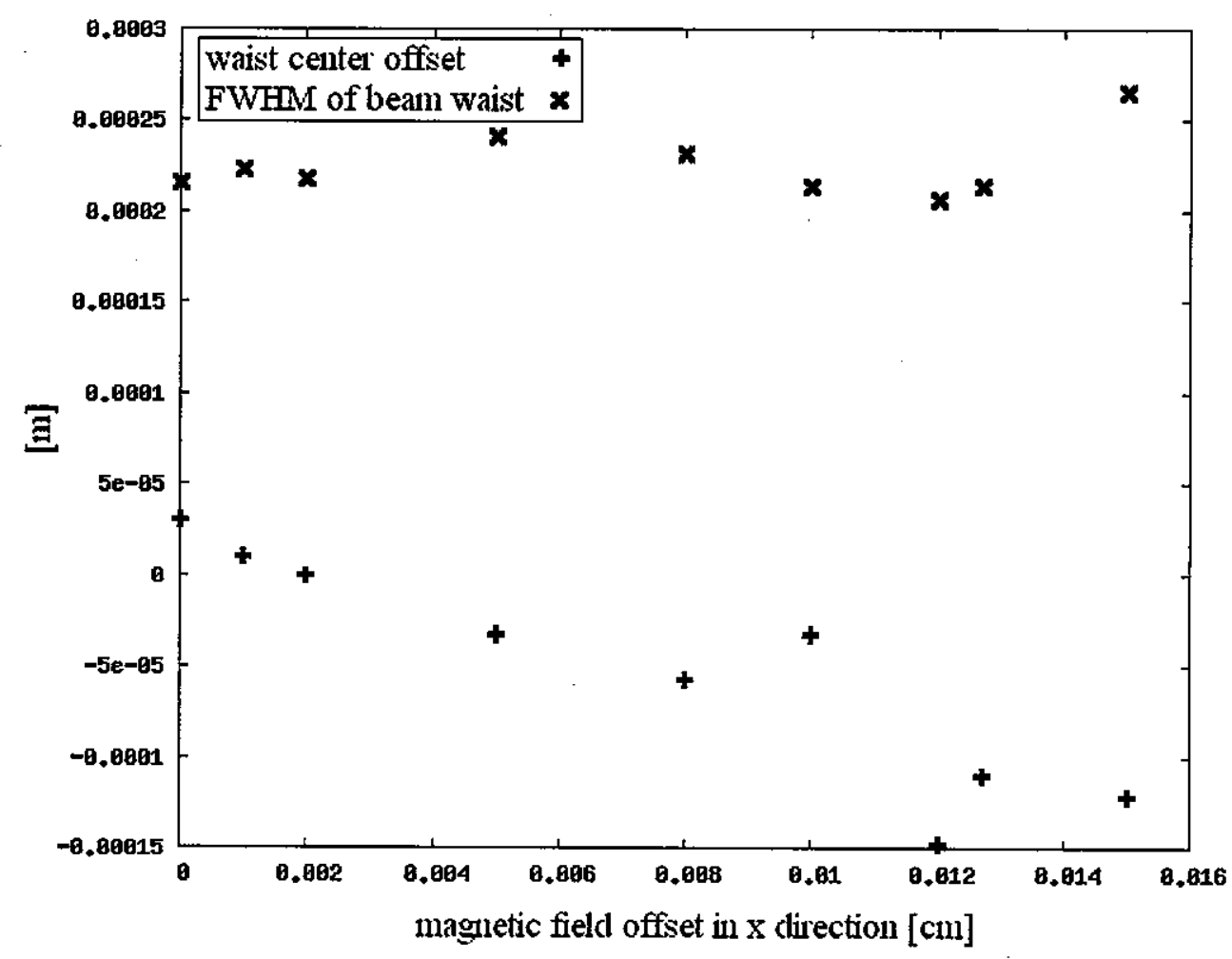

Figure 7.7: The offset magnetic field to the center of the electron beam and the waist.

FWHM difference according to the offset. Thermal energy of the beam is $0.1 \mathrm{eV}$.

This procedure also ensures that the solenoid axis is perpendicular to the anode surface, and the tilt angle can be controlled to better than $5 \mathrm{mrad}$.

Figure 7.7 shows the simulation of the beam waist FWHM change due to a different offsets of the electron beam for $0.1 \mathrm{eV}$ thermal energy. Thus the error caused by offset after alignment should be controlled within $15 \%$. A quadrupole lens may be added to the drift section for additional correction so as to lower the error even more. 


\subsection{Conclusion}

With the design of the solenoid lens based measurement system shown in Figure 7.1 and the calibrated simulation plot of FWHM waist spot size vs. thermal energy, a CCD camera with pixel size $\sim 5 \mu \mathrm{m}$ should be able to analyze the beam waist spot size down to $50 \mu \mathrm{m}$ diameter. Thus, we should be able to determine the thermal energy of diamond secondary emission to about $0.01 \mathrm{eV}$.

Careful alignment and shielding is very important in minimizing the systematic error.

The limit of thermal energy measurement from aberration of aperture of anode and space charge effect is $10 q_{\mu} \mathrm{m}$ in radius and $1 q_{\mathrm{A}} \mathrm{A}$ for secondary electrons respectively.

By changing the focusing strength of lens, the waist of electron beam will be imaged on screen at a specific location. For good resolution, lens should be as close to the anode as possible, and the screen should be relatively far away from the lens. 


\section{SUMMARY AND CONCLUSION}

The Diamond Amplified Photocathode (DAP) is a novel approach to generating electrons. By following the primary electron beam, which is generated by traditional electron sources, with an amplifier, the electron beam available to the eventual application is increased by 1 to 2 orders of magnitude in current.

Diamond has a very wide band gap of $5.47 \mathrm{eV}$ which allows for a good negative electron affinity with simple hydrogenation, diamond can hold more than $2000 \mathrm{MV} / \mathrm{m}$ field before breakdown. Diamond also provides the best rigidity among all materials. These two characters offer the capability of applying high voltage across very thin diamond film to achieve high SEY and desired emission phase. The diamond amplifier also is capable of handling a large heat load by conduction and sub-nanosecond pulse input.

The preparation of the diamond amplifier includes thinning and polishing, cleaning with acid etching, metallization, and hydrogenation.

The best mechanical polishing available can provide high purity single crystal diamond films with no less than $100 \mu \mathrm{m}$ thickness and $<15 \mathrm{~nm}$ Ra surface roughness. The ideal thickness for $700 \mathrm{MHz}$ beam is $\sim 30 \mu \mathrm{m}$, which requires further thinning with RIE or laser ablation. RIE can achieve atomic layer removal precision and roughness eventually, but the time consumption for this procedure is very significant. Laser 
ablation proved that with $<266 \mathrm{~nm}$ ps laser beam, the ablation process on the diamond can easily achieve removing a few microns per hour from the surface and $<100 \mathrm{~nm}$ roughness. For amplifier application, laser ablation is an adequate and efficient process to make ultra thin diamond wafers following mechanical polishing.

Hydrogenation will terminate the diamond surface with monolayer of hydrogen, and form NEA so that secondary electrons in the conduction band can escape into the vacuum. The method is using hydrogen cracker to strike hydrogen atoms onto the bare diamond surface to form $\mathrm{H}-\mathrm{C}$ bonds.

Two independent experiments were carried out to determine the transport of the electrons within the diamond and their emission at the surface. In transmission mode measurements, the diamond amplifier was coated with metal on both sides, so results simply depend only on the electron transport within the diamond. The SEY for this mode provides one secondary electron per $20 \mathrm{eV}$ energy, which gives the gain of more than 200 for $4.7 \mathrm{keV}$ (effective energy) primary electrons under $2 \mathrm{MV} / \mathrm{m}$. Laser detrapping can help the signal maintain the gain with $10 \mu$ s pulse and duty cycle of $1.67 \times 10^{-7}$. In emission mode measurements, in which the diamond is prepared as in the actual application, the SEY is $\sim 20$ for $700 \mathrm{eV}$ (effective energy) primary electrons under $1.21 \mathrm{MV} / \mathrm{m}$.

The electric field applied and the primary electron energy is limited by the experiment setup, but the results show good trend toward large gain under high field. 
Thermal emittance of the diamond secondary emission is critical for the beam application. A careful design is setup to measure with very fine precision and accuracy of $0.01 \mathrm{eV}$. 


\section{Reference:}

[1] R. Kuroda, Y. Hama, S. Kasahiwagi, H. Kawai, F. Nagasawa, M. Washio, H. Hayano, J. Urakawa, and X. J. Wang, European Particla Accelerator Conference, Paris, France, 2002.

[2] X. J. Wang, T. S. Rao, K. Batchelor, I. Ben-Zvi, and J. Fischer, Nuclear Instruments \& Methods in Physics Research Section a-Accelerators Spectrometers Detectors and Associated Equipment 356 (1995) 159.

[3] J. Grames, P. Adderley, J. Brittian, D. Charles, J. Clark, J. Hansknecht, M. Poelker, M. Stutzman, and M. Surles-Law, 2005 Particle Accelerator Conference, Knoxville, TN, 2005.

[4] L. M. Wang, 25th International Conference on Microelectronics, Belgrade, Serbia and Montenegro, 2006.

[5] F. Nava, C. Canali, C. Jacoboni, L. Reggiani, and S. F. Kozlov, Solid State Communications 33 (1980) 475.

[6] X. Chang, Ph.D Thesis, 2005.

[7] J. R. Olson, R. O. Pohl, J. W. Vandersande, A. Zoltan, T. R. Anthony, and W. F. Banholzer, Physical Review B 47 (1993) 14850.

[8] http://en.wikipedia.org/wiki/Thermal_conductivity

[9] J. Grimes, Master of Science Thesis, 2007.

[10] I. L. Krainsky and V. M. Asnin, Applied Physics Letters 72 (1998) 2574.

[11] K. R. Lykke, K. K. Murray, and W. C. Lineberger, Physical Review A 43 (1991) 6104.

[12] M. Scheer, R. C. Bilodeau, C. A. Brodie, and H. K. Haugen, Physical Review A 58 (1998) 2844.

[13] G. Haeffler, D. Hanstorp, I. Kiyan, A. E. Klinkmuller, U. Ljungblad, and D. J. Pegg, Physical Review A 53 (1996) 4127.

[14] D. M. Neumark, K. R. Lykke, T. Andersen, and W. C. Lineberger, Physical Review A 32 (1985) 1890.

[15] J. Slater, F. H. Read, S. E. Novick, and W. C. Lineberger, Physical Review A 17 (1978) 201.

[16] Z. Zhang, M. Wensell, and J. Bernholc, Physical Review B 51 (1995) 5291.

[17] J. Furthmuller, J. Hafner, and G. Kresse, Physical Review B 53 (1996) 7334.

[18] A. Laikhtman, A. Hoffman, R. Kalish, Y. Avigal, A. Breskin, R. Chechik, E. Shefer, and Y. Lifshitz, Applied Physics Letters 73 (1998) 1433.

[19] A. Shih, J. Yater, P. Pehrsson, J. Butler, C. Hor, and R. Abrams, Journal of Applied Physics 82 (1997) 1860.

[20] J. E. Yater and A. Shih, Journal of Applied Physics 87 (2000) 8103.

[21] J. Stöhr, NEXAFS spectroscopy, Springer, Berlin ; New York, 1996. 
[22] G. Christian, R. Stranger, S. Petrie, B. F. Yates, and C. C. Cummins, Chemistry-a European Journal13 (2007) 4264.

[23] X. Chang, I. Ben-Zvi, A. Burrill, J. Grimes, T. Rao, Z. Segalov, and J. Smedley, Particle Accelerator Conference, Albuquerque, NM, 2007.

[24] B. Ziaja, A. Szoke, D. van der Spoel, and J. Hajdu, Physical Review B 66 (2002).

[25] D. Dimitrov, R. Busby, J. Bruhwiler, J. Cary, I. Ben-Zvi, T. Rao, X. Chang, J. Smedley, and $\mathrm{Q}$. Wu, Particle Accelerator Conference, Albuquerque, NM, 2007.

[26] http://physics.nist.gov/PhysRefData/Star/Text/ESTAR.html

[27] W. Saslow, Bergstre.Tk, and M. L. Cohen, Physical Review Letters 16 (1966) 354.

[28] D. Dimitrov et al, unpublished results.

[29] S. Adachi, Handbook on Physical Properties of Semiconductors, Springer, New York, NY, 2004.

[30] L. Reggiani, S. Bosi, C. Canali, F. Nava, and S. F. Kozlov, Physical Review B 23 (1981) 3050.

[31] J. Isberg, A. Lindblom, A. Tajani, and D. Twitchen, Physica Status Soldi a-Applications and Materials Science 202 (2005) 2194.

[32] M. Nazare and A. Neves, Properties, Growth and Applications of Diamond, INSPEC, Inc., Stevenage, UK, 2000.

[33] Y. F. Zhang, Z. G. Zhou, J. W. Sun, and G. G. Chen, Physics Letters A 214 (1996) 76.

[34] A. S. Barnard and I. K. Snook, Journal of Chemical Physics 120 (2004) 3817.

[35] G. Bussetti, C. Goletti, P. Chiaradia, and T. Derry, Europhysics Letters 79 (2007).

[36] Z. E. Smith and S. Wagner, Physical Review B 32 (1985) 5510.

[37] G. Piantanida, A. Breskin, R. Chechik, O. Katz, A. Laikhtman, A. Hoffman, and C. Coluzza, Journal of Applied Physics 89 (2001) 8259.

[38] Q. Wu, I. Ben-Zvi, A. Burrill, X. Chang, D. Kayran, T. Rao, and J. Smedley, Particle Accelerator Conference, Albuquerque, NM, 2007. 